\title{
QUEEN'S
UNIVERSITY
BELFAST
}

\section{Photometric, polarimetric, and spectroscopic studies of the luminous, slow-decaying Type Ib SN 2012au}

Pandey, S. B., Kumar, A., Kumar, B., Anupama, G. C., Srivastav, S., Sahu, D. K., Vinko, J., Aryan, A., Pastorello, A., Benetti, S., Tomasella, L., Singh, A., Moskvitin, A. S., Sokolov, V. V., Gupta, R., Misra, K., Ochner, P., \& Valenti, S. (2021). Photometric, polarimetric, and spectroscopic studies of the luminous, slowdecaying Type Ib SN 2012au. Monthly Notices of the Royal Astronomical Society, 507(1), 1229-1253. https://doi.org/10.1093/mnras/stab1889

Published in:

Monthly Notices of the Royal Astronomical Society

Document Version:

Publisher's PDF, also known as Version of record

Queen's University Belfast - Research Portal:

Link to publication record in Queen's University Belfast Research Portal

Publisher rights

Copyright 2021 The Author(s) Published by Oxford University Press on behalf of Royal Astronomical Society.

This work is made available online in accordance with the publisher's policies. Please refer to any applicable terms of use of the publisher.

\section{General rights}

Copyright for the publications made accessible via the Queen's University Belfast Research Portal is retained by the author(s) and / or other copyright owners and it is a condition of accessing these publications that users recognise and abide by the legal requirements associated with these rights.

Take down policy

The Research Portal is Queen's institutional repository that provides access to Queen's research output. Every effort has been made to ensure that content in the Research Portal does not infringe any person's rights, or applicable UK laws. If you discover content in the Research Portal that you believe breaches copyright or violates any law, please contact openaccess@qub.ac.uk. 


\title{
Photometric, polarimetric, and spectroscopic studies of the luminous, slow-decaying Type Ib SN 2012au
}

S. B. Pandey, ${ }^{1 \star}$ Amit Kumar ${ }^{\circledR},{ }^{1,2 \star}$ Brajesh Kumar $^{\circledR},{ }^{1 \star}$ G. C. Anupama ${ }^{\circledR},{ }^{3}$ S. Srivastav, ${ }^{3,4}$ D. K. Sahu, ${ }^{3}$ J. Vinko, ${ }^{5,6,7}$ A. Aryan ${ }^{\circledR}, 1,8$ A. Pastorello, ${ }^{9}$ S. Benetti ${ }^{\circledR},{ }^{9}$ L. Tomasella ${ }^{\circledR}, 9$ Avinash Singh ${ }^{\circledR}, 1,10$ A. S. Moskvitin, ${ }^{11}$ V. V. Sokolov, ${ }^{11}$ R. Gupta, ${ }^{1,8}$ K. Misra ${ }^{\odot},{ }^{1}$ P. Ochner ${ }^{9}$ and S. Valenti ${ }^{12}$

${ }^{1}$ Aryabhatta Research Institute of Observational Sciences, Manora Peak, Nainital 263001, India

${ }^{2}$ School of Studies in Physics and Astrophysics, Pandit Ravishankar Shukla University, Chattisgarh 492010, India

${ }^{3}$ Indian Institute of Astrophysics, II Block, Koramangala, Bengaluru 560034, India

${ }^{4}$ Astrophysics Research Centre, School of Mathematics and Physics, Queen's University Belfast, Belfast BT7 1NN, UK

${ }^{5}$ Konkoly Observatory, Research Center for Astronomy and Earth Sciences, Konkoly Thege M. ut 15-17, Budapest 1121, Hungary

${ }^{6}$ Department of Optics and Quantum Electronics, University of Szeged, Dom ter 9, Szeged 6720, Hungary

${ }^{7}$ Department of Astronomy, University of Texas, Austin, TX 79712, USA

${ }^{8}$ Department of Physics, Deen Dayal Upadhyaya Gorakhpur University, Gorakhpur 273009, India

${ }^{9}$ INAF - Osservatorio Astronomico di Padova, Vicolo dell'Osservatorio 5, 35122 Padova, Italy

${ }^{10}$ Hiroshima Astrophysical Science Center, Hiroshima University, Higashi-Hiroshima, Hiroshima 739-8526, Japan

${ }^{11}$ Special Astrophysical Observatory of the Russian Academy of Sciences (SAO RAS), Nizhnij Arkhyz 369167, Russia

${ }^{12}$ Department of Physics, University of California, 1 Shields Avenue, Davis, CA 95616-5270, USA

Accepted 2021 June 28. Received 2021 June 23; in original form 2021 April 30

\begin{abstract}
Optical, near-infrared (NIR) photometric and spectroscopic studies, along with the optical imaging polarimetric results for SN 2012au, are presented in this article to constrain the nature of the progenitor and other properties. Well-calibrated multiband optical photometric data (from -0.2 to $+413 \mathrm{~d}$ since $B$-band maximum) were used to compute the bolometric light curve and to perform semi-analytical light-curve modelling using the MINIM code. A spin-down millisecond magnetar-powered model explains the observed photometric evolution of SN 2012au reasonably. Early-time imaging polarimetric follow-up observations $(-2$ to $+31 \mathrm{~d})$ and comparison with other similar cases indicate signatures of asphericity in the ejecta. Good spectral coverage of SN 2012au (from -5 to +391 d) allows us to trace the evolution of layers of SN ejecta in detail. SN 2012au exhibits higher line velocities in comparison with other SNe Ib. Late nebular phase spectra of SN 2012au indicate a Wolf-Rayet star as the possible progenitor for SN 2012au, with oxygen, He-core, and main-sequence masses of $\sim 1.62 \pm 0.15 \mathrm{M}_{\odot}, \sim 4-8 \mathrm{M}_{\odot}$, and $\sim 17-25 \mathrm{M}_{\odot}$, respectively. There is a clear absence of a first overtone of carbon monoxide (CO) features up to $+319 \mathrm{~d}$ in the $K$-band region of the NIR spectra. Overall analysis suggests that SN 2012au is one of the most luminous slow-decaying Type Ib $\mathrm{SNe}$, having comparatively higher ejecta mass $\left(\sim 4.7-8.3 \mathrm{M}_{\odot}\right)$ and kinetic energy $\left(\sim[4.8-5.4] \times 10^{51} \mathrm{erg}\right)$. Detailed modelling using MESA and the results obtained through STELLA and SNEC explosions also strongly support spin-down of a magnetar with mass of around $20 \mathrm{M}_{\odot}$ and metallicity $Z=0.04$ as a possible powering source of SN 2012au.
\end{abstract}

Key words: techniques: photometric-techniques: polarimetric-techniques: spectroscopic-supernovae: generalsupernovae: individual: SN 2012au - galaxies: individual: NGC 4790.

\section{INTRODUCTION}

Type Ib supernovae ( $\mathrm{SNe}$ ) are a subclass of stripped-envelope corecollapse $\mathrm{SNe}$ (SESNe), as the outer hydrogen $(\mathrm{H})$ envelopes of their progenitors are partially or completely removed because of the higher mass-loss rate before the explosion (Wheeler et al. 1987; Filippenko 1997; Gal-Yam 2017; Prentice \& Mazzali 2017; Shivvers et al. 2017; Modjaz, Gutiérrez \& Arcavi 2019). Weak signatures of $\mathrm{H}$ found in a few $\mathrm{SNe} \mathrm{Ib}$ may be attributed to the thin layer of $\mathrm{H}$ and its continuous stripping from the progenitor (Branch et al. 2006; Elmhamdi et al.

^E-mail: shashi@aries.res.in (SBP); amitkundu515@gmail.com (AK); brajesh.kumar@iiap.res.in (BK)
2006; Valenti et al. 2011; Hachinger et al. 2012). SNe Ib also serve as important events to probe deeper towards the proposed transition of H-poor SNe IIb to He-free SNe Ic (Filippenko, Matheson \& Ho 1993; Dessart et al. 2015; Yoon 2015). H-deficient Wolf-Rayet (WR) stars ( $20-25 \mathrm{M}_{\odot}$ ) are believed to be possible progenitors for Type IIb/Ib $\mathrm{SNe}$, as they have lost their $\mathrm{H}$ envelope partially/completely due to stellar winds (Heger et al. 2003; Georgy et al. 2009; Yoon 2015). On the other hand, low-mass progenitors $\left(\gtrsim 11 \mathrm{M}_{\odot}\right)$ in binary systems are also thought to be progenitors of these SESNe, where the primary star loses its $\mathrm{H}$ envelope through mass-transfer to a companion star (Podsiadlowski, Joss \& Hsu 1992; Nomoto, Iwamoto \& Suzuki 1995; Smartt 2009; Yoon, Woosley \& Langer 2010; Smith et al. 2011). Envelope stripping is crucial in aiding understanding of the evolution of massive stars, post-explosion interaction, and the properties of 
the resulting SNe (Yoon 2015; Gilkis et al. 2019). SNe Ib seem to have a higher/lower degree of envelope stripping and more/less massive progenitors in comparison with SNe IIb/Ic (Fang et al. 2019). More massive progenitors, in comparison with those for SNe of Type $\mathrm{IIb} / \mathrm{Ib} / \mathrm{Ic}$, are responsible for producing $\mathrm{H}$-deficient superluminous SNe (SLSNe I: Moriya, Sorokina \& Chevalier 2018; Gal-Yam 2019; Inserra 2019).

Probing pre-explosion images of nearby $\mathrm{SNe}$ sites is the best way to identify and determine physical properties like masses of progenitor stars (Gal-Yam et al. 2007; Van Dyk 2017). So far, because of the limitation of achievable spatial resolution, only a handful of SESNe are known, including iPTF13bvn (Ib: Cao et al. 2013; Folatelli, Van Dyk \& Kuncarayakti 2016), SN 2017ein (Ic: Kilpatrick et al. 2018; Van Dyk et al. 2018; Xiang et al. 2019), and the recent SN 2019yvr (Ib: Kilpatrick et al. 2021). Using pre-explosion images, a binary system for iPTF13bvn (Fremling et al. 2014; Kuncarayakti et al. 2015; Folatelli et al. 2016) and a single WR star for SN 2019yvr were found as potential progenitor candidates (Kilpatrick et al. 2021). However, since the site hosting SN 2017ein is very crowded, possibilities of the progenitor being a WR star, a binary system with a high-mass candidate, and an unresolved young compact star cluster exist together (Xiang et al. 2019).

The most acceptable explosion mechanism of such SESNe is radioactive decay of ${ }^{56} \mathrm{Ni}(\mathrm{RD})$; however, the light curves of some SESNe (e.g. Type Ib/c SN 2005bf: Maeda et al. 2007; Type Ic SN 2019cad: Gutiérrez et al. 2021; and many SLSNe I: Inserra et al. 2013; Nicholl, Guillochon \& Berger 2017; Kumar et al. 2021) are explained by the existence of a spin-down millisecond magnetar (MAG) as a powering source (Maeda et al. 2007; Kasen \& Bildsten 2010; Woosley 2010). Near-peak photometric data are important in performing analytical light-curve modelling, which helps in obtaining the power mechanism and estimating various physical parameters, such as the ejecta mass $\left(M_{\mathrm{ej}}\right)$, the kinetic energy of the explosion $\left(E_{\mathrm{K}}\right)$, and the ${ }^{56} \mathrm{Ni}$ mass (Chatzopoulos et al. 2012; Wheeler, Johnson \& Clocchiatti 2015). By contrast, late-phase spectral observations help in investigating the nature of the explosion mechanism/progenitor and in constraining the composition of the inner layers of the expanding ejecta, which allows us to estimate the amount of oxygen mass $\left(M_{\mathrm{O}}\right)$, He-core mass $\left(M_{\mathrm{He}}\right)$, and zeroage main-sequence mass (M $M_{\text {ZAMS }}$ ); see Uomoto (1986), Thielemann, Nomoto \& Hashimoto (1996), and Fang et al. (2019).

More useful information about such $\mathrm{SNe}$ can be probed using near-infrared (NIR) spectroscopy, which is important to understand molecular emission features not observed in the optical or UV regions (e.g. the carbon monoxide (CO) features in the $K$-band region) and is crucial to investigate dust formation (Morgan \& Edmunds 2003). Due to the large number of collisionally excitable energy levels of $\mathrm{CO}$ emission, it acts as a coolant and can shed light on the dust production rate in SN ejecta (Gearhart, Wheeler \& Swartz 1999; Morgan \& Edmunds 2003; Liljegren, Jerkstrand \& Grumer 2020). The NIR spectra of SNe Ib exhibit strong features of He I $\lambda 10800$ and $\mathrm{C}$ I, which give an idea about the extent of envelope stripping in these objects. He I features are found to be weaker/absent in SNe Ic (e.g. SN 2007gr: Hunter et al. 2009); however, in addition to this, broadline SNe Ic exhibit weak signatures of C I (SN 2020bvc: Rho et al. 2021), which indicate large envelope mass stripping in such events. Additionally, SNe Ib present stronger He I $\lambda 20580$ in comparison with that displayed in the NIR spectra of SNe Ic.

Asphericity is another generic property of SESNe, as half of these well-known events exhibit aspherical features owing to various possible effects like dust and the clumpy nature of ejecta (Leonard et al. 2006; Maeda et al. 2008; Wang \& Wheeler 2008; Taubenberger et al. 2009; Tanaka 2017). The level of asphericity in the ejecta could be estimated using the observed degree of polarization (Shapiro \& Sutherland 1982; McCall 1984; Hoflich 1991). The asphericity of the explosion can also be established independently by investigating the emission-line profiles (e.g. [O I] and [Ca II]) in optical nebular spectra, as suggested by Mazzali et al. (2001, 2005), Maeda et al. (2002, 2006), and Taubenberger et al. (2009). Among SESNe, observational signatures indicate a higher level of asphericity in $\mathrm{SNe}$ connected to gamma-ray bursts (GRB-SNe) than normal ones (Maeda et al. 2008). Therefore, understanding explosion/ejecta geometry is vital in probing the nature of possible progenitor and explosion mechanisms for these energetic events.

In the literature, based on early photometric and spectroscopic optical studies up to $\sim+140$ d, Takaki et al. (2013) claimed SN 2012au had properties similar to hypernovae, whereas, based on the comparatively higher absolute magnitude of SN 2012au, Milisavljevic et al. (2013) also suggested it as a possible golden link between SLSNe I and low-luminosity counterparts. Based on radio and X- ray observations, Kamble et al. (2014) suggested a smooth circumstellar environment around SN 2012au. However, using a very late-time spectrum (after 6.2 years of explosion), Milisavljevic et al. (2018) did not observe any signature of CSMI in the very late-time spectrum (at $+2270 \mathrm{~d}$ ) of SN 2012au and claimed it as a pulsar wind nebula remnant. In general, SLSNe I are $\sim 2-3$ magnitudes brighter and have roughly three times broader light-curve peaks than classical SNe (Gal-Yam 2019; Inserra 2019). However, investigations of a larger photometric sample suggest that $\mathrm{SNe} \mathrm{Ib} / \mathrm{c}$, Ic-BL, and SLSNeI display a continuum in luminosity distribution (De Cia et al. 2018). The near-maximum spectra of SLSNe I have unique dominant O II features, but late-phase spectra exhibit a resemblance with $\mathrm{SNe} \mathrm{Ib} / \mathrm{c}$ (Pastorello et al. 2010; Quimby et al. 2018). For this reason, SLSNe I and SESNe are expected to share similar powering mechanisms, although with diverse physical parameters for the former, such as comparatively higher $M_{\mathrm{ej}}$ or re-shocking of the ejecta by a central engine (Nicholl et al. 2015). The observational features of SN 2012au further prompted re-investigation using new sets of optical imaging photometric/polarimetric and spectroscopic data and the modelling resources used in the present study.

The presentation of the analysis is structured as follows. Multiband optical light curves of SN 2012au are presented in Section 2. Section 3 discusses semi-analytic model fitting to the bolometric light curve of SN 2012au. A polarimetric study is presented in Section 4. In Section 5, we present the optical and NIR spectroscopic evolution and estimation of basic parameters of SN 2012au using the nebular phase spectrum and also compare the line velocities of SN 2012au with those of other SNe Ib. Spectral comparisons of SN 2012au with other SESNe are discussed in Section 6. The MESA modelling of the progenitor is presented in Section 7. We discuss our results in Section 8 and conclude in Section 9. Throughout the work, the phase is computed with reference to the $B$-band maximum light, and magnitudes are expressed using the Vega scale.

\section{LIGHT CURVES OF SN 2012 AU}

The photometric monitoring of SN 2012au (J2000: $\alpha=12^{\mathrm{h}} 54^{\mathrm{m}} 52^{\mathrm{s}} .18$ and $\delta=-10^{\circ} 14^{\prime} 50^{\prime \prime}$. 2 ) was carried out using several facilities, including the Sampurnanand Telescope (ST-1.04m ${ }^{1}$ ), Devasthal Fast

\footnotetext{
${ }^{1}$ https://aries.res.in/facilities/astronomical-telescopes/104m-telescope
} 


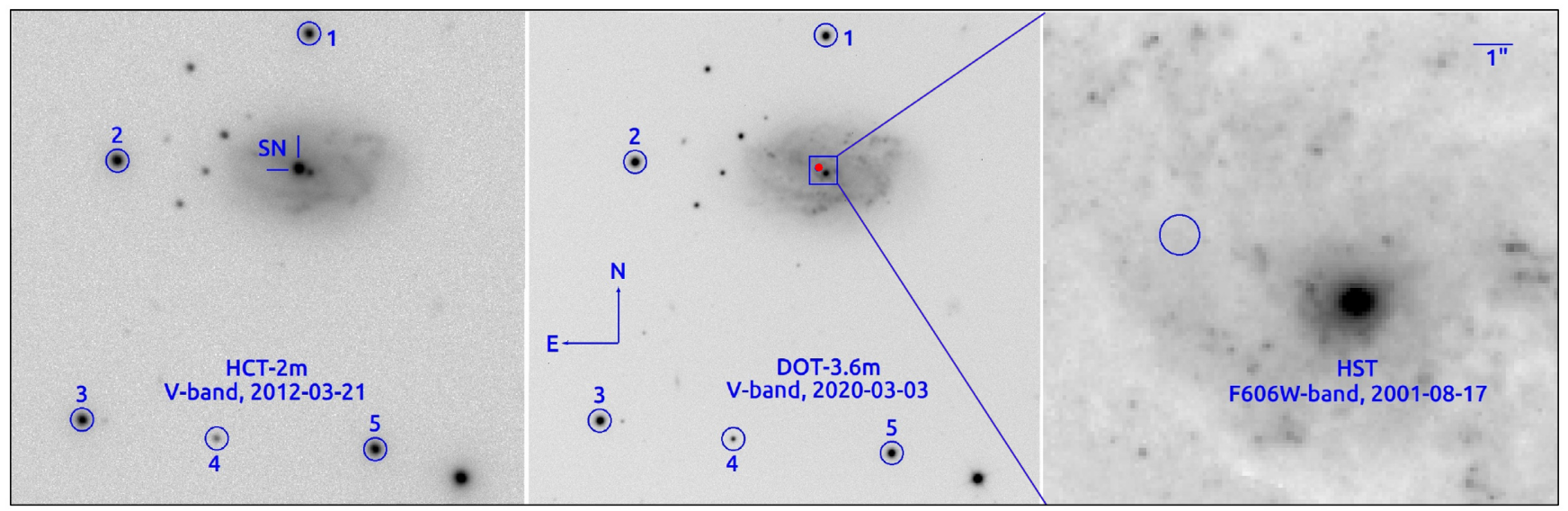

Figure 1. Identification chart of SN 2012au and local secondary stars (IDs 1-5). The $V$-band images obtained using HCT-2m and DOT-3.6m taken on 2012 March 21 and 2020 March 3 are shown in the left and middle panels, respectively. The field of view is roughly $3.5 \times 3.5$ arcmin ${ }^{2}$, and both images are astrometrically matched. The right panel shows a pre-explosion HST image observed on 2001 August 17, zoomed-in near the central region of the host galaxy NGC 4790. The SN 2012au location is marked in the left panel, and the same location is also indicated in the middle panel with a red dot after the SN has faded. The same location is shown with a circle (0.5-arcsec radius) in the pre-explosion HST image. North is up, and east is to the left.

Optical Telescope (DFOT-1.3 $\mathrm{m}^{2}$ ), Himalayan Chandra Telescope $\left(\right.$ HCT-2 $\mathrm{m}^{3}$ ), 2.2-m telescope on Calar Alto (CAHA-2.2m $\mathrm{m}^{4}$ ), and Big Telescope Alt-azimuth (BTA-6m ${ }^{5}$ ).

Our $U-, B-, V-, R$-, and $I$-band observations at 44 epochs spanned $\sim 414$ d. Data reduction was performed through standard procedures described in Kumar et al. (2021). Photometric calibrations were made using the secondary standards in the SN field, through independent observations of the Landolt standard fields with HCT$2 \mathrm{~m}$ and with the recently commissioned Devasthal Optical Telescope (DOT-3.6m ${ }^{6}$ ) operated by ARIES, Nainital, India (Kumar et al. 2018). The PG 0918 and SA 110 fields were observed with HCT2m on 2012 March 21 and 2012 April 21. Similarly, on 2020 March 3, observations of the PG 1323 field were performed with the $4 \mathrm{~K} \times 4 \mathrm{~K}$ CCD Imager mounted at DOT-3.6m (Pandey et al. 2018) to fine-tune the photometric calibrations. Using the Landolt standards, transformations to the standard system were derived by applying average colour terms and photometric zero-points. Average atmospheric extinction values in different bands for the Hanle and Devasthal sites were adopted from Stalin et al. (2008) and Mohan et al. (1999), respectively. Five secondary standard stars were used to calibrate the SN magnitudes. A finding chart indicating these stars (IDs 1-5) and the location of SN 2012au is shown in the left and middle panels of Fig. 1, and their magnitudes are listed in Table S1 of the supplementary material. A zoomed view of the SN 2012au field (about $0.7 \times 0.7 \operatorname{arcmin}^{2}$ ) as observed on 2001 August 17 in the F606W filter using the Hubble Space Telescope (HST) is also shown in the right panel of Fig. 1. The SN location is near the bright nucleus of the host galaxy. Consequently, the host contamination must be removed to infer the uncontaminated SN flux. In this study, template images of the field (without the supernova) were subtracted to obtain the final calibrated magnitudes. For this purpose, template images were acquired on 2014 February 26 with HCT-2m. All the images were first aligned with the GEOMAP and GEOTRAN tasks

\footnotetext{
${ }^{2}$ https://aries.res.in/facilities/astronomical-telescopes/13m-telescope

${ }^{3}$ https://www.iiap.res.in/iao/cycle.html

${ }^{4}$ http://www.caha.es/observing-mainmenu-148/telescopes-aamp-instrumen ts-mainmenu- 155

${ }^{5}$ https://www.sao.ru/tb/tcs/

${ }^{6} \mathrm{https}$ //aries.res.in/facilities/astronomical-telescopes/36m-telescope
}

in IRAF. Then, IRAF-based scripts were used to perform template subtraction and intensity matching of the template images to the frames with the SN. It is also worth mentioning that the photometry published by Takaki et al. (2013) was published without applying image subtraction. The marginal differences among photometry of $B V R I$ filters can be explained in terms of possible contamination by the host, based on our present careful analysis. Finally, aperture photometry was performed on the subtracted images, and the calibrated SN magnitudes are listed in Table S2 of the supplementary material.

The light curves of SN 2012au (in red) thus derived in the $U$, $B, V, R$, and $I$ bands are presented in Fig. 2. We correct the data of SN 2012au for a Galactic extinction of $E(B-V)=0.043 \mathrm{mag}$ (Schlafly \& Finkbeiner 2011) and a host galaxy extinction of $E(B-$ $V)=0.02 \pm 0.01 \mathrm{mag}$. The host galaxy extinction is adopted from Milisavljevic et al. (2013), calculated using the equivalent width of Na I D absorption; however, Takaki et al. (2013) assumed negligible host extinction. In the present analysis, the data have also been corrected for cosmological expansion. We derived absolute magnitudes from the extinction-corrected apparent magnitudes using

$M=m-5 \log \left(d_{\mathrm{L}} / 10 \mathrm{pc}\right)+2.5 \log (1+z)$.

Here, $M$ corresponds to the absolute magnitude, $m$ represents the apparent magnitude, and $d_{\mathrm{L}}$ is the luminosity distance (Hogg et al. 2002). We adopt a distance to the host NGC 4790 of $\approx 23.5 \pm 0.5$ Mpc, as given by Milisavljevic et al. (2013).

A third-order spline function was fitted around the approximate peak of the $B$-band light curve to measure the date of $B$-band maximum $\left(\mathrm{MJD}_{B \text {, peak }}=56006.4 \pm 0.5\right)$ and the corresponding absolute peak magnitude $\left(M_{B \text {, peak }}=-18.06 \pm 0.12 \mathrm{mag}\right)$. In the $R$ band, SN 2012au reached a peak brightness $\left(m_{R \text {, peak }}=13.18 \pm 0.02\right.$ mag) at $\mathrm{MJD}_{R \text {, peak }}=56009.2 \pm 0.5$, with an absolute peak magnitude $M_{R \text {, peak }}=-18.67 \pm 0.11 \mathrm{mag}$. The $M_{R \text {, peak }}$ of SN 2012au appears to be brighter in comparison with the mean $M_{R \text {, peak }}$ calculated for $\mathrm{SNe} \mathrm{Ib}$ and Ic, whereas it is lower in comparison with SNe Ic-BL (Drout et al. 2011, see the middle right panel of Fig. 2). It indicates that SN 2012au is one of the brightest SESNe. SN 2012au has been also observed in $J, H$, and $K$ bands using Son OF ISAAC (SOFI) on 


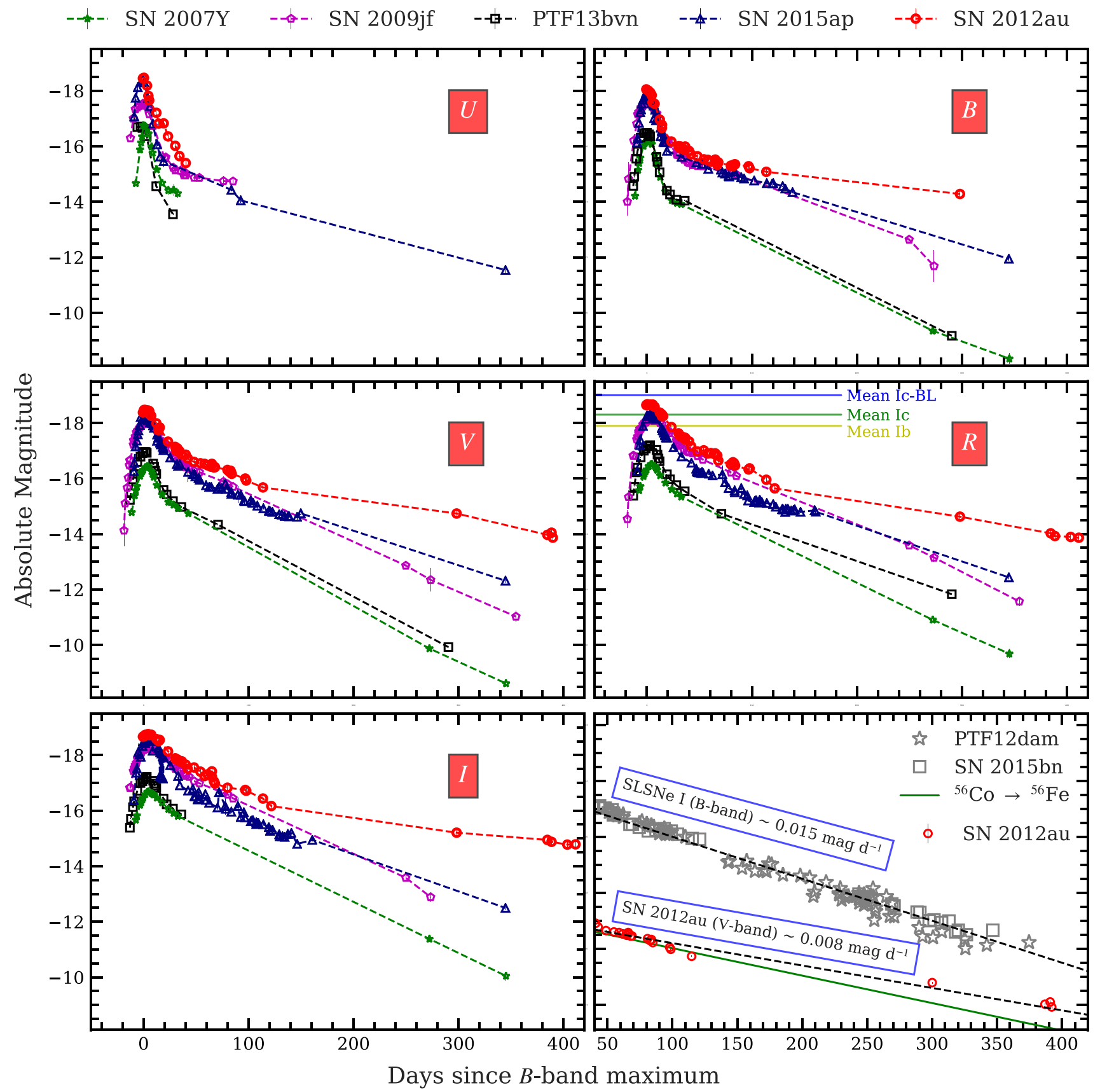

Figure 2. Multiband light-curve evolution of SN 2012au and other SNe Ib. The SN 2012au light curve peaks earlier in the bluer bands, as observed in other similar SNe (Taddia et al. 2018). SN 2012au is found to be one of the highly luminous SNe Ib, with a shallower post-peak decay rate in comparison with other $\mathrm{SNe} \mathrm{Ib}$. In the lower right panel, the $V$-band post-peak decay rate of SN 2012au is compared with $B$-band late-time light curves of two low-redshift slow-decaying SLSNe I (PTF12dam and SN 2015bn) and the ${ }^{56} \mathrm{Co} \rightarrow{ }^{56}$ fe decay curve.

the New Technology Telescope (NTT-3.58m $\left.{ }^{7}\right)$ at $56001.4(\sim-5 \mathrm{~d})$ and the apparent magnitudes are $13.40 \pm 0.06,13.33 \pm 0.10$, and $12.69 \pm 0.20 \mathrm{mag}$, respectively.

SN 2012au seems to have comparatively shallower post-peak decay rate (see Fig. 2) in all observed passbands in comparison with $\mathrm{SNe} \mathrm{Ib}$. From the peak to $+40 \mathrm{~d}$, the $U$-band light curve decays by $\sim 0.076 \mathrm{mag} \mathrm{d}^{-1}$, whereas the evolution is flatter in the redder

${ }^{7}$ https://www.eso.org/public/teles-instr/lasilla/ntt/ bands ( $\sim 0.029 \mathrm{mag} \mathrm{d}^{-1}$ in the $I$ band). At late phases ( +40 to $400 \mathrm{~d}$ ), the $B V R I$ light curves decay at $\sim 0.008 \mathrm{mag} \mathrm{d}^{-1}$, marginally slower than the ${ }^{56} \mathrm{Co} \rightarrow{ }^{56} \mathrm{Fe}$ decay rate $\left(0.0098 \mathrm{mag} \mathrm{d}^{-1}\right)$, see the bottom right panel of Fig. 2.

The $U, B, V, R$, and $I$ light curves of SN 2012au are compared with those of SN 2007Y (Stritzinger et al. 2009), SN 2009jf (Sahu et al. 2011; Valenti et al. 2011), iPTF13bvn (Folatelli et al. 2016; Fremling et al. 2016), and SN 2015ap (Gangopadhyay et al. 2020; Prentice et al. 2019). For comparison, the four SNe Ib are chosen as they have peak coverage and late-time photometric data $(z+250$ 
d) in at least five bands (UBVRI); see Fig. 2. SN 2007Y has data in the $u^{\prime} g^{\prime} B V r^{\prime} i^{\prime}$ bands, and these were transformed to the $U B V R I$ bands using the equations given by Jordi et al. (2006). The $\mathrm{MJD}_{B \text {, peak values }}$ for the four SNe Ib are estimated independently (see Table 1), and the light curves (in the unit of absolute magnitudes) are obtained using equation (1). SN $2012 \mathrm{au}$ exhibits the brightest $M_{B \text {, peak }}$ in comparison with other events. At later epochs $(\sim+40$ to $+400 \mathrm{~d})$, SN 2012 au light curves decay more slowly than the sampled SNe Ib, see Table 1. In the bottom right panel of Fig. 2, the $V$-band post-peak $(\sim+40$ to +400 d) decay of SN 2012au is compared with the $B$-band post-peak decay rates of two well-studied slow-decaying SLSNe I: PTF12dm (Nicholl et al. 2013) and SN 2015bn (Nicholl et al. 2016), which have latetime photometric observations. During +40 to +400 d, SN 2012au presents an even slower decay rate $\left(0.008 \mathrm{mag} \mathrm{d}^{-1}\right)$ than PTF12dam, SN 2015bn (0.015 mag d $\left.\mathrm{d}^{-1}\right)$, and a theoretical decay rate of ${ }^{56} \mathrm{Co}$ $\rightarrow{ }^{56} \mathrm{fe}$. In summary, SN 2012au is one of the brightest SESNe Ib with slower post-peak decay.

\subsection{Bolometric light curve of SN 2012au}

A quasi-bolometric (W1W2M2UBVRI) light curve of SN 2012au was generated using the PYTHON-based code SUPERBOL (Nicholl 2018a). Swift-UVOT data of SN 2012au in the W1W2M2 bands (from -4 to +32 d) were adopted from Milisavljevic et al. (2013). Most of the data points were available in the $R$ band, while data points at similar epochs in the other bands were obtained through interpolation or extrapolation, assuming a constant colour. To add the NIR flux contribution, we extrapolated the blackbody (BB) spectral energy distribution (SED) by integrating the observed UV-optical fluxes. The data points have also been dereddened using $E(B-V)=0.063$ mag, whereas the flux and wavelength values in individual bands were reported relative to the rest frame. In this way, we obtained the bolometric light curve (UV to NIR) from -0.2 to $+413 \mathrm{~d}$, with a peak luminosity of $\sim(6.56 \pm 0.70) \times 10^{42} \mathrm{erg} \mathrm{s}^{-1}$ at MJD $\approx$ $56005.8 \pm 1.0$, which is higher in comparison with the estimated mean peak luminosity for SNe IIb, Ib, and Ic, however lower than SNe Ic-BL (Lyman et al. 2016); see the upper panel of Fig. 3. The peak bolometric luminosity of SN 2012au is also higher than all SNe IIb, Ib, and Ic (except SN 2018cbz) reported by Prentice et al. (2019).

As discussed above, the late-time decay rate of the SN 2012au light-curve is even shallower than the theoretical decay curve of ${ }^{56} \mathrm{Co}$ $\rightarrow{ }^{56} \mathrm{Fe}$ (see Fig. 2). It is known that the luminosity of a magnetarpowered engine decays as $L \propto t^{-\alpha}$ with $\alpha=2$, a standard equation of a magnetic dipole (Kasen \& Bildsten 2010; Woosley 2010), see also Nicholl et al. (2018b). The late-time bolometric light curve of SN 2012au seems to trace the pattern well (in cyan colour, see the upper panel of Fig. 3) and indicate a magnetar origin.

We estimated the value of synthesized ${ }^{56} \mathrm{Ni}$ mass $\left(M_{\mathrm{Ni}}\right)$ of $\sim 0.27$ $\pm 0.07 \mathrm{M}_{\odot}$ using the relation between $M_{\text {peak }}$ and $M_{\mathrm{Ni}}$ given by Lyman et al. (2016). We also calculated $M_{\mathrm{Ni}} \sim 0.30 \mathrm{M}_{\odot}$ through a comparison with the synthetic light curve of total energy production, assuming a radioactive engine powered (in lime colour, see upper panel of Fig. 3) by the decay of the ${ }^{56} \mathrm{Ni} \rightarrow{ }^{56} \mathrm{Co} \rightarrow{ }^{56} \mathrm{Fe}$ decay chain (Nadyozhin 1994), closer to that estimated above. The estimated $M_{\mathrm{Ni}}$ for SN 2012 au in this study $\left(\sim 0.30 \mathrm{M}_{\odot}\right)$ is in good agreement with that suggested by Takaki et al. $\left(2013, \sim 0.3 \mathrm{M}_{\odot}\right)$.

Using the bolometric light curve of SN 2012au, we estimated $M_{\mathrm{ej}}, E_{k}$, and the characteristic time-scale $\left(T_{0}\right)$ using equations (1), (3), and (5), respectively (Wheeler et al. 2015). We adopted an electron-scattering opacity $(\kappa)=0.05 \mathrm{~cm}^{2} \mathrm{~g}^{-1}$ and opacity to gamma rays $\left(\kappa_{\gamma}\right)=0.03 \mathrm{~cm}^{2} \mathrm{~g}^{-1}$ for the present analysis. To calculate

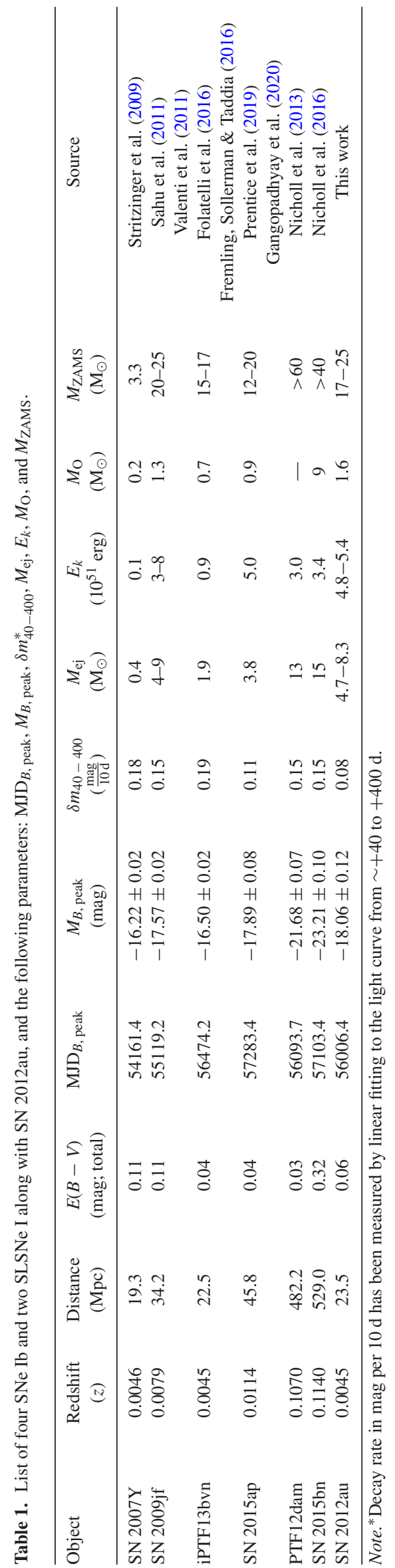



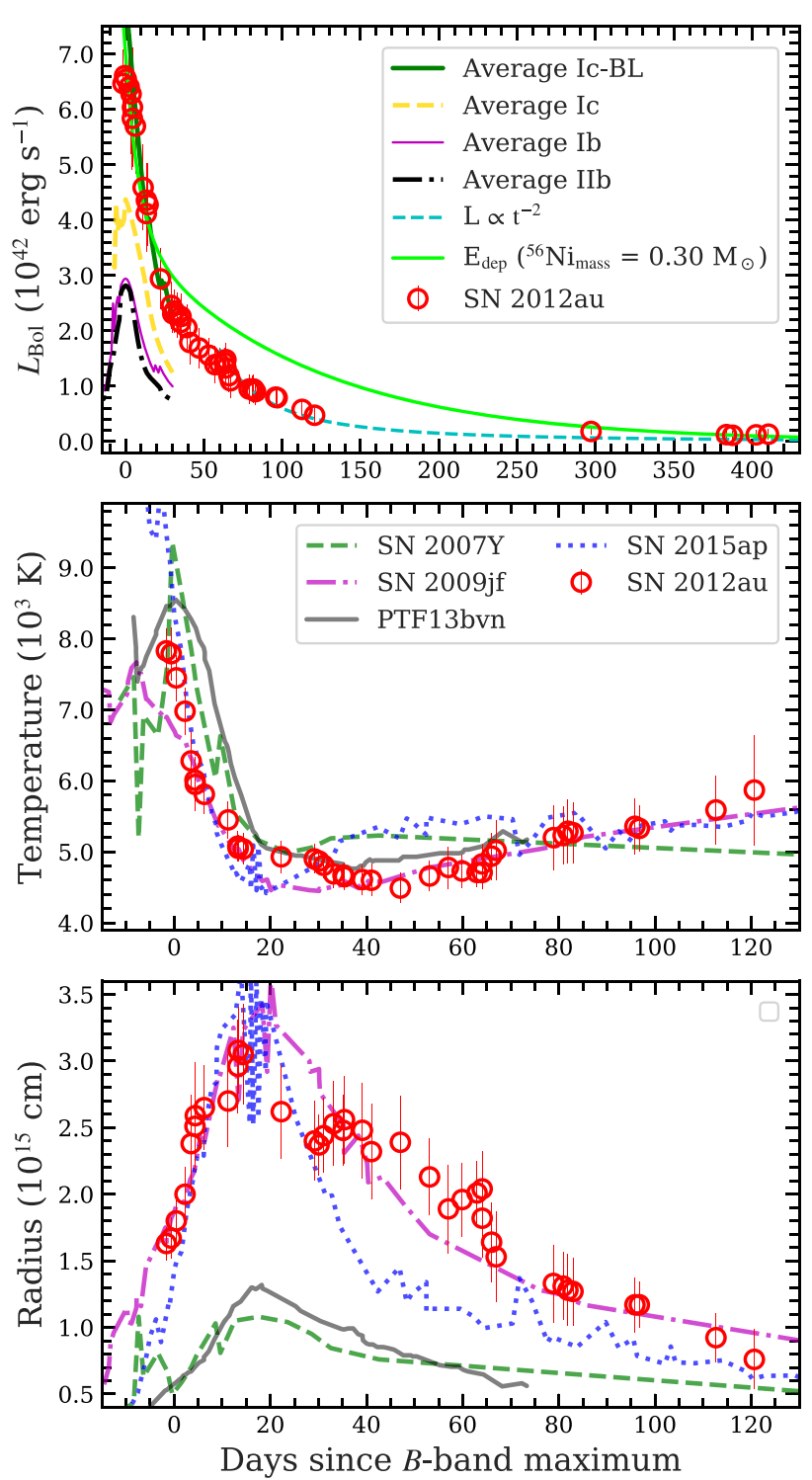

Figure 3. Top panel: bolometric light curve of SN 2012au, shown along with the average bolometric light curves of SNe IIb, Ib, Ic, and Ic-BL (Lyman et al. 2016). The full deposition rate of ${ }^{56} \mathrm{Ni} \rightarrow{ }^{56} \mathrm{Co} \rightarrow{ }^{56} \mathrm{Fe}$ decay chain, assuming $M_{\mathrm{Ni}}=0.30 \mathrm{M}_{\odot}$, is also shown in a lime colour. A standard equation of magnetic dipole ( $L \propto t^{-2}$ ) is also overplotted on the late-time bolometric light curve of SN 2012au (in cyan). Middle and bottom panels: $T_{\mathrm{BB}}$ and $R_{\mathrm{BB}}$ evolution of SN 2012au, obtained through a BB fit to the photometric data. $T_{\mathrm{BB}}$ and $R_{\mathrm{BB}}$ evolution of SN 2012au is close to that inferred for SN 2009jf, another bright SN Ib (Sahu et al. 2011).

the parameters discussed, a rise time $\left(t_{\mathrm{r}}\right)=16.3 \pm 1.0 \mathrm{~d}$ was estimated from the date of explosion (Milisavljevic et al. 2013) and the peak bolometric luminosity, while the photospheric velocity at peak luminosity ( $v_{\text {ph,peak }} \sim 12500 \pm 500 \mathrm{~km} \mathrm{~s}^{-1}$ ) was adopted from the spectral analysis (see Section 5.1.2). Using the above values of $t_{\mathrm{r}}$ and $v_{\mathrm{ph} \text {, peak }}$, we obtained $M_{\mathrm{ej}}, E_{k}$, and $T_{0}$ of $\sim 5.1 \pm 0.7 M_{\odot}$, $\sim 4.8 \pm 0.6 \times 10^{51} \mathrm{erg}$, and $\sim 66.0 \pm 9.4 \mathrm{~d}$, respectively. The values of $M_{\mathrm{ej}}$ and $E_{k}$ estimated for SN 2012au appear to be close to those found for SN 2009jf, whereas they are larger than those inferred for other SNe Ib listed in Table 1, indicating SN 2012au as one of the most highly energetic SN Ib. The $M_{\mathrm{ej}}$ value for SN 2012au is higher than those of SNe IIb, Ib, and Ic discussed by Wheeler et al.
(2015) and also higher than the mean values estimated for different samples of SNe IIb, Ib, Ic, and Ic-BL by Drout et al. (2011), Lyman et al. (2016), and Prentice et al. (2019), whereas it is nearly equal to those estimated for a sample of SNe Ic and SNe Ic-BL by Taddia et al. (2015). The evaluated $E_{k}$ of SN 2012au is higher than the mean values calculated for a sample of SNe Ib $\left(\sim 1.2 \times 10^{51} \mathrm{erg}\right)$ and Ic $\left(\sim 1.0 \times 10^{51} \mathrm{erg}\right)$, but lower in comparison with Ic-BL $\left(\sim 11 \times 10^{51}\right.$ erg) and engine-driven $\mathrm{SNe}\left(\sim 9 \times 10^{51} \mathrm{erg}\right)$, reported by Drout et al. (2011).

The evolution of $\mathrm{BB}$ temperature $\left(T_{\mathrm{BB}}\right)$ and the radius $\left(R_{\mathrm{BB}}\right)$ of SN 2012au are presented in the middle and lower panels of Fig. 3, respectively. The $T_{\mathrm{BB}}$ and $R_{\mathrm{BB}}$ parameters were calculated by modelling the SED at some epochs by fitting a BB function using the SUPERBOL code (Nicholl 2018a). The $T_{\mathrm{BB}}$ and $R_{\mathrm{BB}}$ evolution are shown only up to $+114 \mathrm{~d}$, because the BB approximations are poorer at later epochs. From peak to $\sim+12 \mathrm{~d}$, the $T_{\mathrm{BB}}$ of $\mathrm{SN} 2012 \mathrm{au}$ decreased from $\sim 8000$ to $4700 \mathrm{~K}$, whereas from $\sim+12$ to $+60 \mathrm{~d}$ it remains nearly constant at around $4600 \mathrm{~K}$. At later epochs (after $\sim+60 \mathrm{~d}), T_{\mathrm{BB}}$ increases slowly from $\sim 4600$ to $6000 \mathrm{~K}$. The $T_{\mathrm{BB}}$ evolution of SN 2012au is in good agreement with that of the SN Ib sample of Prentice et al. (2019). On the other hand, from the peak to $\sim+12 \mathrm{~d}, R_{\mathrm{BB}}$ of SN 2012au increases from $\sim 1.4 \times 10^{15}$ to $3.0 \times 10^{15}$ $\mathrm{cm}$, to decrease again after $+114 \mathrm{~d}$ to $\sim 0.5 \times 10^{15} \mathrm{~cm}$. The $T_{\mathrm{BB}}$ and $R_{\mathrm{BB}}$ evolution of SN 2012au is also compared with those estimated for SN 2007Y, SN 2009jf, iPTF13bvn, and SN 2015ap (see middle and lower panels of Fig. 3). The $T_{\mathrm{BB}}$ and $R_{\mathrm{BB}}$ values for iPTF13bvn and SN 2015ap are taken from Fremling et al. (2014) and Aryan et al. (2021), respectively, whereas they are calculated independently for SN 2007Y and SN 2009jf using the SUPERBOL code (Nicholl 2018a). Near the peak, SN 2012au exhibits lower temperature in comparison with SN 2007Y, iPTF13bvn, and SN 2015ap and higher than SN 2009jf, although after $\gtrsim+20 \mathrm{~d}$ all the SNe presented manifest a nearly similar trend of temperature evolution (see middle panel of Fig. 3). On the other hand, SN 2012au shares similar $R_{\mathrm{BB}}$ evolution to SN 2009jf and is also closer to SN 2015ap, although throughout the evolution SN 2012au presents higher values of $R_{\mathrm{BB}}$ in comparison with those estimated for SN 2007Y and iPTF13bvn (see lower panel of Fig. 3).

\subsubsection{Ejecta mass and kinetic energy}

The $M_{\mathrm{ej}}$ and $E_{K}$ values of SN 2012au estimated using the bolometric light-curve analysis are compared with those of classical $\mathrm{SNe} \mathrm{IIb}$ (23), Ib (33), Ic (33), Ic-BL (10), GRB-SNe (30), and SLSNe I (40) in Fig. 4. The parameters for SNe IIb, Ib, Ic, and Ic-BL were taken from Taddia et al. (2015), Wheeler et al. (2015), Lyman et al. (2016), Taddia et al. (2018), Prentice et al. (2019) and those of GRB-SNe from Cano (2017), while those of SLSNe I were from Nicholl et al. $(2015,2017)$. The $E_{K}$ values were not quoted by Nicholl et al. (2015, 2017) and Prentice et al. (2019), so we used equation (2) of Wheeler et al. (2015) to infer $E_{K}$ from $M_{\mathrm{ej}}$ and photospheric velocity $\left(v_{\mathrm{ph}}\right)$ Different types of SNe are plotted with different legends and colours, while SN 2012au is plotted with a red star.

From Fig. 4, it is clear than SN 2012au has the highest $M_{\mathrm{ej}}$ and $E_{K}$ values in comparison with most of the Type IIb (in green), Ib (in blue), and Ic (in orange). It also indicates that SN 2012au is one of the most energetic $\mathrm{SNe} \mathrm{Ib}$. SNe IIb, Ib, and Ic have a similar range of values for $E_{K}$ and $M_{\mathrm{ej}}$, while SNe Ic-BL seem to have comparatively high $E_{K}$ values, and GRB-SNe exhibit the highest $E_{K}$ values. The $E_{K}$ estimates for SLSNe I are similar to those for SNe IIb, Ib, and Ic, but they have a larger range of ejecta masses. In Fig. 4, ratios of 


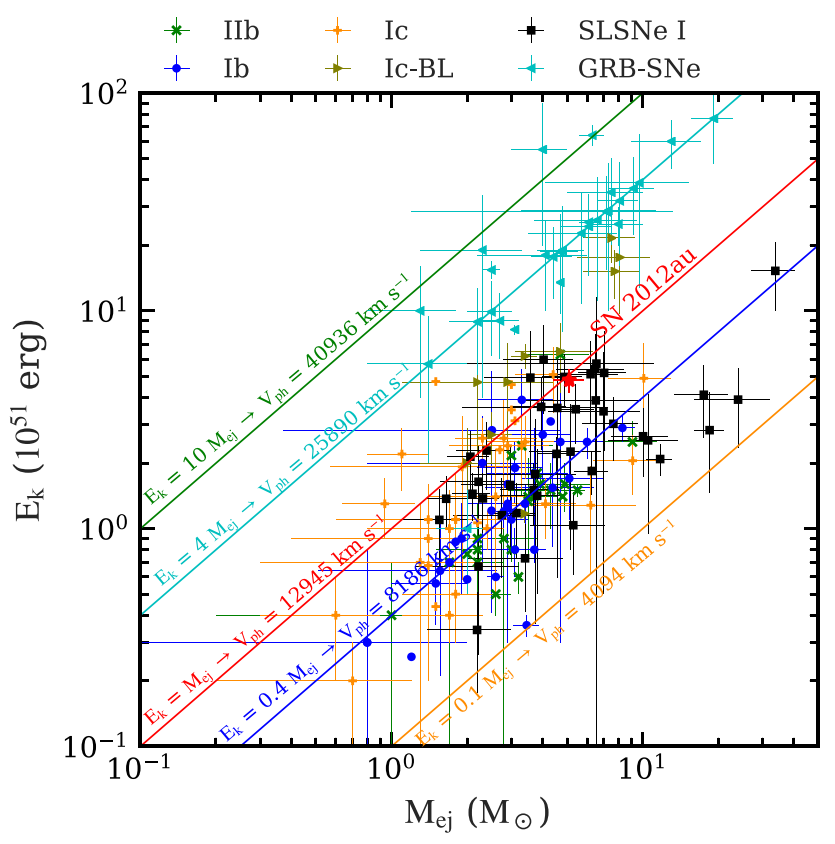

Figure 4. $M_{\mathrm{ej}}$ versus $E_{K}$ diagram for SN 2012au and a sample of SNe IIb, Ib, Ic, Ic-BL, GRB-SNe, and SLSNe I as discussed in Section 2.1.1. SN 2012au appears to have higher $E_{K}$ and $M_{\mathrm{ej}}$ in comparison with most SNe IIb (in green), Ib (in blue), and Ic (in orange), however lower than the values for Ic-BL and GRB-SNe.

$E_{K} / M_{\mathrm{ej}}$ (from 0.1 to 10 ) for corresponding $v_{\mathrm{ph}}$ values (from $\sim 4000$ to $41000 \mathrm{~km} \mathrm{~s}^{-1}$ ) are also shown in different colours. Most GRB-SNe converge to around $v_{\mathrm{ph}} \sim 25000 \mathrm{~km} \mathrm{~s}^{-1}$, whereas $\mathrm{SNe} \mathrm{IIb}, \mathrm{Ib}$, and Ic have a lower $v_{\text {ph }}$ range $\left(6000-13000 \mathrm{~km} \mathrm{~s}^{-1}\right)$. Most $\mathrm{SNe} \mathrm{Ilb}, \mathrm{Ib}$, and Ic lie below the $v_{\mathrm{ph}}$ curve of $\sim 13000 \mathrm{~km} \mathrm{~s}^{-1}$ (red line), which shows comparatively higher $v_{\mathrm{ph}}$ for SN 2012au.

\section{SEMI-ANALYTICAL LIGHT CURVE MODELLING USING MINIM}

The analytical model fitting to the bolometric light curve of SN 2012au was attempted using the MINIM code (Chatzopoulos et al. 2013). MINIM is a semi-analytical light curve modelling technique used to fit the light curves of SESNe. It uses the Price algorithm to look for the global minimum of the $\chi^{2}$ hypersurface within the allowed parameter volume. Corresponding to the minimum $\chi^{2}$, it provides a set of supernova explosion parameters including $M_{\mathrm{Ni}}, M_{\mathrm{ej}}$, ejecta opacity, etc. The working process of the MINIM code is described in detail by Chatzopoulos et al. (2013), see also Wheeler et al. (2017). Using MINIM, we attempted to fit the bolometric light curve of SN 2012au using various models like RD, MAG, constant density CSMI (CSMI0), wind-like CSMI (CSMI2), HYBRID (CSMI0 + RD), and HYBRIDW (CSMI2 + RD): see Fig. 5, briefly described below.

RD model: the radioactive decay model was originally developed by Arnett (1982) and it works on the assumptions discussed by Arnett (1982), Valenti et al. (2008), Chatzopoulos, Wheeler \& Vinko (2009), and Chatzopoulos et al. (2012, 2013). Under this model, $\gamma$ rays produced by the ${ }^{56} \mathrm{Ni} \rightarrow{ }^{56} \mathrm{Co} \rightarrow{ }^{56} \mathrm{fe}$ decay chain get trapped and thermalize the ejecta from centre to surface, powering the $\mathrm{SN}$ light curve. See equation (9) in Chatzopoulos et al. (2012) for the form of the resulting light curve based on $M_{\mathrm{Ni}}$, initial time $\left(t_{\text {ini }}\right)$, effective diffusion time-scale $\left(t_{\mathrm{d}}\right)$, and optical depth for $\gamma$-rays $\left(A_{\gamma}\right)$ as fitting parameters. The $M_{\mathrm{ej}}$ value is calculated using equation (10) of Chatzopoulos et al. (2012) assuming integration constant $(\beta)=$ $13.8, \kappa=0.05 \mathrm{~cm}^{2} \mathrm{~g}^{-1}$ (Drout et al. 2011; Milisavljevic et al. 2013), and $v_{\mathrm{ph} \text {, peak }}$ of $=12500 \pm 500 \mathrm{~km} \mathrm{~s}^{-1}$, which was adopted from the spectral analysis (see Section 5.1.2).

MAG model: under this model, a newly formed rapidly rotating millisecond magnetar (magnetized neutron star) is generated in the centre of the SN (Maeda et al. 2007; Kasen \& Bildsten 2010; Woosley 2010). In this scenario, conversion of magneto-rotational energy into radiation causes the newly-born 'magnetar' to spin down while simultaneously heating the $\mathrm{SN}$ ejecta. Refer to equation (13) in Chatzopoulos et al. (2012) for the form of the resulting light curve, which is mainly based on the magnetic dipole spindown formula. The fitting parameters of the MAG model are $t_{\text {ini }}$, progenitor radius $\left(R_{0}\right)$, magnetar rotational energy $\left(E_{\mathrm{p}}\right), t_{\mathrm{d}}$, magnetar spin-down time-scale $\left(t_{\mathrm{p}}\right)$, and ejecta expansion velocity $v_{\text {exp }}$. Similarly to the RD model, in the MAG model $M_{\text {ej }}$ is calculated using equation (10) of Chatzopoulos et al. (2012) assuming $\beta=$ $13.8, \kappa=0.05 \mathrm{~cm}^{2} \mathrm{~g}^{-1}$, and $v_{\text {ph, peak }}=12500 \pm 500 \mathrm{~km} \mathrm{~s}^{-1}$. The initial period of the magnetar $\left(P_{\mathrm{i}}\right)$ is given by $P_{\mathrm{i}}=10 \times$ $\left(2 \times 10^{50} \mathrm{erg} \mathrm{s}^{-1} / E_{\mathrm{p}}\right)^{0.5} \mathrm{~ms}$ and the magnetic field of the magnetar $(B)$ is calculated using $B=10^{14} \times\left(1.3 P_{10}^{2} / t_{\mathrm{p}, \mathrm{yr}}\right)^{0.5} \mathrm{G}$; here, $t_{\mathrm{p}, \mathrm{yr}}$ is the magnetar spin-down time-scale in years (Chatzopoulos et al. 2013).

CSMI model: as we know, the circumstellar environment is not always a vacuum and the ejecta may be surrounded by a thick CSM shell generated by mass loss from the massive star. SN ejecta can then collide and interact violently with the CSM. Eventually, shock heating can cause a very bright light curve, governed by the diffusion of thermalized photons to the photosphere, which is fixed at the outer radius of the shell (Chevalier \& Fransson 1994). Here, CSMI0 and CSMI2 models are designated to represent constant density and wind-like CSM, respectively. See equations (14) and (20) in Chatzopoulos et al. (2012) for the form of the resulting light curves. The fitting parameters in the case of the CSMI model are progenitor radius before the explosion $\left(R_{\mathrm{p}}\right), M_{\mathrm{ej}}$, CSM mass $\left(M_{\mathrm{csm}}\right)$, mass-loss rate $(\dot{M})$, and $v_{\text {exp }}$.

HYBRID model: nowadays, many of the complex light curves of peculiar SNe are not easy to explain using the single powering sources discussed above. Hence more than one powering source is proposed; these are termed as 'HYBRID' models (Chatzopoulos et al. 2012, 2013; Moriya et al. 2018; Chatzopoulos \& Tuminello 2019; Wang, Wang \& Dai 2019). In the present study, we also tried to regenerate the bolometric light curve of SN 2012au using combinations of CSMI and RD models. Refer to equation (21) in Chatzopoulos et al. (2012) for the form of the resulting light curve of the CSM + RD model. The fitting parameters used in the HYBRID model are the same as those discussed in the CSMI model plus $M_{\mathrm{Ni}}$. Details of all the models discussed above are given in Chatzopoulos et al. (2012, 2013).

All six models (RD, MAG, CSMI0, CSMI2, HYBRID, and HYBRIDW) discussed above could reproduce the bolometric lightcurve of SN 2012au with $\chi^{2} / \mathrm{DOF} \leqslant 1$ (where DOF stands for degrees of freedom); see Fig. 5. Here, we caution that $\chi^{2} / \mathrm{DOF}$ is used as an indicator for selecting the model parameters that fit the data best and not as a statistical probe for judging the significance of the models. In this situation, the best $\chi^{2}$ value alone cannot be used as a criterion to declare the most suitable model. Therefore, we considered various physical criteria to select different fitting parameters, as recently suggested in Kumar et al. (2021). The best-fitting parameters thus obtained from the MINIM modelling using the six models discussed are tabulated in Table 2. 

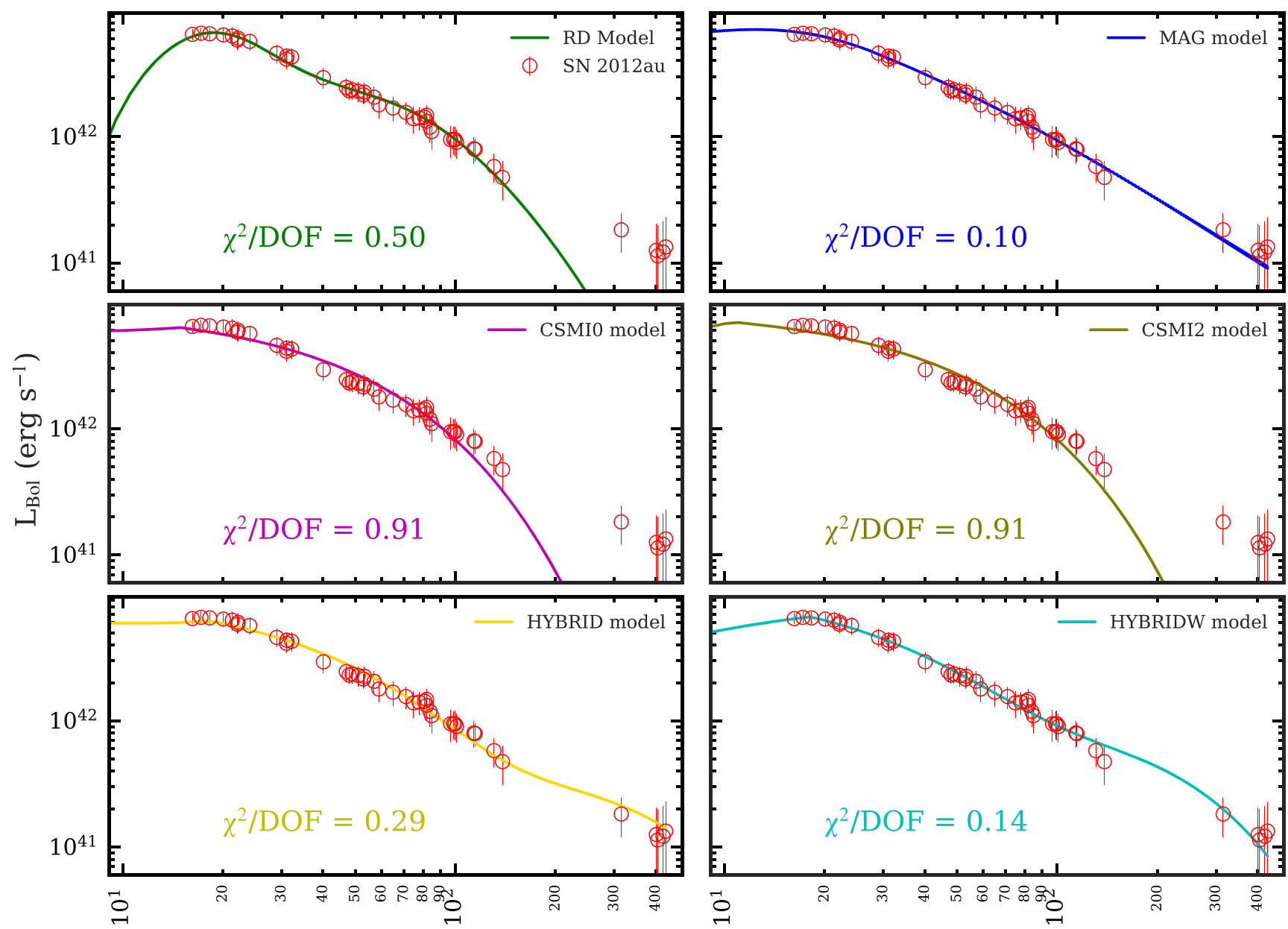

Days since explosion

Figure 5. Semi-analytic light curve models (RD, MAG, CSMI0, CSMI2, HYBRID, and HYBRIDW) fitted to the bolometric light curve of SN 2012au using the MINIM code (Chatzopoulos et al. 2013), shown in six different panels. The photometric data points of SN 2012au are shown by hollow red circles, whereas the six modelled light curves are colour-coded with the best-fitting modelled light curves.

Table 2. Best-fitting parameters for the RD, MAG, CSMI0, CSMI2, HYBRID, and HYBRIDW models using the MINIM code as described in Chatzopoulos et al. (2013).

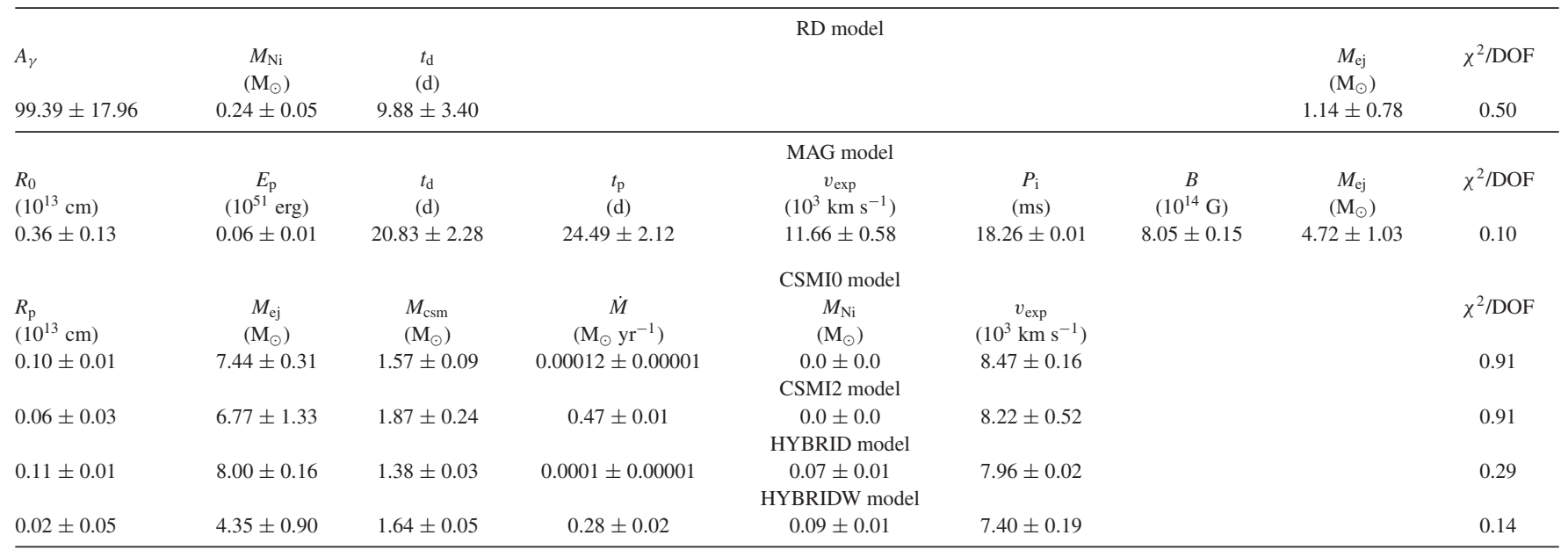


The RD model fits the light curve of SN 2012au nicely, but the $M_{\mathrm{ej}}$ is comparatively smaller than the value inferred from photometric analysis in Section 2.1. For this reason, the RD model is excluded from the possible powering mechanisms for SN 2012au. In the case of CSMI0, CSMI2, HYBRID, and HYBRIDW model fittings, $v_{\exp }$ is significantly lower than that obtained from the spectral analysis (see Section 5.1.1). In addition, in the case of CSMI2 and HYBRIDW models the $\dot{M}$ values are unreasonably high $\left(0.47\right.$ and $0.28 \mathrm{M}_{\odot}$ $\left.\mathrm{yr}^{-1}\right)$, so we exclude the possibility of dominance of the CSMI contribution to powering the SN 2012au light curve. The absence of narrow $\mathrm{H}$ Balmer lines in the late-time spectral observation (6.2 years after the explosion) also contradicts the CSMI as a dominant powering mechanism for SN 2012au (Milisavljevic et al. 2018). On the other hand, the MAG model fitted the SN 2012au very well (see upper-right panel of Fig. 5) and gives reasonable values of $M_{\text {ej }}\left(\approx 4.72 \pm 1.03 \mathrm{M}_{\odot}\right)$ and $v_{\text {exp }}\left(\sim 11.66 \pm 0.58 \times 10^{3} \mathrm{~km} \mathrm{~s}^{-1}\right)$, closer to those obtained from the photometric and spectral analysis. Hence, the MAG model has to be considered as the most suitable for SN 2012au. The value of $B\left(\sim 8 \times 10^{14} \mathrm{G}\right)$ computed in this study for SN 2012au is consistent with that estimated for another Type Ib SN 2005bf by Maeda et al. (2007). However, $P_{\mathrm{i}}(\sim 18 \mathrm{~ms})$ for SN 2012au is larger in comparison with SN 2005bf ( $\sim 10 \mathrm{~ms})$. In addition, Milisavljevic et al. (2018) also suggested a pulsar wind nebula remnant for SN 2012au, which is in favour of a central engine powering source.

\section{EARLY-PHASE IMAGING POLARIMETRY OF SN 2012 AU}

We also conducted imaging polarimetric observations of SN 2012au in the $R$ band at six epochs, using the ARIES Imaging Polarimeter (AIMPOL) mounted at the Cassegrain focus of ST-1.04m. This polarimeter consists of a half-wave plate (HWP) modulator and a Wollaston prism beam-splitter, and the images are captured with a CCD camera (Tektronix 1024 pixels $\times 1024$ pixels). Detailed information on the instrument can be found in Rautela et al. (2004). At each position of HWP position angle, multiple sets of frames were obtained, with individual exposure times being between 10 and 20 minutes. Images at each position of the HWP were combined to obtain a good signal-to-noise ratio. Table S3 in the supplementary material gives the complete log of the observations.

The ordinary and extraordinary fluxes were extracted by performing standard aperture photometry using IRAF. The Stokes parameters were estimated at different apertures (between 2 and 8 pixels), and finally $P$ (degree of polarization) and $\theta$ (polarization angle) were computed for the apertures that fitted best with minimum chi-square. The detailed procedures used to estimate $P$ and $\theta$ can be found in Ramaprakash et al. (1998), Rautela et al. (2004), and Kumar et al. $(2014,2016)$. The polarization angle was corrected for zeropoint polarization by observing various polarized standard stars from Schmidt, Elston \& Lupie (1992). It should be noted that the instrumental polarization of AIMPOL has generally been found to be $\sim 0.1$ per cent (Medhi et al. 2007; Pandey et al. 2009; Eswaraiah et al. 2012; Kumar et al. 2014, 2016, 2019; Srivastav et al. 2016, and references therein). In this study, the degree of polarization has been corrected by this offset value.

\subsection{Estimation of $\mathrm{SN}$ intrinsic polarization}

The directional extinction arising from dust grains located in the SN line of sight is a primary contaminating source that might influence the incoming SN polarization signal. To understand the intrinsic polarization properties of a $\mathrm{SN}$, the unwanted interstellar polarization (ISP) must be subtracted from the observed values. There is no direct formulation to estimate ISP precisely; however, it can be constrained by careful analysis of the nearby region.

To determine the Galactic ISP towards SN 2012au, we followed the procedures described in Kumar et al. (2014, 2016, 2019). We performed $R$-band polarimetric observations of nine isolated field stars within a $10^{\circ}$ radius of the $S N$ on 2013 January 20 . The reference stars were selected from the SIMBAD database from among those that do not show variability and spectral emission lines. The distance information for the nine stars was collected from Hipparcos parallax (van Leeuwen 2007) and Gaia data (DR2; Gaia Collaboration 2018). The observational details $\left(P_{R}, \theta_{R}\right.$, and distance) are listed in Table S4 of the supplementary material. The $P_{R}$ and $\theta_{R}$ values of these stars were converted to their respective Stokes parameters (i.e. $Q$ and $U$ ), and weighted linear fits were performed on the distance versus $Q$ and $U$. To estimate the Stokes parameters arising due to ISP, the slopes and intercepts were fitted at the most distant $(\sim 870 \mathrm{pc})$ field star HD 112325. The corresponding $P_{\text {ISP }}$ and $\theta_{\text {ISP values are computed }}$ as $<P_{\text {ISP }}>=0.23 \pm 0.01$ percent and $<\theta_{\text {ISP }}>=127.72^{\circ} \pm 1.09^{\circ}$, respectively.

According to Serkowski, Mathewson \& Ford (1975), the ISP and Milky Way (MW) reddening might be correlated with the mean and maximum polarization efficiency relation, i.e. $P_{\text {mean }}=5 \times E(B-$ $V)$ and $P_{\max }=9 \times E(B-V)$. The $E(B-V)$ towards SN 2012au is $0.063 \mathrm{mag}$ (see Section 2). If we assume that the dust along SN 2012au follows a mean polarization efficiency, it corresponds to $P_{\text {mean }}=0.32$ per cent. This is consistent with the $P_{\text {ISP }}$ value estimated with the nine field stars. We also used the three-dimensional map of dust reddening for the MW as described in Green et al. (2019). The map is based on Pan-STARRS and 2MASS photometry and Gaia DR2 parallaxes. The best fit towards SN 2012au provides $E(B-$ $V)=0.03$ mag and remains constant beyond a distance of $400 \mathrm{pc}$. Using this value, the polarization efficiency relations yield $P_{\text {mean }}$ and $P_{\max }$ as 0.15 and 0.27 percent, respectively. From the above exercises, it is inferred that the ISP upper limit is 0.32 percent and the ISP estimated from the field stars is considered for further analysis in this work.

At each epoch, the intrinsic polarization parameters of SN 2012au were estimated through a vectorial subtraction of the Stokes parameters $\left(Q_{\text {int }}=Q_{\mathrm{obs}}-Q_{\mathrm{ISP}}, U_{\mathrm{int}}=U_{\mathrm{obs}}-U_{\mathrm{ISP}}\right)$. The resulting intrinsic Stokes parameters were converted to $P_{\text {int }}$ and $\theta_{\text {int }}$ using the relation given in Kumar et al. (2019). The intrinsic (ISP-corrected) polarization parameters for SN 2012au are listed in Table S3 of the supplementary material.

\subsection{Polarimetric results and comparisons}

Polarimetric investigations of SESNe are important to constrain the level of asymmetry in the ejecta (Tanaka 2017). Polarization in $\mathrm{SNe}$ emerges due to incomplete cancellation of the directional components of electrical vectors. However, if the source is spherically symmetric, these vectors cancel each other and result in zero polarization (see Leonard \& Filippenko 2005; Wang \& Wheeler 2008). SNe Ib/c events exhibit a higher degree of polarization than SNe II, which indicates a departure from spherical symmetry (Chugai et al. 1992; Höflich, Khokhlov \& Wang 2001; Maund et al. 2007; Tanaka et al. 2008; Stevance 2019). The geometry of the circumstellar material may also contribute to the polarization evolution (Leonard et al. 2000; Hoffman et al. 2008; Mauerhan et al. 2014, 2017). Variation in the polarization parameters has been observed at different evolutionary phases of SESNe. Therefore, 


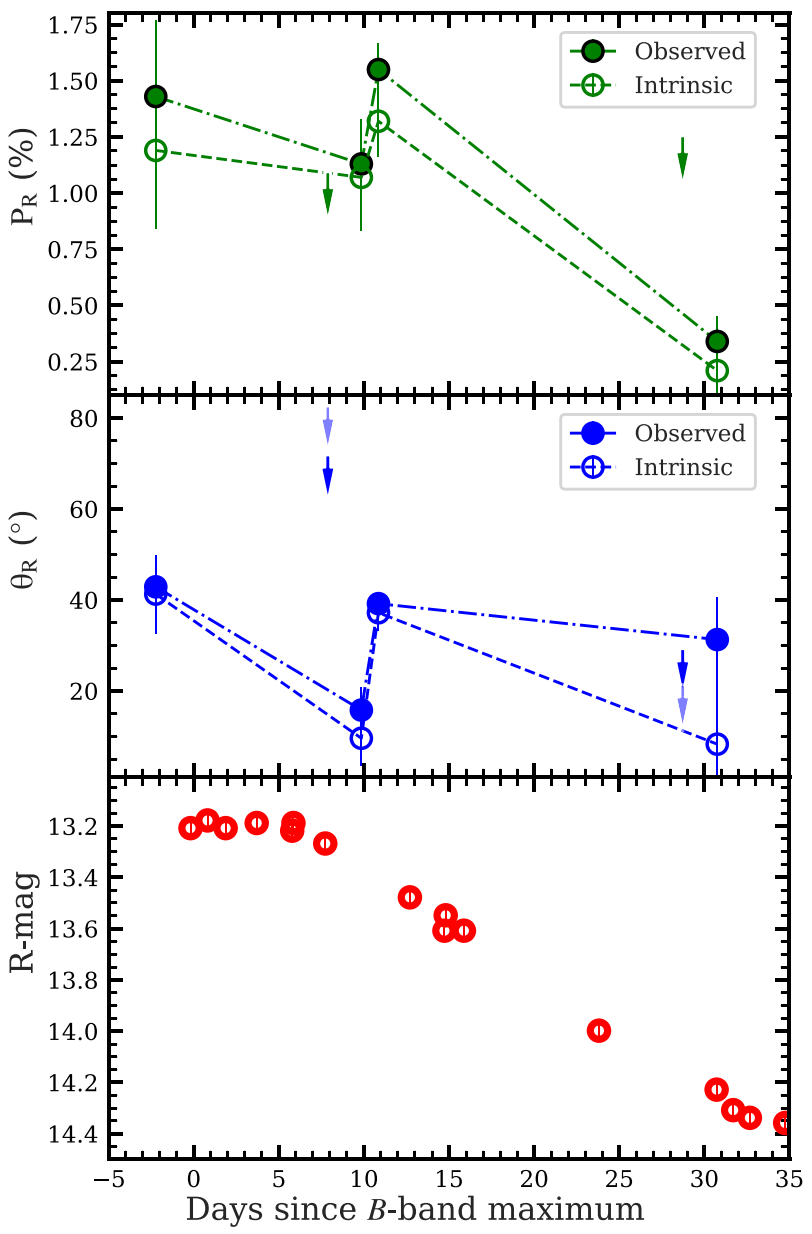

Figure 6. Temporal evolution of the polarization parameters of SN 2012au is shown. The degrees of polarization and the polarization angles are plotted in the upper and lower panels, respectively. The limiting values at two more epochs are shown with arrows. The lower panel shows the evolution of the apparent $R$-band magnitude during the same temporal bin.

multi-epoch polarimetric observations are crucial to understand the $\mathrm{SN}$ geometry and the underlying physics better. Imaging polarimetric and spectro-polarimetric techniques are generally used to estimate the linear polarization, which includes both continuum and line polarization. Imaging polarimetry provides a general picture of the explosion geometry or ejecta behaviour; on the other hand, spectrophotometric analysis and interpretations can be more conclusive in constraining polarization of various lines and the 3D geometry of the SN ejecta. In this work, we mainly focus on imaging polarimetric observations of SN 2012au and other similar SNe and their comparisons.

The imaging polarimetric evolution of SN 2012au in the $R$ band (covering He I $\lambda \lambda 5876,6678$, and $\lambda 7065$ ) is shown in Fig. 6 . The follow-up covers almost the whole photospheric phase of the light curve (between $\sim 14$ and $47 \mathrm{~d}$ from the explosion). During this period, the SN displays a maximum degree of polarization of 1.32 per cent (at $\sim 27 \mathrm{~d}$ ), fading to 0.13 percent (at $\sim 47 \mathrm{~d}$ ). A variation in the intrinsic polarization angle is also seen during this period, but it remains below $50^{\circ}$. Such evolution in the polarization parameters of SN 2012au indicates that the ejecta were aspherical and/or clumpy during the early phases. Using spectropolarimetric data at several

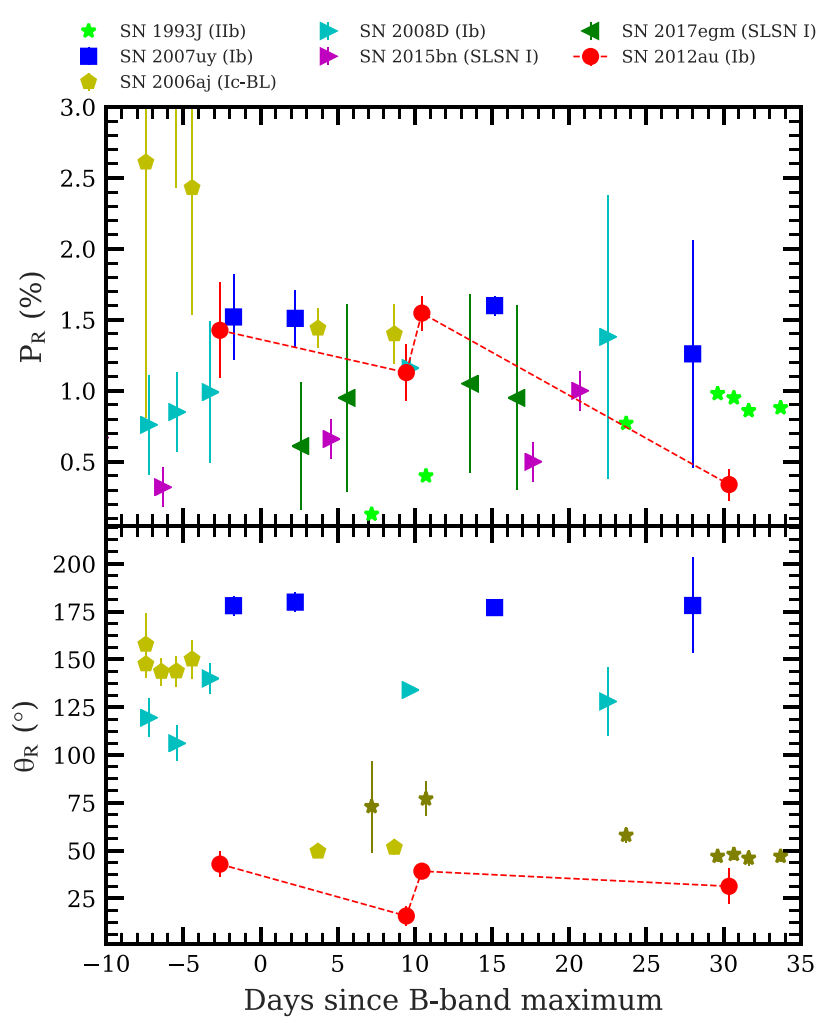

Figure 7. Evolution of the degrees of polarization of SN 2012au, compared with other SNe IIb, Ib, and SLSNe I from the literature.

epochs for this event, Hoffman et al. $(2014,2017)$ also noticed similar evolution in the continuum position angle.

The overall observational properties of SN 2012au show that it is a transitional event between SESNe and SLSNe I (cf. Milisavljevic et al. 2013; Takaki et al. 2013). Therefore, we also compared (see Fig. 7) the linear imaging polarization properties of SNe Ib (SN 2007uy and SN 2008D: Maund et al. 2009; Gorosabel et al. 2010), IIb (SN 1993J: Doroshenko, Efimov \& Shakhovskoi 1995), GRB-SN (SN 2006aj: Gorosabel et al. 2006), and SLSNe I (SN 2015bn: Leloudas et al. 2017; SN 2017egm: Maund et al. 2019) from the literature with those determined for SN 2012au. Here, we consider only events with multi-epoch observations. This analysis reveals that, among SNe Ib, SN 2008D and SN 2012au show a major variation in polarization parameters, whereas the SN 2007uy evolution is minimal. Similarly, SN 2015bn exhibits an increasing trend in the degree of polarization, while SN 2017egm remains below 1 per cent, without any significant change. Pre-maximum, SN 2006jf exhibits high values of liner polarization in comparison with all SNe presented; however, after the peak it shares polarization values closer to SN 2012au.

\subsubsection{Evolution of $\mathrm{E}_{\mathrm{k}} / \mathrm{M}_{e j}$ and $\mathrm{M}_{N i}$ with degree of polarization}

In this section, per cent $P$ versus $E_{k} / M_{\mathrm{ej}}$ and $M_{\mathrm{Ni}}$ of SN 2012au are compared with those of $\mathrm{SNe} \mathrm{IIb}, \mathrm{Ib}$, Ic, and GRB-SNe (see the upper and lower panels of Fig. 8, respectively) from the literature: SN 1993J (Doroshenko et al. 1995; Lyman et al. 2016), SN 1998bw (Patat et al. 2001), SN 2002ap (Wang et al. 2003; Mazzali et al. 2002; Pandey et al. 2003), SN 2003dh (Kawabata et al. 2003; Covino et al. 2003; Cano 2017), SN 2005bf(Maund et al. 2007), SN 2006aj 


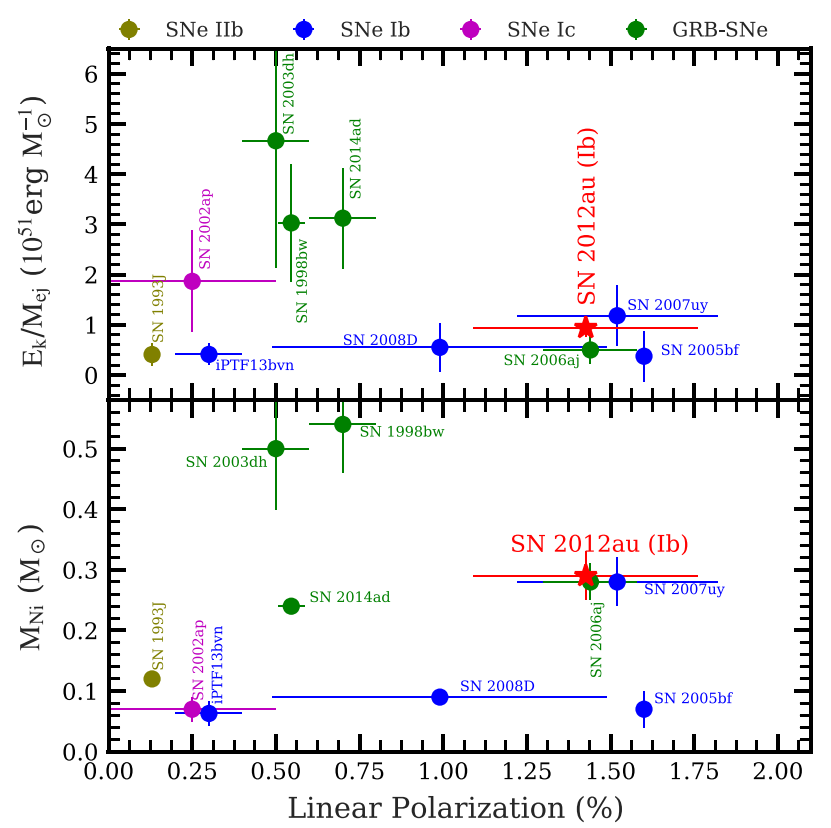

Figure 8. Top panel: linear polarization versus $E_{k} / M_{\mathrm{ej}}$ of $\mathrm{SN} 2012 \mathrm{au}$ compared with other SNe Ib, Ic, and Ic-BL in the literature. Bottom panel: linear polarization versus $M_{\mathrm{Ni}}$ of SN 2012au compared with the same sample.

(Gorosabel et al. 2006; Cano 2017), SN 2007uy (Gorosabel et al. 2010), SN 2008D (Gorosabel et al. 2010), iPTF13bvn (Reilly et al. 2016), and SN 2014ad (Stevance et al. 2017; Sahu et al. 2018). The polarimetric values for comparison were taken near the optical maximum $(\sim-10$ to $+10 \mathrm{~d}$ ). With the exception of SN 1998bw (from 4000-7000 ̊̊), SN 2002ap (from continuum), SN 2005bf ( 3000 $\AA$ ), iPTF13bvn (from continuum), and SN 2014ad ( $V$ band), for all other events $R$-band polarimetric values were adopted.

From Fig. 8, SN 2012au appears to have a near-peak per cent $P$ value closer to those of SN 2006aj and SN 2007uy. SN 1998bw and $\mathrm{SN} 2003 \mathrm{dh}(\mathrm{GRB}-\mathrm{SNe})$ present higher values of $E_{k} / M_{\mathrm{ej}}$ and $M_{\mathrm{Ni}}$, but exhibit lower per cent $P$ values in comparison with SN 2012au. Among SNe Ib, SN 2012au exhibits a higher per cent $P$ value in comparison with SN 2008D and iPTF13bvn (lowest values of per cent $P$ ), closer to SN 2007uy and lower than SN 2005bf. In our limited sample, SN 2005bf (Type Ib/c) exhibits the highest value of linear polarization with a very low value of $M_{\mathrm{Ni}}$, whereas SN 1993J shares the lowest value of per cent $P$. SLSNe I are nearly 2-3 mag brighter than normal SESNe, which corresponds to $M_{\mathrm{Ni}} \gtrsim 5 \mathrm{M}_{\odot}$ (Gal-Yam 2012). Therefore, due to comparatively high $M_{\mathrm{Ni}}$, SLSNe I will show a different class in Fig. 8, hence they are not included.

\section{SPECTROSCOPIC ANALYSIS}

We present spectroscopic studies of SN 2012au using unpublished spectra: 21 at optical and 2 at NIR wavelengths, spanning the range from -5 to $+391 \mathrm{~d}$. Spectra used here were obtained using a few telescopes during 2012-2013 (see Table S5 in the supplementary material for the spectroscopic observation $\log$ ) as part of the present work. Out of 21 optical spectra, 15 were obtained using HCT-2m, one using the Galileo-1.22m telescope ${ }^{7}$ (Asiago, Italy), one using CAHA- $2.2 \mathrm{~m}$, two using NTT- $3.58 \mathrm{~m}$, and two using the BTA-6m telescope. On the other hand, both the NIR spectra of SN 2012au (at -5 and $+21.8 \mathrm{~d}$ since maximum) were obtained using SOFI

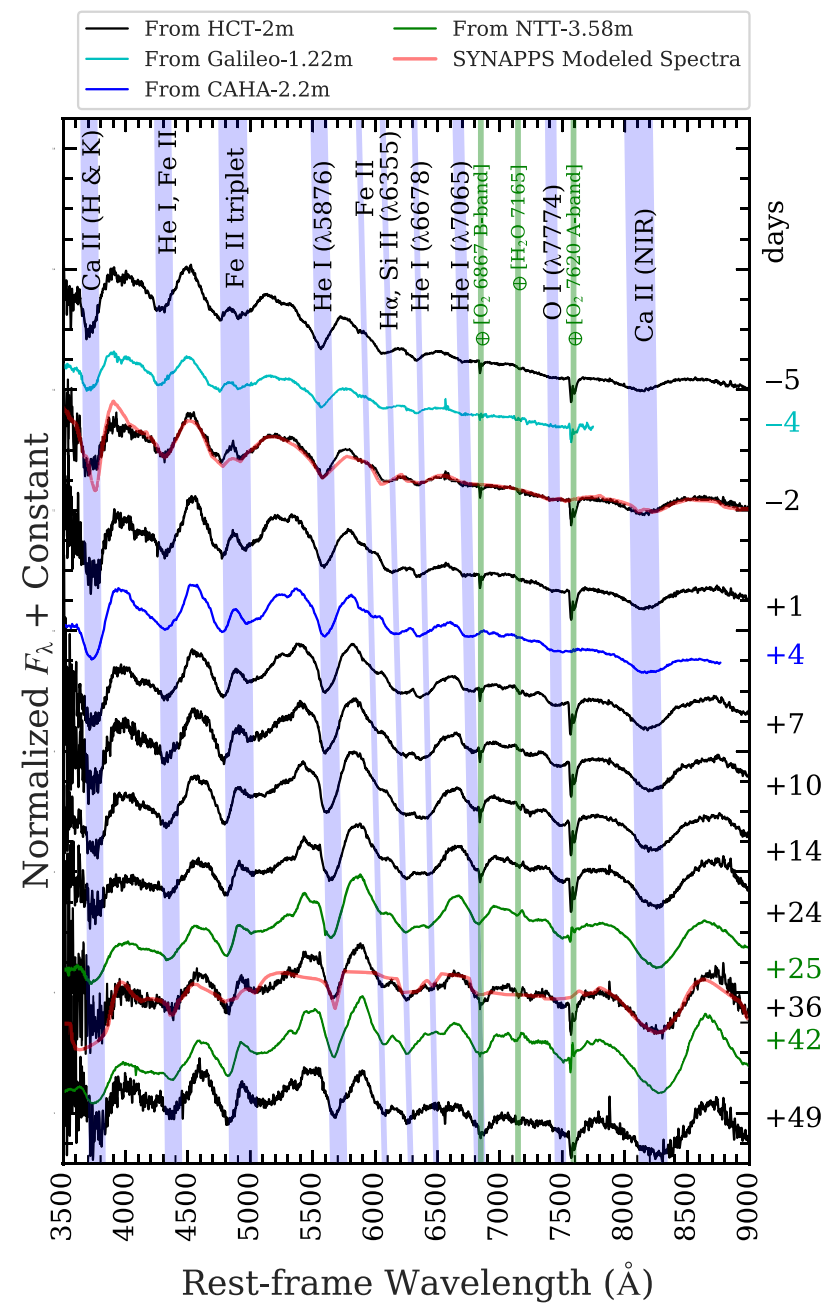

Figure 9. Early photospheric phase spectra of SN 2012au, from $\sim-5$ to +49 d. The grey bands trace the evolution of absorption troughs of various elements, and indicate decreasing velocity with time. The elements and their rest-frame wavelengths are written at the top; telluric features are also shown, as bands with green colour.

on NTT-3.58m. Spectroscopic data reduction was performed in a standard manner, as described in Kumar et al. $(2020,2021)$. The spectra of SN 2012au are subdivided into three different phases: early photospheric phase (from $\sim-5$ to $+50 \mathrm{~d}$; 14 spectra), late photospheric phase (from $\sim+60$ to $+108 \mathrm{~d} ; 7$ spectra), and nebular phase (at $+391 \mathrm{~d} ; 1$ spectrum). The early photospheric phase spectra, along with two modelled spectra (in red), are plotted in Fig. 9. The modelled spectra (at -2 and $+36 \mathrm{~d}$; in red) are reproduced using SYNAPPS (an automated version of the SYN++ code: Thomas et al. 2011) and are presented with individual ion contributions in Fig. 10. The late photospheric phase spectra and line velocity evolution are shown in Figs 11 and 12, respectively. The nebular phase spectrum at $+391 \mathrm{~d}$ is shown along with two published nebular spectra (at +275 and +323 d; Milisavljevic et al. 2013) in Fig. 13. The remaining unpublished NIR spectra of SN 2012au (at -5 and $+21.8 \mathrm{~d}$ ), along with three published ones $($ at $+32,+82$, and +319 d) taken from Milisavljevic et al. (2013), are also presented. All spectra were corrected for total extinction (Galactic + host) of $E(B-V)=0.063$ mag and also shifted to rest-frame wavelengths. 


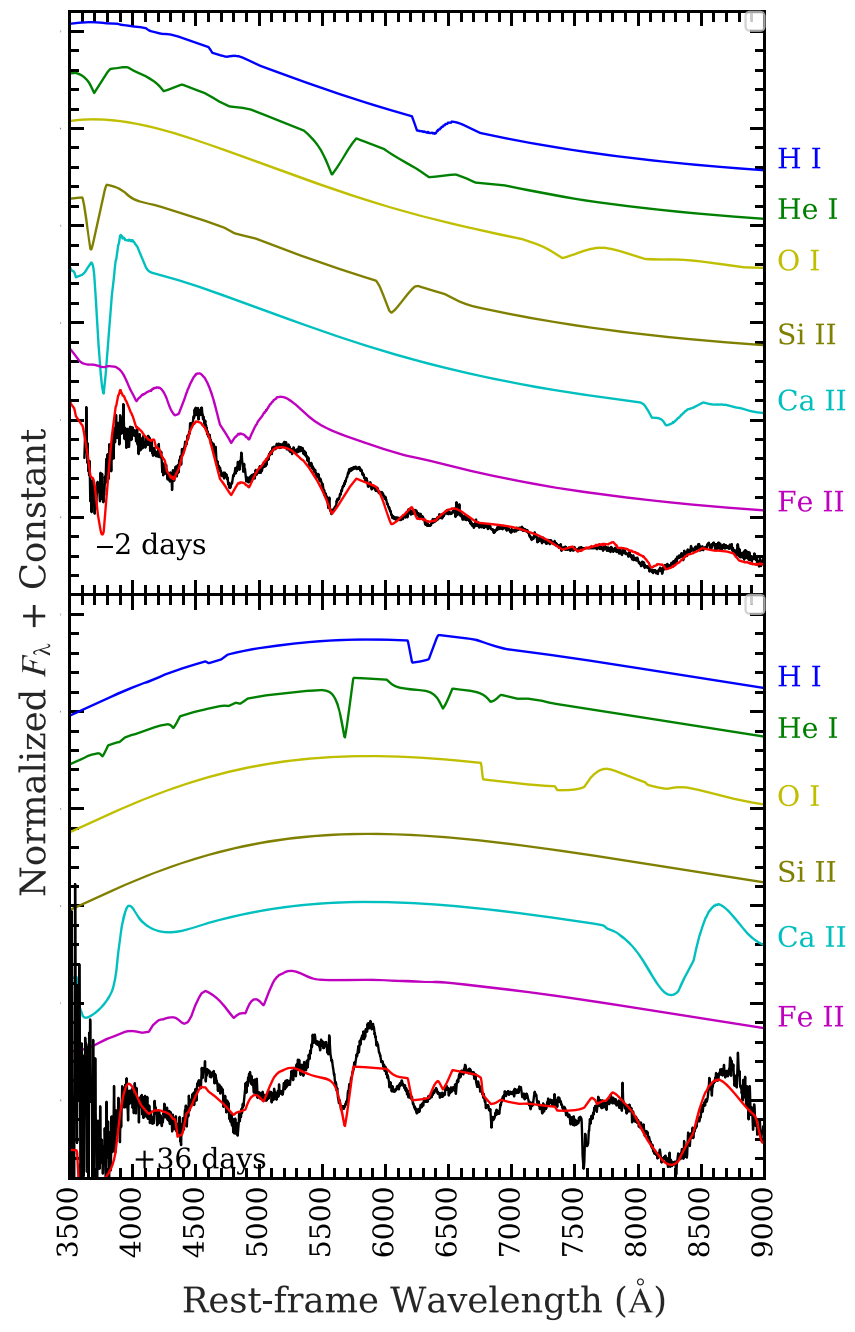

Figure 10. Observed and synthetic spectra of SN 2012au at -2 and $+36 \mathrm{~d}$ are shown. The contribution of individual ions to generate the best-matchimg synthetic spectrum using the SYNAPPS (Thomas et al. 2011) code is plotted and accounts for $\mathrm{H} \alpha$, He I, O I, Si II, Ca II, and Fe II elements.

\subsection{Optical spectroscopic evolution at photospheric phase}

Early and late photospheric phase spectra of SN 2012au are plotted in Figs 9 and 11, respectively. The first spectrum of SN 2012au was observed at $-5 \mathrm{~d}$, showing a blue continuum with a temperature of $\approx 9000 \mathrm{~K}$. The lines are identified following Milisavljevic et al. (2013), Takaki et al. (2013), and SYNAPPS spectral matching (Thomas et al. 2011). To construct the basic chemical composition, we performed SYNAPPS spectral matching for the photospheric phase spectra at -2 and $+36 \mathrm{~d}$ (in red). Thereafter, $\mathrm{SYN}++$ has been used to get the contribution of individual ions from SYNAPPS bestmatching synthetic spectra (see Fig. 10). To generate the bestmatched synthetic spectrum at $-2 \mathrm{~d}$, we used a $v_{\mathrm{ph}}$ of $\approx 14100 \mathrm{~km} \mathrm{~s}^{-1}$ and photospheric temperature of $\approx 7000 \mathrm{~K}$. At this epoch, He I, Ca II, and $\mathrm{Fe}$ II are found to be the most prominent features, with minor contributions from $\mathrm{H} \alpha$, O I, and $\mathrm{Si}$ II; see the top panel of Fig. 10. The conspicuous absorption near $6200 \AA$ in the early photospheric phase spectra of SN 2012au is possibly $\mathrm{H} \alpha$, as observed in a sample of SNe Ib by Elmhamdi et al. (2006). This favours considerable mixing of $\mathrm{H}$ in the He envelope (Maurer et al. 2010). The absorption minima of $\mathrm{H} \alpha, \mathrm{He}$ I, and Si II are usually fitted with larger velocities than other

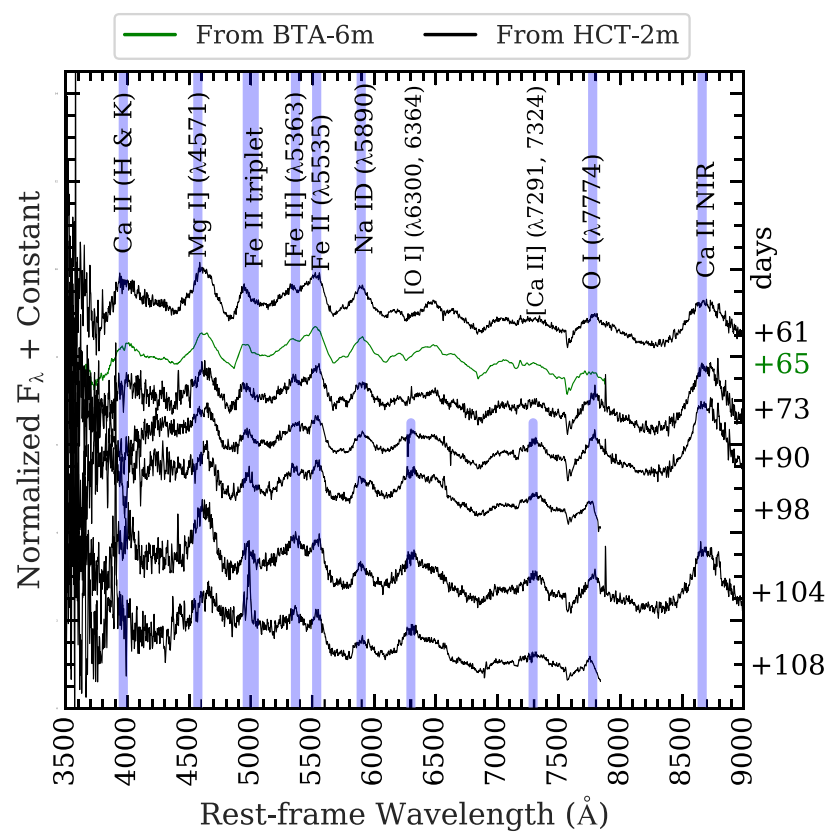

Figure 11. The late photospheric phase spectra of SN $2012 \mathrm{au}$ from $\sim+60$ to $+108 \mathrm{~d}$ are plotted. The grey colour bands represent the rest-frame wavelengths (written on the top) of various elements. The [OI] doublet and [Ca II] start a little later in comparison with other emission features.

features. To generate the spectrum at $+36 \mathrm{~d}$ using SYNAPPS, similar ions to those used for the spectrum at $-2 \mathrm{~d}$ were used (see lower panel of Fig. 10). We obtained $v_{\text {ph }}$ of $\approx 8000 \mathrm{~km} \mathrm{~s}^{-1}$ and a photospheric temperature of $\approx 4750 \mathrm{~K}$ to regenerate the spectrum at $+36 \mathrm{~d}$.

Early photospheric spectra of SN 2012au have absorption features of He I $(\lambda \lambda 5876,6678$, and $\lambda 7065)$, O I $(\lambda 7774)$, Si II $(\lambda \lambda 3838,6355)$, Ca II (H\&K and NIR), and Fe II ( $\lambda \lambda \lambda 4549,4925,5018$, and $\lambda 5169)$, however He I is the most prominent one; see Figs 9 and 10. Spectra up to $\sim+50 \mathrm{~d}$ exhibit a clear absorption feature of O I $\lambda 7774$ but its profile is contaminated by the telluric $\oplus\left[\mathrm{O}_{2} \lambda 7620\right]$ band. The feature at around $\sim 3700 \AA$ is attributed as a blend of Ca II $\mathrm{H} \& \mathrm{~K}$ and Si II $\lambda 3838$. The Ca II NIR triplet is possibly blended with O I $\lambda 8446$ and appears to evolve, becoming more prominent with time (see Fig. 10). Throughout the early photospheric phase, the $\sim 4000-5500 \AA$ window is primarily dominated by Fe II ions. The Fe II $(\lambda \lambda 4925,5018$, and $\lambda 5169)$ triplet is prominent, while the absorption feature at $\sim 4300 \AA$ seems to be a blend between Fe II $\lambda 4549$ and $\mathrm{He} \mathrm{I} \lambda 4471$. The blue wing of the $\mathrm{H} \alpha$ line is contaminated by a weak feature that could be Si II $\lambda 6355$, observed at around 6050 $\AA$ (Fig. 10). In the spectrum at $\sim-4 \mathrm{~d}$, absorption troughs of all elements are blueshifted and show $v_{\text {ph }}$ of nearly $\sim 15000 \mathrm{~km} \mathrm{~s}^{-1}$. However, with time the absorption features move towards their restframe wavelengths (indicating decreasing velocity), as shown by the grey coloured inclined bands.

As spectra evolve from early to late photospheric phase, the absorption features start to disappear and emission-line components emerge (see Fig. 11). The emission lines are indicated with vertical grey bands. O I $\lambda 7774, \mathrm{Na}$ ID, Mg I], Ca II H\&K and NIR, and the Fe II triplet appear earlier than the [O I] $\lambda \lambda 6300,6363$ doublet and [Ca II] $\lambda \lambda 7291,7324$ (hereafter [Ca II] $\lambda 7300$ ). The emission feature at $\sim 5500 \AA$ is possibly a blend of [O I] $\lambda 5577$ and [Fe II] $\lambda 5363$. Initially, the [O I] doublet appears to be flat-topped, whereas in later epochs it becomes asymmetric, with a suppressed redder component. 


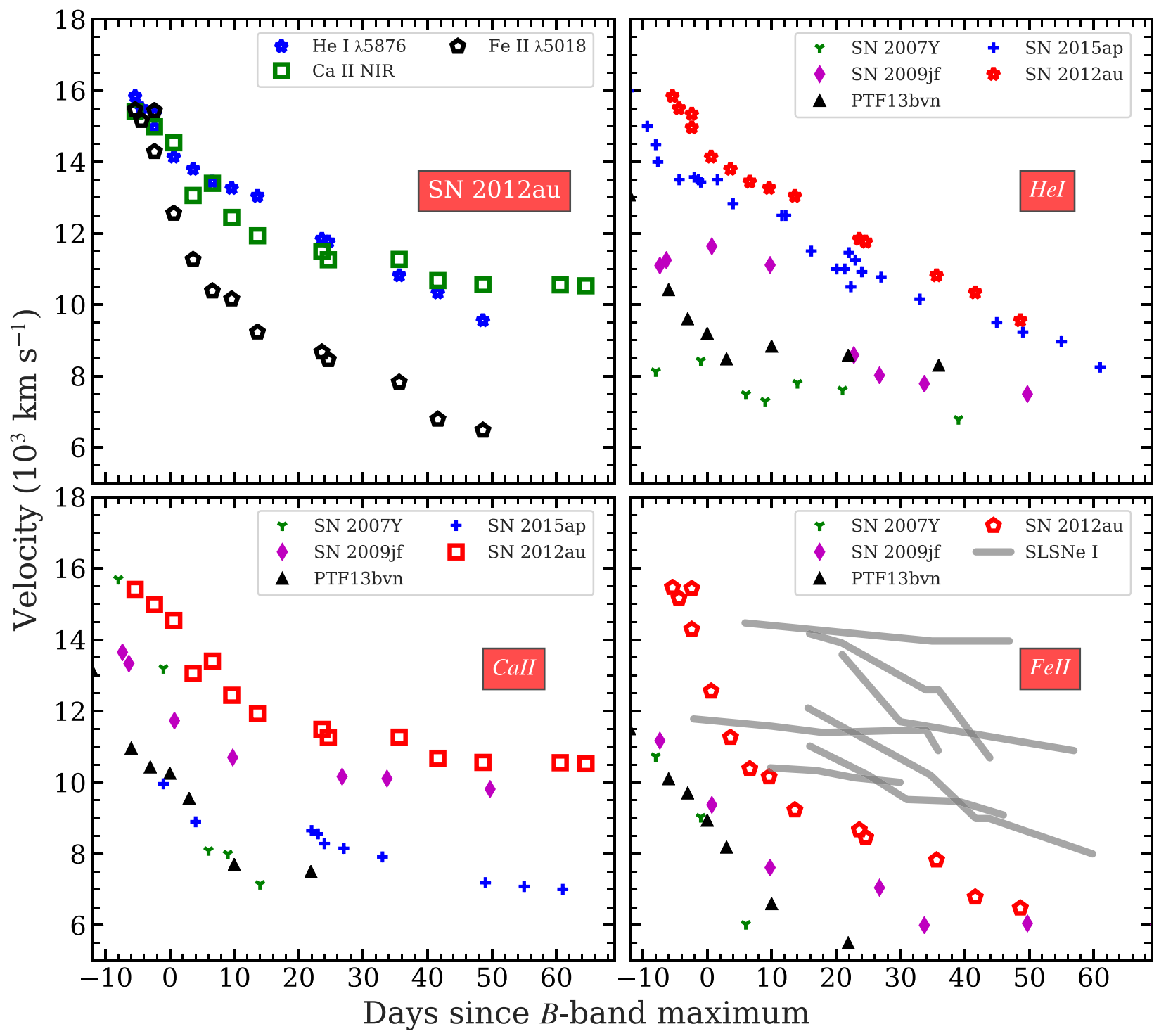

Figure 12. Upper left: He I, Ca II NIR, and the Fe II line velocities of SN 2012au. The line velocities of SN 2012au are also compared with those of SN 2007Y (Stritzinger et al. 2009), SN 2009jf (Sahu et al. 2011), iPTF13bvn (Srivastav, Anupama \& Sahu 2014), and SN 2015ap (Prentice et al. 2019; Aryan et al. 2021) in the other three panels.

\subsubsection{Ejecta mass, photospheric radius, and optical depth}

The photospheric radius around the peak $\left(r_{\mathrm{ph}}\right)$, total optical depth below the photosphere around maximum $\left(\tau_{\text {total }}\right)$, and $M_{\mathrm{ej}}$ of the $\mathrm{SN}$ can be constrained using $v_{\mathrm{ph}}$ and the time since the explosion. Assuming homogeneous expansion, $r_{\mathrm{ph}}$ can be estimated using $v_{\mathrm{ph}}$ and the time of explosion $\left(t_{\exp }\right)$ as

$r_{\mathrm{ph}}=v_{\mathrm{ph}} \times \frac{\left(t-t_{\mathrm{exp}}\right)}{(1+z)}$,

where $t$ is the time of the observation and $t_{\exp }$ is the time of explosion. We also adopt

$\tau_{\text {total }} \approx 3 c / v_{\mathrm{ph}}$,

where $c$ is the speed of light; the details are discussed in KonyvesToth et al. (2020), see also Arnett (1996) and Branch \& Wheeler (2017). Finally, to estimate $M_{\mathrm{ej}}$ using $v_{\mathrm{ph}}$ and $\tau_{\text {total }}$, equation (8) of
Konyves-Toth et al. (2020) has been used:

$M_{\mathrm{ej}}=\frac{4 \pi}{3} \frac{v_{\mathrm{ph}}^{2} \times\left(t-t_{\mathrm{exp}}\right)^{2}}{(1+z)^{2}} \frac{\tau_{\mathrm{total}}}{\kappa}$.

To calculate $M_{\mathrm{ej}}$ using the above equation, a value of $\kappa=0.05$ $\mathrm{cm}^{2} \mathrm{~g}^{-1}$ was chosen. For SN 2012au, the near-peak spectrum at -2 $\mathrm{d}$ (14.5 d since explosion) was modelled with a $v_{\mathrm{ph}}$ of $\sim 14100 \mathrm{~km}$ $\mathrm{s}^{-1}$. Using these equations, the values of $r_{\text {phot }}, \tau_{\text {total }}$, and $M_{\mathrm{ej}}$ are $\sim 1.8$ $\times 10^{15} \mathrm{~cm}, \sim 63.8$, and $\sim 8.3 \mathrm{M}_{\odot}$. Values of $v_{\mathrm{ph}}$ and the time since explosion discussed here correspond to $E_{k} \sim 5.4 \times 10^{51} \mathrm{erg}$. For SN 2012au, the values of $r_{\mathrm{ph}}$ and $M_{\mathrm{ej}}$ are lower and $\tau_{\text {total }}$ is higher than for SLSNe I: SN 2010kd (Kumar et al. 2020) and SN 2019neq (Konyves-Toth et al. 2020). 


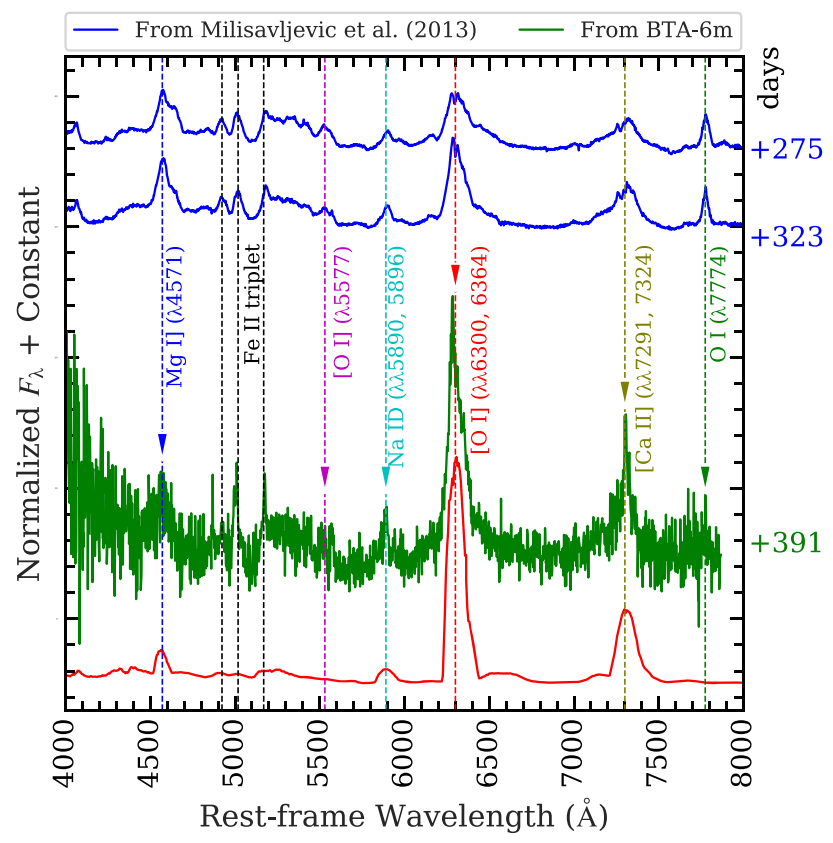

Figure 13. The nebular phase spectrum of SN 2012au at $+391 \mathrm{~d}$ is plotted along with two published spectra at +275 and $+323 \mathrm{~d}$ (taken from Milisavljevic et al. 2013). The dotted vertical lines represent the rest-frame wavelengths of various elements. The modelled spectrum generated using $M_{\text {ZAMS }}=17 \mathrm{M}_{\odot}$ is also shown in red for comparison (Jerkstrand et al. 2015).

\subsubsection{Line velocities}

The evolution of line velocities in the rest-frame spectra of SN 2012au is estimated by fitting a Gaussian profile to the absorption components. The evolution of the velocity for He I $\lambda 5876$, the Ca II NIR triplet (rest-frame wavelength is taken at $\lambda 8571$ ), and Fe II $\lambda 5169$ lines is shown in Fig. 12 (upper left panel). The velocities of the $\mathrm{He}$ I and Fe II lines are measured only until $\sim+50 \mathrm{~d}$, because at later phases their profiles have blended with other lines. Before the $B$ band maximum (at $-4 \mathrm{~d})$, the three features have nearly the same velocity $\left(\sim 15000 \mathrm{~km} \mathrm{~s}^{-1}\right)$. The $v_{\mathrm{ph}}$ estimated using SYNAPPS in the $-2 \mathrm{~d}$ spectrum $\left(\sim 14100 \mathrm{~km} \mathrm{~s}^{-1}\right)$ is in good agreement with the value obtained from measuring the position of the Fe II absorption trough. For this reason, we consider the Fe II velocities as representative for the evolution of $v_{\mathrm{ph}}$. The Fe II line velocity declines to $\sim 12500 \mathrm{~km}$ $\mathrm{s}^{-1}$ at maximum, and exhibits lower velocities than $\mathrm{He}$ I and $\mathrm{Ca}$ II at later phases. We note that the velocities estimated in this study for the different ions in the spectra of SN 2012au are closer to those reported by Takaki et al. (2013).

From peak to $\sim+60 \mathrm{~d}$, the velocity of He I decreases from $\sim 15000$ to $8000 \mathrm{~km} \mathrm{~s}^{-1}$. In the case of SN 2012au, it remains higher than the average He I velocities estimated for a sample of SNe Ib by Fremling, Sollerman \& Kasliwal (2018). Until $\sim+45$ d, the Ca II NIR triplet seems to have a similar velocity to the He I line, but it decays more slowly at later phases, at a nearly constant velocity of $\sim 11000 \mathrm{~km}$ $\mathrm{s}^{-1}$. He I and the Ca II NIR triplet show higher velocities than Fe II, suggesting that the former lines are generated in the outer parts of the ejecta, while Fe II lines form in the inner layers. We also note that, up to $\sim+25 \mathrm{~d}$, the $v_{\text {ph }}$ (Fe II line velocity) of SN 2012au is found to be higher than the average $v_{\mathrm{ph}}\left(8000 \pm 2000 \mathrm{~km} \mathrm{~s}^{-1}\right)$ estimated at maximum light for a sample of $\mathrm{SNe} \mathrm{Ib} / \mathrm{c}$ (Cano 2013).

In the other three panels of Fig. 12, we compare the He I, Ca II NIR, and Fe II line velocities of SN 2012au (red line) with those of
SN 2007Y (green: Stritzinger et al. 2009), SN 2009jf (magenta: Sahu et al. 2011), iPTF13bvn (black: Srivastav et al. 2014), and SN 2015ap (blue: Prentice et al. 2019; Aryan et al. 2021). The Fe II line velocity for SN 2015ap is not available because of contamination by host galaxy lines (Prentice et al. 2019). Across the entire evolution, the $\mathrm{He}$ I and Ca II line velocities of SN 2012au remain higher than those of the other SNe Ib discussed, whereas the Fe II line velocity of SN 2012au is higher only until $\sim+30 \mathrm{~d}$, and thereafter it is nearly equal to that of SN 2009jf. In the lower right panel of Fig. 12, the Fe II ion velocities of SNe Ib along with SN 2012au have been compared with those estimated for SLSNe I (in grey) by Nicholl et al. (2015). Similarly to Type Ic in Nicholl et al. (2015), SN 2012au and other plotted SNe Ib exhibit faster decaying Fe II velocities in comparison with SLSNe I.

\subsection{Optical spectroscopic evolution in the nebular phase}

In the nebular phase, ejecta become optically thin and the deeper layers are probed, and the available spectral features are used to investigate the geometry of the ejecta and other physical parameters (Taubenberger et al. 2009). Therefore, to trace the evolution of emission lines at late phases, the spectrum of SN 2012au at $+391 \mathrm{~d}$ (in green colour) along with two publicly available spectra at +275 and +323 d (in blue colour; taken from Milisavljevic et al. 2013) is investigated and plotted in Fig. 13. The nebular spectra of SN 2012au are dominated by the semi-forbidden $\mathrm{Mg} \mathrm{I}] \lambda 4571$, forbidden [O I] $\lambda \lambda 6300,6364$ doublet, and [Ca II] $\lambda \lambda 7300$ features, along with weaker Fe II triplet, [O I] $\lambda 5577, \mathrm{Na}$ I D, and O I $\lambda 7774$ lines. All the features discussed above are marked with vertical dotted lines in Fig. 13. The $\mathrm{Mg}$ I], Na I D, and [Ca II] lines do not evolve significantly between +323 and $+391 \mathrm{~d}$, in contrast to the [O I] doublet, which shows major evolution. While at +275 and $+323 \mathrm{~d}$ the $[\mathrm{O} \mathrm{I}] \lambda 5577$ and $\mathrm{O} \mathrm{I}$ $\lambda 7774$ features are evident, those become weaker at $+391 \mathrm{~d}$.

The nebular phase spectrum of SN 2012au at $+391 \mathrm{~d}$ is also compared with the modelled nebular spectra published by Jerkstrand et al. (2015) for SNe IIb. After $\sim+150$ d, the spectra published by Jerkstrand et al. (2015) can also be compared with SNe Ib because at these late phases the influence of the $\mathrm{H}$ envelope is negligible. We tried to match the spectrum of SN 2012au at $+391 \mathrm{~d}$ with all modelled spectra published by Jerkstrand et al. (2015). However, we found that the modelled spectrum generated for $M_{\text {ZAMS }}$ of $17 \mathrm{M}_{\odot}$ considering strong mixing and dust (in red) matched well with the +391 d spectrum of SN 2012au (see Fig. 13). The prominent Mg I], [OI] doublet, and [CaII] emission lines in the synthetic spectrum matched the observations well.

\subsection{Emission-line study}

The geometry of the SN ejecta can be constrained from the emissionline profiles and observed fluxes in nebular phase spectra, mainly using the isolated [O I] doublet (Taubenberger et al. 2009; Fang et al. 2019). In Fig. 14, we present the evolution of $\mathrm{Mg} \mathrm{I}$ ], [O I] doublet, and [Ca II] lines in the velocity domain. The zero velocities for $\mathrm{Mg} \mathrm{I}]$, [O I] doublet, and [Ca II] lines are taken at $\lambda 4571, \lambda 6300$, and $\lambda 7291$, respectively. Up to $+108 \mathrm{~d}, \mathrm{Mg} \mathrm{I}]$ has a redshifted maximum, but from $\gtrsim+275 \mathrm{~d}$ it appears to be at zero velocity. The [Ca II] emission lines are redshifted throughout the nebular phase $\left(\sim 500-1200 \mathrm{~km} \mathrm{~s}^{-1}\right.$, see Fig. 14), though a possibility of [Ca II] $\lambda$ blending with [O II] $\lambda \lambda 7320$, 7331 also exists. As discussed earlier, the [O I] doublet profile is flattopped at around $\sim+90 \mathrm{~d}$, attributed to the blending of $\lambda 6300$ and $\lambda 6363$ features. However, at later phases (from $+275 \mathrm{~d}$ ), the core becomes narrower, superposed on a broader base. At $+275 \mathrm{~d}$, the 

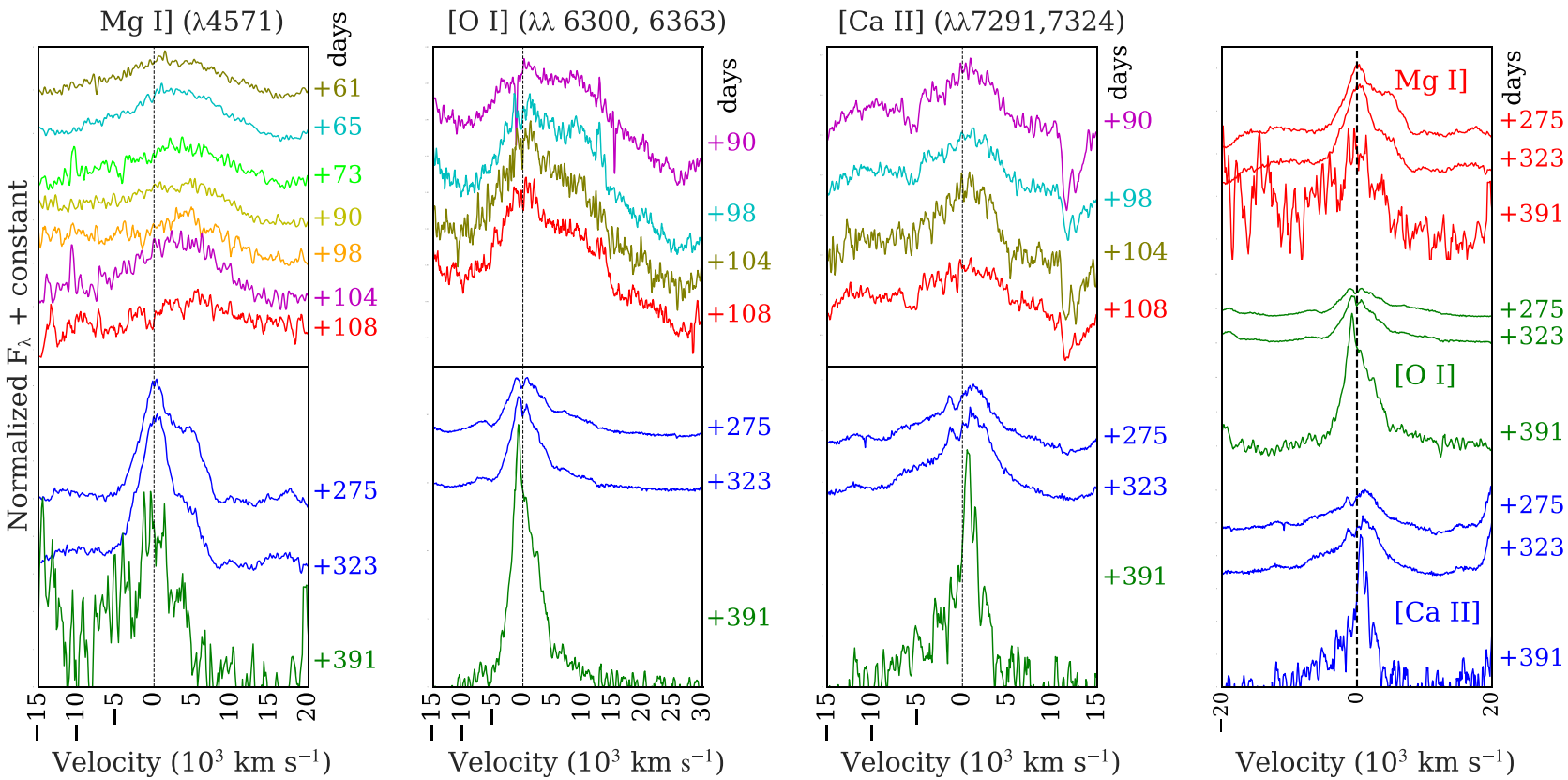

Figure 14. Evolution of $\mathrm{Mg}$ I], [O I] doublet, and [Ca II] lines plotted in the velocity domain, with their zero velocities being taken at $\lambda 4571, \lambda 6300$, and $\lambda 7291$, respectively.

[O I] doublet profile shows two peaks of nearly equal intensity with $\delta \lambda \sim 40 \AA$, although at $+323 \mathrm{~d}$ the redder component of the double peak appears suppressed and $\delta \lambda$ decreases to $\sim 30 \AA$. The suppression of the redder component increases with time, as can be determined from the spectra at +391 and $+2270 \mathrm{~d}$ (Milisavljevic et al. 2018). This suppression of the redder component may point to large-scale clumps in the oxygen ejecta at late phases. On the other hand, the blue wing of the [O I] doublet seems blueshifted by about $\sim 1100 \mathrm{~km}$ $\mathrm{s}^{-1}$ at $+275 \mathrm{~d}$, whereas with time it approaches zero velocity and presents blueshiftings of $\sim 750$ and $700 \mathrm{~km} \mathrm{~s}^{-1}$ in the spectra at +391 and $+2270 \mathrm{~d}$, respectively (Milisavljevic et al. 2018). The blueshifted [O I] doublet profile indicates that photons were emitted near the side of the ejecta (Maeda et al. 2008). Plausible reasons behind the blueshifted narrow peak of the [O I] doublet on a broader base are large-scale clumping, a unipolar jet, or a single massive blob moving towards the observer (Mazzali et al. 2001; Maeda et al. 2002, 2006; Taubenberger et al. 2009). The rightmost panel of Fig. 14 shows that, in the spectral profiles, $\mathrm{Mg}$ I], [O I] doublet, and [Ca II] features at $+275,+323$, and $+391 \mathrm{~d}$ are asymmetric around zero velocity, which indicates clearly that synthesized elements are being distributed asymmetrically or clumping occurs in the ejecta of SN 2012au (Taubenberger et al. 2009).

\subsection{1 $\left[\mathrm{O}_{\mathrm{I}}\right] \lambda 5577$ and $\left[\mathrm{O}_{\mathrm{I}}\right] \lambda \lambda 6300,6364$ doublet}

The [O I] emission lines in the nebular spectra are used to constrain $M_{\mathrm{O}}, M_{\mathrm{ZAMS}}$, and $M_{\mathrm{He}}$. We derived $M_{\mathrm{O}}$ in units of $\mathrm{M}_{\odot}$ using the following formula from Uomoto (1986):

$M_{\mathrm{O}}=10^{8} \times D^{2} \times F([\mathrm{O} \mathrm{I}]) \times \exp \left(2.28 / T_{4}\right)$.

Here, $D$ represents the host galaxy distance in Mpc (23.5 for NGC 4790; Milisavljevic et al. 2013), F([O I]) is the total flux of the [O I] doublet in units of erg s $\mathrm{s}^{-1} \mathrm{~cm}^{-2}$, and $T_{4}$ is the temperature of the oxygen-emitting region in $10^{4} \mathrm{~K}$. The formula discussed above is applicable in the high-density and low-temperature regime, and the ejecta of SNe Ib favour these conditions (Leibundgut et al. 1991; Elmhamdi et al. 2004). The flux ratio of [OI] $\lambda 5577$ and the [O I] doublet is dependent on the temperature and optical depth (Osterbrock 1989), hence the assumption of an optically thin regime is used to calculate the O I temperature. In the case of SN 2012au, [O I] $\lambda 5577$ is not detected clearly in the spectrum at $+391 \mathrm{~d}$; however, the spectrum at $+323 \mathrm{~d}$ (taken from Milisavljevic et al. 2013) exhibits significant [O I] $\lambda 5577$ emission and can be used to estimate the O I temperature. Before calculating the flux values, the spectrum has been scaled to the photometric flux. We infer $F([\mathrm{OI}] \lambda 5577)$ $\approx(1.18 \pm 0.56) \times 10^{-14}$ and $F([\mathrm{O}$ I $]$ doublet $) \approx(1.12 \pm 0.07) \times$ $10^{-13} \mathrm{erg} \mathrm{s}^{-1} \mathrm{~cm}^{-2}$. Using the flux values estimated above and equation (2) of Jerkstrand et al. (2014), we confer an O I temperature of $\approx 4098.39 \pm 309.15 \mathrm{~K}$, assuming $\beta_{\text {ratio }}=1.5$ (Jerkstrand et al. 2014). This corresponds to $M_{\mathrm{O}} \approx 1.62 \pm 0.15 \mathrm{M}_{\odot}$, which is higher than the values for Type Ib SNe tabulated in Table 1, and also higher than the $M_{\mathrm{O}}$ range $\left(0.1\right.$ to $\left.1.4 \mathrm{M}_{\odot}\right)$ estimated by Elmhamdi et al. (2004) for a sample of SESNe.

From $M_{\mathrm{O}}$, we can also derive $M_{\mathrm{ZAMS}}$ and $M_{\mathrm{He}}$. As proposed by Nomoto et al. (2006), for $M_{\mathrm{O}} \approx 0.16,0.77,1.05,2.35,3.22$, and $7.33 \mathrm{M}_{\odot}, M_{\text {ZAMS }}$ will be $15,18,20,25,30$, and $40 \mathrm{M}_{\odot}$, respectively, whereas $M_{\text {ZAMS }}$ of 13,15 , and $25 \mathrm{M}_{\odot}$ produce $M_{\mathrm{He}}$ of 3.3 , 4, and $8 \mathrm{M}_{\odot}$, respectively (Thielemann et al. 1996). As a consequence, a $M_{\text {ZAMS }}$ of $\sim 20-25 \mathrm{M}_{\odot}$ and $M_{\mathrm{He}}$ of $\sim 4-8 \mathrm{M}_{\odot}$ are inferred for SN 2012au.

\subsection{2 [Ca II] $\lambda 7300$ and $\left[\mathrm{O}_{I}\right] \lambda \lambda 6300,6364$ doublet}

$M_{\text {ZAMS }}$ can also be constrained using the flux ratio between [Ca II] $\lambda 7300$ and the $[\mathrm{O}$ I] doublet. The [Ca II]/[O I] flux ratio is weakly dependent on density and temperature, whereas its lower value indicates a higher core mass and hence a higher $M_{\text {ZAMS }}$ (Fransson \& Chevalier 1989; Elmhamdi et al. 2004; Fang \& Maeda 2019; Fang et al. 2019). In the case of SN 2012au, [Ca II]/[O I] increases from $\approx 0.5$ to 0.7 spanning between +108 and $+323 \mathrm{~d}$, thereafter decreasing to $\sim 0.3$ at 
+391 d. These [Ca II]/[O I] values for SN 2012au are close to those estimated for SN 1998bw (Patat et al. 2001; Kuncarayakti et al. 2015), SN 2002ap (Modjaz et al. 2014; Kuncarayakti et al. 2015), and SN 2009jf (Sahu et al. 2011). In the case of SN 2012au, the $[\mathrm{Ca}$ II $] /[\mathrm{O} \mathrm{I}]$ ratio of $\sim 0.3$ at $+391 \mathrm{~d}$ indicates a relative abundance of $\mathrm{Ca}$ II/O I $\approx(0.3$ to 1.0$) \times 10^{-3}$, as suggested by Fransson \& Chevalier (1989). The theoretical values of [Ca II]/[O I] were estimated by Fransson \& Chevalier (1989) for $M_{\mathrm{He}}$ and $M_{\text {ZAMS }}$ values from 2.68 $5.83 \mathrm{M}_{\odot}$ and $15-25 \mathrm{M}_{\odot}$, respectively. For SN 2012au, the range of [Ca II]/[O I] supports $M_{\mathrm{He}} \approx 5.83 \mathrm{M}_{\odot}$ and $M_{\mathrm{ZAMS}} \approx 25 \mathrm{M}_{\odot}$ under Model 1b of Fransson \& Chevalier (1989). Throughout the evolution (from +108 to $+391 \mathrm{~d}$ ), the $[\mathrm{Ca}$ II]/[O I] ratio values for SN 2012au are lower in comparison with those estimated for most of the SLSNe I and also suggest a $M_{\mathrm{He}}$ of $\approx 5.9 \mathrm{M}_{\odot}$ via comparison with the ratio from the SESN models of Jerkstrand et al. (2015) (see fig. 20 of Nicholl et al. 2019). Based on the [Ca II]/[O I] values, Kuncarayakti et al. (2015) conjectured a demarcation of SNe progenitors as binary (if $[\mathrm{Ca} \mathrm{II}] /[\mathrm{OI}]>0.7$ ) or a single WR star (if $[\mathrm{Ca} \mathrm{II}] /[\mathrm{O}$ I $]<0.7$ ). The range of $[\mathrm{Ca} \mathrm{II}] /[\mathrm{O} \mathrm{I}]$ flux ratio for SN 2012au is in agreement with the range of $[\mathrm{CaII}] /[\mathrm{OI}](\sim 0.3-0.7)$ for $\mathrm{SNe} \mathrm{Ib} / \mathrm{c}$ that are possibly produced by the explosion of a single massive WR star (Kuncarayakti et al. 2015).

\subsection{3 $\mathrm{Mg} I] \lambda 4571$ and $\left[\mathrm{O}_{I}\right] \lambda \lambda 6300,6364$ doublet}

Fig. 15 shows the evolution of the $\mathrm{Mg}$ I] and [O I] doublet flux ratios estimated using the nebular spectra of SN 2012au from +100 to +390 d. The Mg I]/[O I] evolution of SN 2012au is also compared with that of Type Ib/c SNe (taken from Hunter et al. 2009 and references therein) and some of the well-studied SLSNe I (taken from Nicholl et al. 2019 and references therein); see Fig. 15. A higher $\mathrm{MgI}] /[\mathrm{OI}]$ ratio is indicative of a higher degree of outer envelope stripping, as more of the $\mathrm{O}-\mathrm{Ne}-\mathrm{Mg}$ layer is exposed (Foley et al. 2003). From $\sim+100$ to $+275 \mathrm{~d}$, the $\mathrm{Mg}$ I $] /[\mathrm{O} \mathrm{I}]$ ratio of $\mathrm{SN} 2012$ au increases from $\sim 0.52$ to 0.9 , which is high in comparison with all Type $\mathrm{Ib} / \mathrm{c} \mathrm{SNe}$ and comparable to the sample of SLSNe I presented. However, at $+323 \mathrm{~d}, \mathrm{SN} 2012$ au exhibits the highest value of $\mathrm{Mg} \mathrm{I}] /[\mathrm{O} \mathrm{I}]$ ratio $(\sim 0.93)$. The increasing trend of the $\mathrm{Mg} \mathrm{I}] /[\mathrm{OI}]$ ratio of SN 2012au up to $+323 \mathrm{~d}$ may be attributed to an $\mathrm{Mg}$ I/O I abundance effect, symmetric ejecta, or non-symmetric ejecta viewed equatorially (Foley et al. 2003). On the other hand, from +323 to $+391 \mathrm{~d}$, the $\mathrm{Mg} \mathrm{I}] /[\mathrm{O} \mathrm{I}]$ ratio decreases to $\sim 0.27$, which is comparable to that of the other SESNe presented (see Fig. 15). The plausible reasons behind the decreasing $\mathrm{MgI}] /[\mathrm{OI}]$ trend observed at later epochs could be attributed to lack of high-density enhancements due to clumping, mixing, or asymmetry in the ejecta (Jerkstrand et al. 2015). However, dust formation and blending of $\mathrm{Mg} \mathrm{I}]$ with Fe II ions may also be other possible reasons.

\subsection{NIR spectroscopic evolution}

NIR spectroscopy and related studies have been performed for a good number of Type II SNe (e.g. SN 1998dl and SN 1999em: Spyromilio, Leibundgut \& Gilmozzi 2001), but only for a handful of SESNe so far (SN 2000ew (Ic): Gerardy et al. 2002; SN 2007gr (Ic): Hunter et al. 2009; LSQ13abf (Ib): Stritzinger et al. 2020; iPTF13bvn (Ib): Fremling et al. 2016; SN 2013ge (Ib/c): Drout et al. 2016; SN 2016adj (IIb/Ib): Banerjee et al. 2018; SN 2020oi (Ic) and SN 2020bvc (Ic): Rho et al. 2021). In this section, we present NIR spectroscopy of SN 2012au based on spectra at two epochs (at -5 and $+21.8 \mathrm{~d}$ since maximum) observed using SOFI on NTT-3.58m (see Table S5 in the supplementary material for the spectroscopic observations $\log$ ) along with three published NIR spectra (at +32 , $+82,+319$ d) taken from Milisavljevic et al. (2013); see Fig. 16. The spectra presented are in rest-frame wavelengths and also corrected for extinction using $E(B-V)=0.063 \mathrm{mag}$ (Milisavljevic et al. 2013), exhibiting a decrease in flux with wavelength, typically observed in SESNe. Line identifications of NIR spectra were done following Gerardy et al. (2002), Hunter et al. (2009), Banerjee et al. (2018), and Rho et al. (2021). The vertical red bands denote regions of poor atmospheric transparency. The first spectrum (at $-5 \mathrm{~d}$ ) appears featureless, with few lines due to intermediate-mass elements such as $\mathrm{He}$ I, C I, Mg I, O I, Na I, whereas, in the post-peak spectra, lines due to iron-group elements begin to appear. As the continuum flux decreases in late-time spectra, prominent features of the hydrogen Paschen series, Hi, He I, Na I, O I, MgI, Mg II, Si I, S I, and Ca I are traced, highlighted with vertical dotted lines in Fig. 16. Early spectra (up to +32 d) are mainly dominated by P Cygni profiles superimposed on a blue continuum, whereas in late-time spectra broad absorption features start appearing as the continuum fades; however, the spectrum at $+319 \mathrm{~d}$ is mainly dominated by emission features.

In the $J$ band $(\sim 10000-14000 \AA)$ region of all the spectra presented, the most prominent absorption feature near $\sim 10100 \AA$ is identified as very high-velocity He I $\lambda 10830$, see Fig. 16 . From -5 to $+82 \mathrm{~d}$, He I $\lambda 10830$ absorption features correspond to $v_{\mathrm{ph}}$ values from $\sim 20000-17000 \mathrm{~km} \mathrm{~s}^{-1}$, respectively. The He I $\lambda 10830$ velocity is higher for SN 2012au in comparison with that observed for SN 2016adj ( 14000 $\mathrm{km} \mathrm{s}^{-1}$ ) by Banerjee et al. (2018). However, we caution here that a small contribution from $\mathrm{P} \gamma$, C I $\lambda 10686$, Mg II $\lambda 10926$, and SiI $\lambda 10991$ to the He I $\lambda 10830$ feature could also be present, as also discussed by Milisavljevic et al. (2013); see also Medhi et al. (1996) and Wheeler et al. (1998). In the $J$ band region, all the spectra presented share nearly similar features except O I $\lambda 11291$. O I $\lambda 11291$ is clearly observed in the late-time spectra at +82 and +319 d, whereas it is absent in the spectra up to $+32 \mathrm{~d}$. In the $H$-band $(\sim 15000-18000 \AA)$ region, the premaximum spectrum of SN 2012au is nearly featureless, whereas prominent features of $\mathrm{Mg}$ I $\lambda \lambda \lambda 15025,15040,15048$, and $\lambda 15750$, Fe II $\lambda \lambda 16000$ and 16440, a blended feature of CI $\lambda 16895$ and $\operatorname{Mg}$ I $\lambda 17110$, and HI $\lambda 17370$ are present in the post-maximum spectra. In the $K$-band $(\sim 19000-24000 \AA)$ region also, the premaximum spectrum is almost featureless except for the prominent CaI $\lambda 19850$ line; on the other hand, the post-maximum spectra are dominated by $\mathrm{He}$ I $\lambda 20581$, a blended feature of $\mathrm{B} \gamma$ and $\mathrm{Mg} \mathrm{I}$ $\lambda 21150, \mathrm{Na}$ I $\lambda 22070, \mathrm{~S}_{\mathrm{I}} \lambda 22570, \mathrm{Ca}$ I $\lambda 23572$, and $\mathrm{Mg}$ I $\lambda 24820$ elements.

\subsubsection{Absence of $\mathrm{CO}$ emission}

Among SESNe, the first overtone of CO between 22900 and $24000 \AA$ was first detected by Gerardy et al. (2002) in the case of SN 2000ew (Type Ic) and later on in many others, including SN 2016adj (a Type IIb/Ib SN), showing CO features as early as $\sim 58 \mathrm{~d}$ since discovery (Banerjee et al. 2018). In the NIR spectra of SN 2012au at +82 and +319 d, a significant rising of the continuum has not been observed in the $K$ band (see Fig. 16), which indicates an absence of $\mathrm{CO}$ emission. The time-scale of $\mathrm{CO}$ formation depends on the synthesized $\mathrm{C} / \mathrm{O}$ mass, metal depletion, and level of mixing in the ejecta (Cherchneff \& Lilly 2008). Moreover, based on previous studies and limited examples (SN 2000ew, SN 2007gr, SN 2016adj, and SN 2020oi), the time-scales of CO formation in the case of 


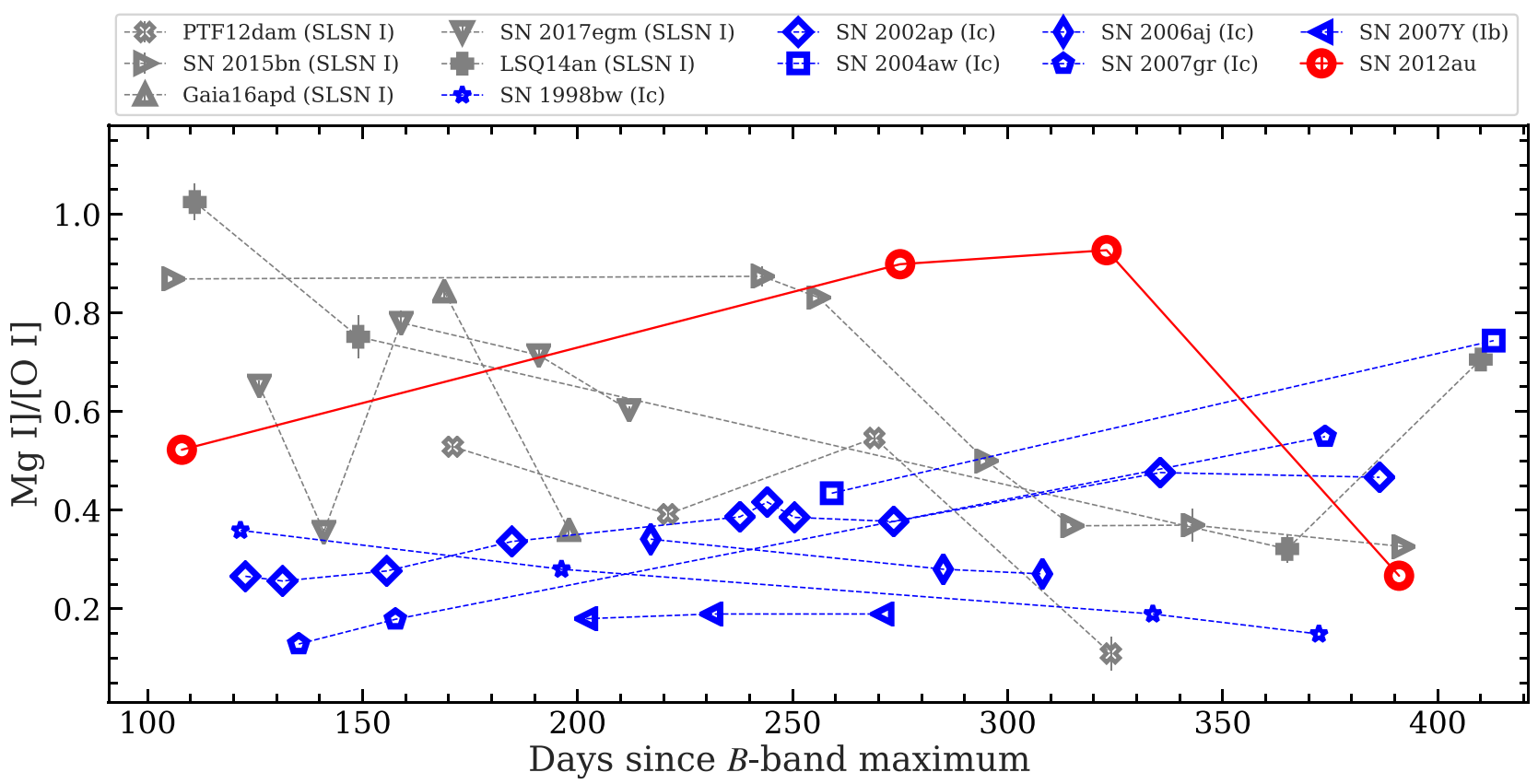

Figure 15. Evolution of the $\mathrm{Mg} \mathrm{I}] /[\mathrm{O} \mathrm{I}]$ ratio of SN 2012au compared with those found for SLSNe I (taken from Nicholl et al. 2019 and references therein) and other Type Ib/c SNe (taken from Hunter et al. 2009 and references therein).

SESNe appear to be shorter than those of Type IIP SNe (Banerjee et al. 2018; Sarangi, Dwek \& Arendt 2018).

The absence of CO molecule formation in the case of SN 2012au could be because of the high temperature of the ejecta (above molecule formation threshold) even at late phases. Another possible reason could be the higher mixing of ionized helium between ejecta layers, which can also hinder CO formation, as CO can be quickly destroyed by the presence of ionized helium (Gearhart et al. 1999; Gerardy et al. 2000; Cherchneff \& Dwek 2010). The presence of ionized helium in Type Ib SNe might therefore also have hampered $\mathrm{CO}$ formation, whereas this is not the case with Type Ic SNe (Sarangi \& Cherchneff 2013), as CO formation is a densitydependent process and a lower $\mathrm{C} / \mathrm{O}$ velocity is a requirement for $\mathrm{CO}$ formation. Hence it is possible that, due to a higher velocity, atmosphere density decreases, resulting in the prevention of $\mathrm{CO}$ formation (Gerardy et al. 2002). As higher $v_{\mathrm{ph}}$ is observed in SN2012au than in other presented SESNe (see Fig. 12), this may also be a possible reason behind the absence of $\mathrm{CO}$ emission in the NIR spectra of SN 2012au. CO emission also appears to be absent in the $+79 \mathrm{~d}$ spectrum of iPTF13bvn (Fremling et al. 2016).

\section{SPECTRAL COMPARISON OF SN 2012AU WITH OTHER SESNE}

Early and late photospheric spectra of SN 2012au (from +4 to +104 d, red colour) are compared with two well-studied SNe Ib: SN 2009jf (in green: Sahu et al. 2011; Valenti et al. 2011) and SN 2015ap (in blue: Aryan et al. 2021); see Fig. 17. The spectral evolution of the three $\mathrm{SNe} \mathrm{Ib}$ is similar, in particular the P Cygni profile and strength of He I at $\sim 5600 \AA$. Throughout the early photospheric phase, the absorption troughs of all elements in the SN 2012au spectra are highly blueshifted (higher velocities) compared with those of SN 2009jf and SN 2015ap. On the other hand, SN 2009jf seems to have narrower though faster-evolving features (Sahu et al. 2011). He I $\lambda \lambda 6678$ and 7065 features are present in the spectra of SN 2012au and SN 2015ap, whereas in the case of SN 2009jf He I $\lambda 6678$ is absent and He I $\lambda 7065$ is weaker. Overall, the early photospheric spectra of SN 2012au closely match those of SN 2015ap. The late photospheric spectra of the three $\mathrm{SNe}$ Ib exhibit the same forbidden lines; see the lower panel of Fig. 17. The main difference is in the evolution of [O I] $\lambda \lambda 6300$, 6363 and $[\mathrm{Ca} \mathrm{II}] \lambda \lambda 7291,7324$, which are weaker in SN 2012au. The [OI] doublet and the [Ca II] lines in the spectra of SN 2009jf and SN 2015ap at $\sim+80 \mathrm{~d}$ seem to be stronger in comparison with SN 2012au at $\sim+90 \mathrm{~d}$. This indicates that the photometrically slowevolving SN 2012au also evolves spectroscopically with longer timescales.

In Fig. 18, we compare the late nebular phase spectrum of SN 2012au (at +391 d) with some SNe Ib, Ic, and slow-decaying SLSNe I. Among SNe Ib, we chose SN 1996aq (Asiago archive), SN 2007Y (Stritzinger et al. 2009), and SN 2009jf (Sahu et al. 2011; Valenti et al. 2011); in SNe Ic, we chose SN 1998bw (IcBL + GRB: Patat et al. 2001), SN 2002ap (Ic-BL: Foley et al. 2003), and SN 2007gr (Ic: Shivvers et al. 2019), and from the slow-decaying SLSNe I, SN 2007bi (Gal-Yam et al. 2009) and SN 2015bn (Nicholl et al. 2016) have been chosen, based on the availability of very late nebular spectra.

In the upper panel of Fig. 18, the nebular spectrum of SN 2012au is compared with those of the three SNe Ib mentioned above. Notably, the [OI] and [Ca II] emission lines of SN 2012au match closely those of SN 2007Y. The spectra of SN 2012au and SN 2007Y show a single-peaked [OI], unlike SN 1996aq and SN 2009jf, the [OI] features of which have a double peak with different intensities. The [Ca II] emission peak of SN 2009jf appears to be blueshifted; on the other hand, SN 2012au presents a redshifted single emission peak. The [O I] $\lambda 7774$ feature is missing at these late phases in all $\mathrm{SNe} \mathrm{Ib}$ considered here. We also compare the nebular spectrum of SN 2012au with three SNe Ic, see the middle panel of Fig. 18. The three SNe Ic show similar lines to SN 2012au, although these lines are strongest in SN 2002ap (Ic-BL). SNe Ic exhibit weak emission lines of O I $\lambda 7774$, which is nearly absent in the case of SN 2012au. In the lower panel 


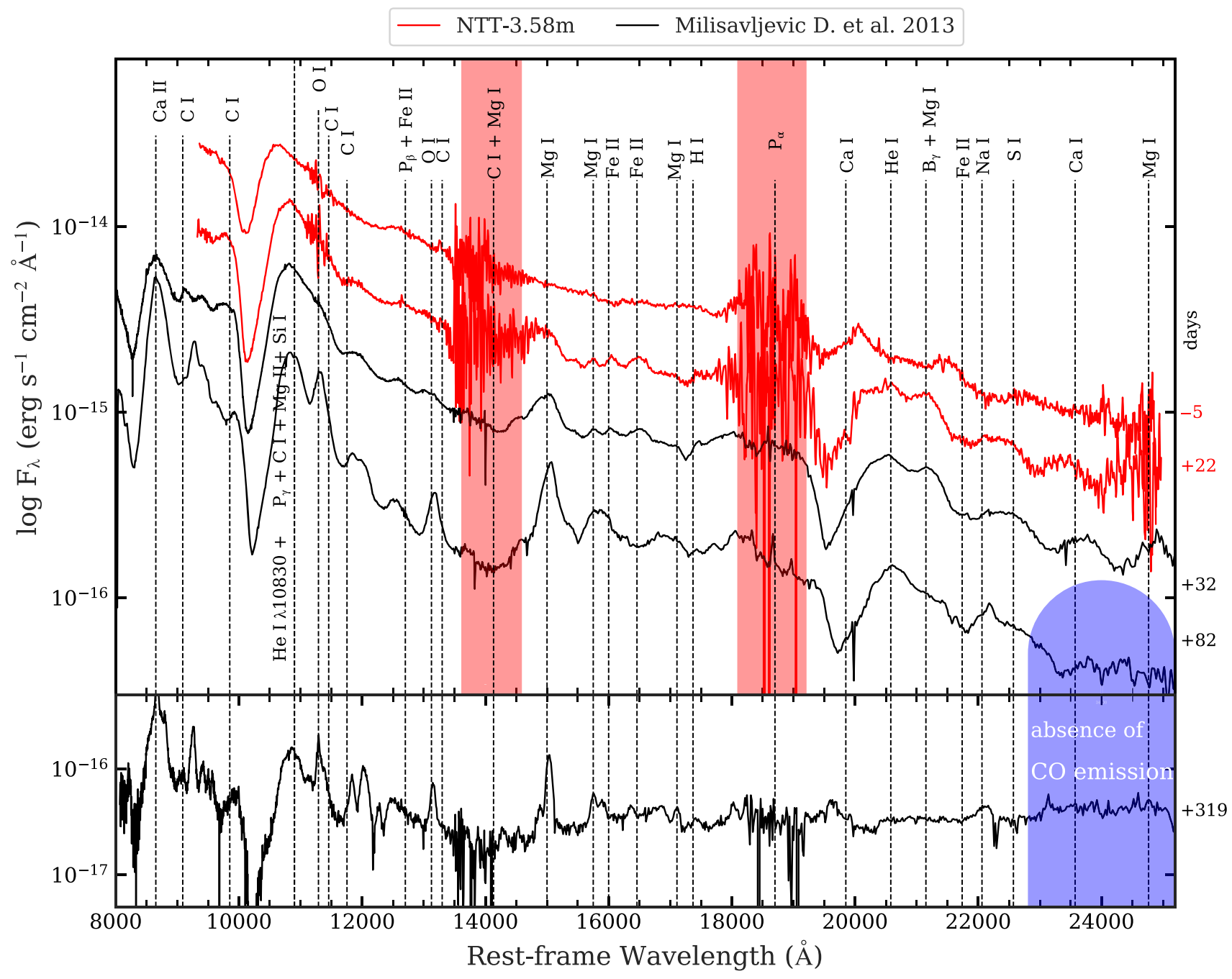

Figure 16. NIR spectra of SN 2012au obtained using SOFI on NTT-3.58m (at -5.1 and +21.8 d) and taken from Milisavljevic et al. 2013 (from +32 to +319 d) are presented. Prominent features are shown with vertical dotted lines, whereas vertical red bands denote regions of poor atmospheric transparency. Throughout the evolution, a prominent feature of He I $\lambda 10830$ and absence of the first overtone of $\mathrm{CO}$ can be seen.

of Fig. 18, we compare the nebular spectrum of SN 2012au with two slow-decaying SLSNe I. At such late phases, SLSNe I appear to share similar spectral features to $\mathrm{SNe} \mathrm{Ib} / \mathrm{c}$, as also suggested by Pastorello et al. (2010) and Quimby et al. (2018). SN 2012au and SLSNe I appear to have similar emission lines, although they are broader in SLSNe I. The emission lines evolve faster in the case of SN 2012au than in SN 2007bi and SN 2015bn, which have stronger OI $\lambda 7774$.

\section{PROGENITOR MODELLING USING MESA}

MESA is a hydrodynamical simulation code that evolves a certain ZAMS progenitor up to the stage where the core of the modelled progenitor is about to collapse. Further, the output of MESA is fed as input to STELLA and SNEC, which solve the radiative transfer equations and simulate the synthetic explosion. As a result of the synthetic explosion, supernova light curves and $v_{\mathrm{ph}}$ evolution, along with many other parameters, are generated. The light curves and $v_{\mathrm{ph}}$ evolution are then matched with the observed ones. Starting from the ZAMS, we are able to match the light curves and the photospheric velocities, so we have replicated the actual supernova explosion. Here, the explosions using STELLA and SNEC are complemented by the parameters obtained using MINIM. With a certain ZAMS mass progenitor, the explosion parameters in STELLA and SNEC are kept close to those obtained using MINIM. Thus, MESA is very useful to put important constraints on progenitor mass and other progenitor properties like metallicity, rotation, etc.

For SN 2012au, our analysis supports a progenitor mass of $\sim 20$ $25 \mathrm{M}_{\odot}$. Following Milisavljevic et al. (2013) and Kamble et al. (2013), where the metallicity $(Z)$ was determined using the methods of Sanders et al. (2012), and also using the N2 diagnostic of Pettini et al. (2004), we assume the metallicity at the site of SN 2012au to be around $1-2 \mathrm{Z}_{\odot}$. We modelled three non-rotating progenitor scenarios with $M_{\text {ZAMS }}$ of $20 \mathrm{M}_{\odot}, 22 \mathrm{M}_{\odot}$, and $25 \mathrm{M}_{\odot}$, each of them at three different $Z$ values of $0.02 \mathrm{Z}_{\odot}, 0.03 \mathrm{Z}_{\odot}$, and $0.04 \mathrm{Z}_{\odot}$. In all, we obtain a total of nine models. The model designation contains the mass and the $Z$ value. For instance, the M20_Z0.02 model represents a progenitor of $M_{\text {ZAMS }}=20 \mathrm{M}_{\odot}$ and with $Z=0.02 \mathrm{Z}_{\odot}$, while the M25_Z0.04 model indicates a progenitor of $M_{\text {ZAMS }} 25 \mathrm{M}_{\odot}$, with $Z=0.04 \mathrm{Z}_{\odot}$. One additional model, designated with the CSM mark, 


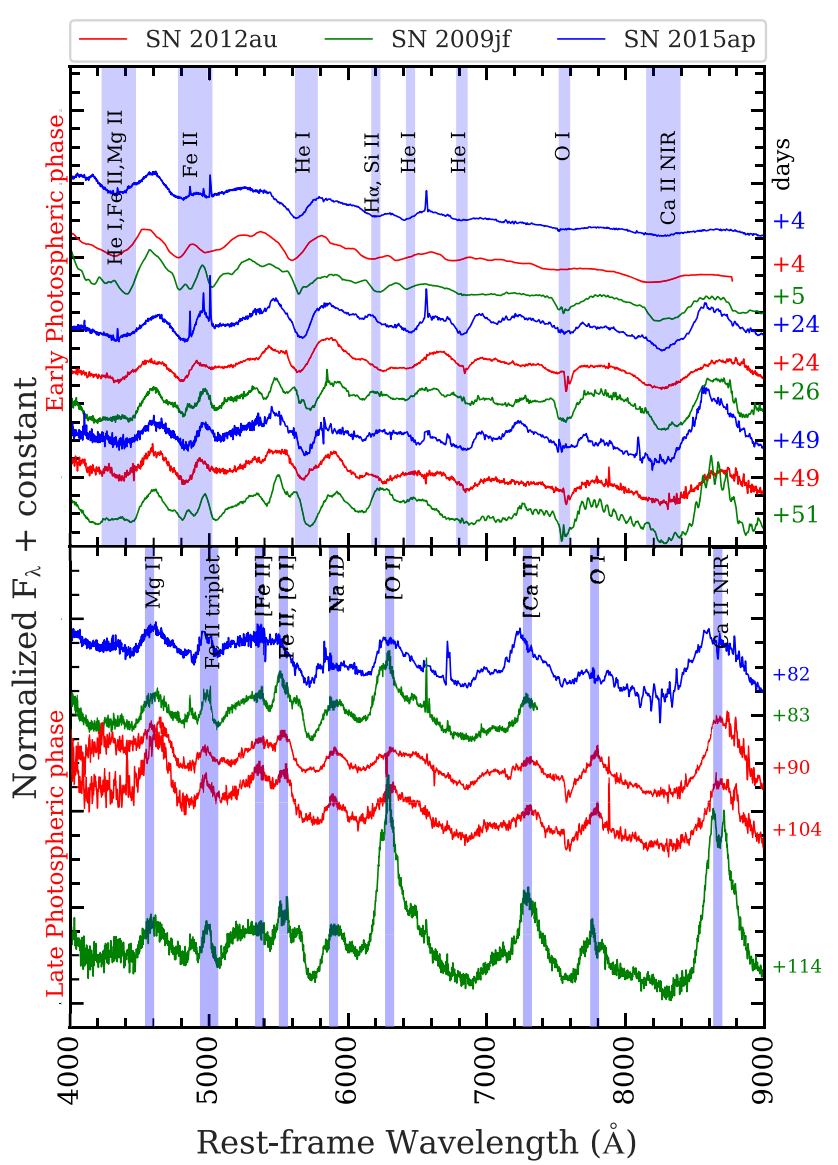

Figure 17. Early (from peak to $\sim+50 \mathrm{~d}$ ) and late (after $\sim+80 \mathrm{~d}$ ) photospheric spectra of SN 2012au compared with SN 2009jf and SN 2015ap at similar epochs in the upper and lower panels, respectively.

also includes the contribution of CSMI in the light curve. Using MESA (version 11701: Paxton et al. 2011, 2013, 2015, 2018), the models are evolved up to the stage of onset of rapid infall of the iron core. After excising the central core mass and when the shock is near breakout, we provide the corresponding output as input to the public version of STELLA (Blinnikov et al. 1998, 2000, 2006) included in MESA. STELLA evolves the model through shock breakout and beyond, generating the light curves and velocity evolution. Here, we briefly summarize the methods and assumptions for the different models.

Adopting the Ledoux criterion, we model the convection adopting the mixing theory of Henyey, Vardya \& Bodenheimer (1965). The mixing length parameter is set to be $\alpha=3.0$ in the region where the mass fraction of $\mathrm{H}$ is greater than 0.5 , and 1.5 in other regions. Following Langer, El Eid \& Fricke (1985), semi-convection is modelled with an efficiency parameter of $\alpha_{\mathrm{sc}}=0.01$. We follow Kippenhahn, Ruschenplatt \& Thomas (1980) for thermohaline mixing, with an efficiency parameter of $\alpha_{\text {th }}=2.0$. Convective overshooting is modelled with the diffusive approach of Herwig (2000), with $f=$ 0.01 and $f_{0}=0.004$ for both the convective core and shells. The DUTCH (Vink, de Koter \& Lamers 2001; Nugis \& Lamers 2000) scheme is used for the stellar wind, with a scaling factor of 1.0. $\mathrm{SNe} \mathrm{Ib}$ are assumed to originate from massive stripped-envelope stars, which have lost their outer $\mathrm{H}$ envelope via binary interactions (Yoon et al. 2010; Dessart et al. 2012; Eldridge \& Maund 2016; Ouchi \& Maeda 2017) or because of strong stellar winds (e.g. Gaskell et al. 1986; Eldridge, Langer \& Tout 2011; Groh et al. 2013). To strip

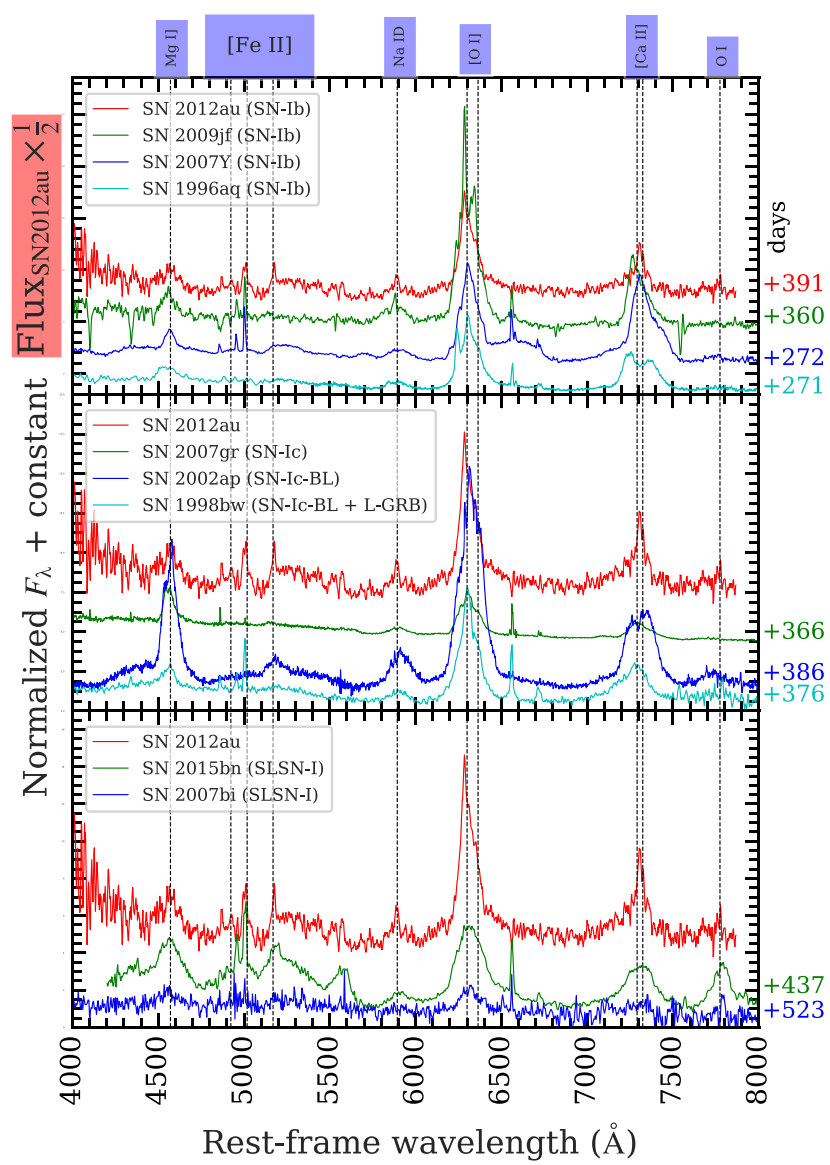

Figure 18. The late nebular spectrum (at $+391 \mathrm{~d}$ ) of SN $2012 \mathrm{au}$ (in red) is compared with some SNe Ib, Ic, and slow-decaying SLSNe I. The comparison spectra of SNe Ib and Ic are chosen at similar phases to SN 2012au, whereas the spectra of SLSNe I are taken at later epochs $(>+400 \mathrm{~d})$.

the $\mathrm{H}$ envelope artificially, the model evolves until the exhaustion of helium; we then impose an artificial $\dot{M} \gtrsim 10^{-4} \mathrm{M}_{\odot} \mathrm{yr}^{-1}$ until the total $\mathrm{H}$ mass of the star decreases to $0.01 \mathrm{M}_{\odot}$. On reaching the specified H-mass limit, we switch off artificial mass loss and the model evolves until the onset of core collapse. The final parameters for all models are listed in Table 3. Fig. 19 shows the STELLA/SNEC results obtained for the models mentioned above. Our models with explosion energy of $15 \times 10^{51} \mathrm{erg}$ and $M_{\mathrm{Ni}}$ in the range $0.2-0.3 \mathrm{M}_{\odot}$ along with CSMI can explain the observed peak luminosity fairly well, but fail to explain the overall light-curve shape beyond the peak light of SN 2012au. These models could satisfactorily explain the photospheric velocities (inferred from Fe II line velocities) as shown in the right panels of Fig. 19. The semi-analytical light curve modelling using MINIM shows the possibility of SN 2012au being powered by a magnetar. Following Metzger et al. (2015), the magnetar spin-down luminosity is given by

$L_{\mathrm{sd}}=L_{\mathrm{sd}_{\mathrm{i}}}\left(1+t / t_{\mathrm{sd}}\right)^{-2}$.

Here, $L_{\mathrm{sd}_{\mathrm{i}}}$ is the spin-down luminosity at $t=0$, and $t_{\mathrm{sd}}$ is the initial spin-down time. This is the luminosity that we inject into the whole ejecta above the mass cut uniformly in mass. The explosion using the magnetar model is simulated using another publicly available code SNEC (Morozova et al. 2015) by following the methods of Aryan et al. (2021). For the initial spin-down luminosity, we assume $L_{\mathrm{sd}_{\mathrm{i}}}=2.2 \times 10^{43} \mathrm{erg} \mathrm{s}^{-1}$, while for the initial spin-down time we 
Table 3. MESA model explosion parameters.

\begin{tabular}{|c|c|c|c|c|c|c|c|c|c|c|c|c|}
\hline Model name & $\begin{array}{c}M_{\text {ZAMS }} \\
\left(\mathrm{M}_{\odot}\right)\end{array}$ & $Z$ & $\left(v / v_{c}\right)$ ZAMS $^{a}$ & $f_{\mathrm{ov}}^{b}$ & $\begin{array}{l}M_{\mathrm{f}}{ }^{c} \\
\left(\mathrm{M}_{\odot}\right)\end{array}$ & $\begin{array}{l}M_{\mathrm{c}}{ }^{d} \\
\left(\mathrm{M}_{\odot}\right)\end{array}$ & $\begin{array}{c}M_{\mathrm{ej}} \\
\left(\mathrm{M}_{\odot}\right)\end{array}$ & $\begin{array}{c}M_{\mathrm{Ni}} \\
\left(\mathrm{M}_{\odot}\right)\end{array}$ & $\begin{array}{c}E_{\exp }^{e} \\
\left(10^{51} \mathrm{erg}\right)\end{array}$ & $\begin{array}{c}t_{\mathrm{CSM}}{ }^{f} \\
(\mathrm{y})\end{array}$ & $\begin{array}{c}\dot{M} \mathrm{y}^{-1} \\
\left(\mathrm{M}_{\odot} \mathrm{y}^{-1}\right)\end{array}$ & $\begin{array}{c}v_{\mathrm{CSM}^{g}}{ }^{g} \\
\left(\mathrm{~km} \mathrm{~s}^{-1}\right)\end{array}$ \\
\hline M20_Z0.02 & 20 & 0.02 & 0.0 & 0.01 & 6.74 & 1.78 & 4.96 & 0.23 & 15 & 0.0 & 0.0 & 0.0 \\
\hline M20_Z0.04 & 20 & 0.04 & 0.0 & 0.01 & 7.07 & 2.11 & 4.96 & 0.23 & 15 & 0.0 & 0.0 & 0.0 \\
\hline M20_Z0.04_CSM & 20 & 0.04 & 0.0 & 0.01 & 6.74 & 1.78 & 4.96 & 0.23 & 15 & 1.3 & 0.0001 & 12.0 \\
\hline M20_Z0.04 MAGNETAR & 20 & 0.04 & 0.0 & 0.01 & 6.74 & 1.44 & 5.30 & 0.00 & 5.0 & 0.0 & 0.0 & 0.0 \\
\hline M22ZZ0.02 & 22 & 0.02 & 0.0 & 0.01 & 7.35 & 2.35 & 5.00 & 0.21 & 15 & 0.0 & 0.0 & 0.0 \\
\hline M22_Z0.04 & 22 & 0.04 & 0.0 & 0.01 & 7.71 & 1.64 & 6.07 & 0.20 & 15 & 0.0 & 0.0 & 0.0 \\
\hline M25_Z0.02 & 25 & 0.02 & 0.0 & 0.01 & 8.50 & 1.97 & 6.53 & 0.21 & 15 & 0.0 & 0.0 & 0.0 \\
\hline M25_Z0.03 & 25 & 0.03 & 0.0 & 0.01 & 8.63 & 1.99 & 6.64 & 0.25 & 15 & 0.0 & 0.0 & 0.0 \\
\hline M25ZZ0.04 & 25 & 0.04 & 0.0 & 0.01 & 9.35 & 1.85 & 7.5 & 0.20 & 15 & 0.0 & 0.0 & 0.0 \\
\hline
\end{tabular}

Note. ${ }^{a}$ initial rotation, ${ }^{b}$ overshooting parameter, ${ }^{c}$ final mass, ${ }^{d}$ mass of the central remnant, ${ }^{e}$ explosion energy. Parameters controlling the extent of CSMI: ${ }^{f}$ wind duration, ${ }^{g}$ CSM velocity.

assume $t_{\mathrm{sd}}=25 \mathrm{~d}$ (close to the value obtained from MINIM). The effects of Ni heating are ignored in this model. Following Metzger et al. (2015) (their equations 2 and 3), corresponding to $L_{\mathrm{sd}_{\mathrm{i}}}=$ $2.2 \times 10^{43} \mathrm{erg} \mathrm{s}^{-1}$ and $t_{\mathrm{sd}}=25 \mathrm{~d}$, we obtain $B \sim 2.0 \times 10^{14} \mathrm{G}$ and $P_{\mathrm{i}} \sim 23 \mathrm{~ms}$ for the modelled magnetar. These values of $B$ and $P_{\mathrm{i}}$ are close to those obtained using MINIM. We see that a $20-\mathrm{M}_{\odot}$ ZAMS progenitor with $Z=0.04$ could explain the observed bolometric luminosity and photospheric velocities nicely, assuming the magnetar powering mechanism. Thus, the inclusion of the magnetar powering mechanism greatly improves light-curve matching even in late phases, as shown in the inset plot of the leftmost bottom panel of Fig. 19.

\section{DISCUSSION AND RESULTS}

In this work, we present well-calibrated optical photometric $(-0.2$ to $+413 \mathrm{~d})$, polarimetric $(-2$ to $+31 \mathrm{~d})$ and optical $(-5$ to +391 d), NIR ( -5 to +22 d) spectroscopic studies of SN 2012au, based on data obtained using many observational facilities around the globe. Analysis based on our photometric observations suggests that SN 2012au appears to be one of the most luminous $\mathrm{SNe} \mathrm{Ib}$ $\left(M_{B \text {, peak }}=-18.06 \pm 0.12 \mathrm{mag}\right)$, though fainter than the threshold limit of SLSNe I $\left(M_{\mathrm{g}}<-19.8 \mathrm{mag}\right.$; Quimby et al. 2018). The $M_{R \text {, peak }}(\sim-18.67 \pm 0.11 \mathrm{mag})$ of SN 2012au is brighter than the average values of $\mathrm{SNe} \mathrm{Ib}$ and Ic, but closer to those reported for $\mathrm{SNe}$ Ic-BL (Drout et al. 2011). Similarly, the peak bolometric luminosity of SN 2012au $\left(\sim[6.56 \pm 0.70] \times 10^{42} \mathrm{erg} \mathrm{s}^{-1}\right)$ is higher than the mean peak luminosities of $\mathrm{SNe} \mathrm{Ib}$ and Ic, but still lower than those of SNe Ic-BL (Lyman et al. 2016). Using the early bolometric light curve of SN 2012au, the estimated values of $M_{\mathrm{ej}}, E_{k}, M_{\mathrm{Ni}}$, and $T_{0}$ are $\sim 5.1 \pm 0.7 \mathrm{M}_{\odot}, \sim(4.8 \pm 0.6) \times 10^{51} \mathrm{erg}, \sim 0.27-0.30 \mathrm{M}_{\odot}$, and $\sim 66.0 \pm 9.4 \mathrm{~d}$, respectively. These physical parameters of SN 2012au are close to those inferred for SN 2009jf (a bright SN Ib: Sahu et al. 2011) and - on average - larger than for classical SNe Ib/c but smaller than for some SNe Ic-BL. SN 2012au manifests larger $M_{\mathrm{ej}}$ and $M_{\mathrm{Ni}}$ in comparison with most of the SNe IIb, Ib, and Ic, which may be the prime reason behind the luminous peak of SN 2012au, as seen in the case of SLSNe I (Nicholl et al. 2015). On the other hand, light-curve decline rates of SN 2012au (at phases $\geq+40 \mathrm{~d}$ ) in all the optical bands are shallower than typically observed in the case of $\mathrm{SNe} \mathrm{Ib}$ and slowdecaying SLSNe I, and theoretically predicated for ${ }^{56} \mathrm{Co} \rightarrow{ }^{56} \mathrm{fe}$ decay. As SN 2012au exhibits comparatively larger $M_{\mathrm{ej}}$, a larger optical depth resulting in a larger diffusion time-scale (for the trapped energy to cross the outer envelope) could broaden the light curve.
Therefore, high trapping of gamma-rays at late phases or higher opacity of massive ejecta are among the plausible interpretations for the modest luminosity decline rate of SN 2012au in comparison with other SNe Ib (Clocchiatti \& Wheeler 1997). However, smoothly distributed circumstellar media up to a larger radius could be another possibility behind the late-time shallower decay rate for SN 2012au, but an absence of the CSMI in the late-time spectra ruled out this scenario (Milisavljevic et al. 2018). The late-time bolometric light curve of SN 2012au is better constrained by $L \propto t^{-2}$, a conventional magnetic dipole equation. Hence for SN 2012au the shallower decay of the late-time light curve might be a potential indicator of a central engine powering source that is accelerating the inner ejecta.

The analytical light-curve modelling of SN 2012au using the MINIM code infers that a spin-down millisecond magnetar plausibly powers the observed luminosity of SN 2012au. This outcome is also consistent with the late-time light-curve reproduced by the equation of a magnetic dipole. Some of the physical parameters obtained using the MINIM/MAG model are $M_{\mathrm{ej}} \approx 4.72 \pm 1.03 \mathrm{M}_{\odot}, v_{\mathrm{exp}} \sim$ $(11.66 \pm 0.58) \times 10^{3} \mathrm{~km} \mathrm{~s}^{-1}, B \sim(8.05 \pm 0.15) \times 10^{14} \mathrm{G}$, and $P_{\mathrm{i}} \sim 18.26 \pm 0.01 \mathrm{~ms}$. The MAG model gives reasonable values of $M_{\mathrm{ej}}$ and $v_{\mathrm{exp}}$, closer to those obtained from the photometric and spectral analysis. For the magnetar powering source of SN 2012au, $B$ is closer and $P_{\mathrm{i}}$ is higher than observed in the case of Type $\mathrm{Ib}$ SN 2005bf (Maeda et al. 2007). On the other hand, for SN 2012au $P_{i}$ is higher and $B$ lies at the top of the range of $P_{i}$ and $B$ values ( $\sim 1-8 \mathrm{~ms}$ and $\sim 1-8 \times 10^{14} \mathrm{G}$, respectively) compared with those observed for SLSNe I (see fig. 10 of Kumar et al. 2021).

Following the results obtained using semi-analytical light curve modelling code MINIM, a magnetar powering mechanism is built through MESA and SNEC. After implementing the magnetar powering mechanism, a $20-\mathrm{M}_{\odot}$ ZAMS progenitor with $Z=0.04$ could reproduce the observed bolometric luminosity and $v_{\mathrm{ph}}$ reasonably. The ${ }^{56} \mathrm{Ni} \rightarrow{ }^{56} \mathrm{Co}$ decay and CSM interaction powering mechanisms using MESA and STELLA, adopting an explosion energy of $15 \times 10^{51}$ erg and $M_{\mathrm{Ni}}$ of $\sim 0.2 \mathrm{M}_{\odot}$, produced a rather poorer match to the observed bolometric light curve. We could see that the inclusion of the magnetar powering mechanism could significantly improve the fit even in the later phases $(+300$ to $+400 \mathrm{~d})$. These results enhance the probability of the SN 2012au explosion being powered by a magnetar.

Our imaging polarimetric data of SN 2012au show signatures of asphericity in the ejecta. The observed polarization values of SN 2012au are significantly higher than those of the field stars lying within a $10^{\circ}$ radius of the SN location. Similar to SN 2008D, we 

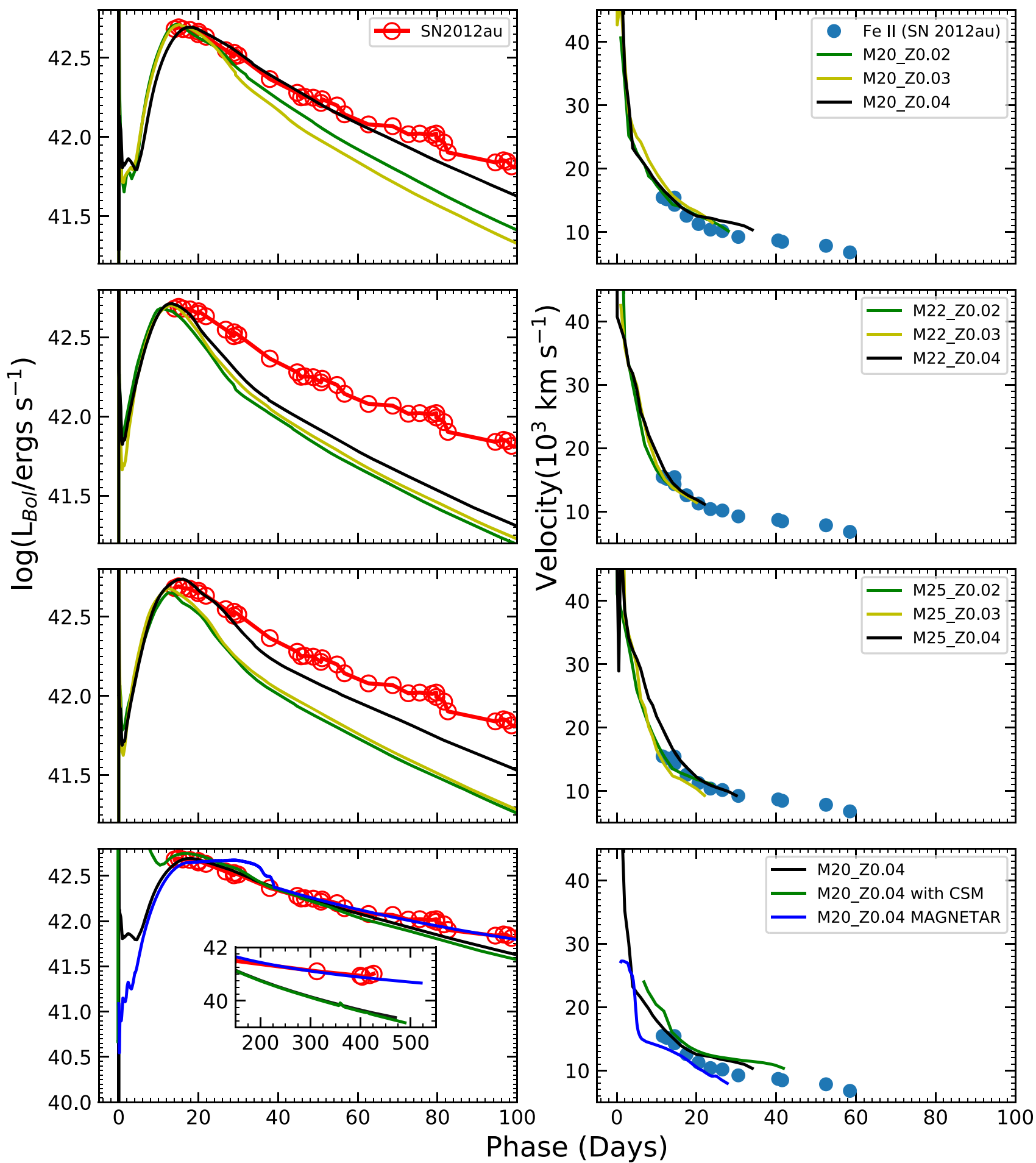

Figure 19. The panels in the left column represent various light curves obtained with progenitor masses $20 \mathrm{M}_{\odot}$ (top panel), $22 \mathrm{M}_{\odot}$ (second panel from top), and $25 \mathrm{M}_{\odot}$ (third panel from top), respectively, and with different metalicities. The leftmost bottom panel shows the $20-\mathrm{M}_{\odot}$ model with $Z=0.04$ that best explains the observed light curve. All these models are generated using STELLA, except the case where SNEC is used to employ the magnetar powering mechanism. The inset plot in the bottom light-curve plot shows the improvement achieved due to inclusion of the magnetar model over other models. The panels in the right column compare the Fe II line velocities of our models with those obtained through the absorption troughs of Fe II lines in the observed spectra.

observe a variation in the polarization parameters of SN 2012au. Near the peak, SN 2012au resembles a percent $P$ value closer to those of SN 2006aj and SN 2007uy. Among Type Ib SNe, SN 2012au presents higher per cent $P, M_{\mathrm{Ni}}$, and $E_{k} / M_{\mathrm{ej}}$ values in comparison with
SN 2008D and iPTF13bvn, but closer to those observed in the case of SN 2007uy.

Spectroscopic observations of SN 2012au reveal that it shares spectral features with a typical $\mathrm{SNe} \mathrm{Ib}$. The absence of the prominent 
W-shaped O II features in the near-peak spectra of SN 2012au confirm its spectral divergence from SLSNe I (Quimby et al. 2011, 2018). The emergence of $\mathrm{O}$ II features generally requires a photospheric temperature of $\gtrsim 12000 \mathrm{~K}$, which could be obtained very easily in the hot ejecta of SLSNe I. On the other hand, near the peak, SN 2012au presents a photospheric temperature of $\approx 9000 \mathrm{~K}$ for the near-peak spectra. In the hot photospheric phase, SN 2012au, like other classical SNe Ib, exhibits prominent He I $(\lambda \lambda 5876,6678$, and $\lambda 7065)$ features from the start of our spectral observations (from $-5 \mathrm{~d}$ ). Near the maximum light of SN 2012au, expansion velocities of He I and Ca II NIR are $\sim 14500 \mathrm{~km} \mathrm{~s}^{-1}$, whereas Fe II displays slightly lower velocity $\left(\sim 12500 \mathrm{~km} \mathrm{~s}^{-1}\right)$, while these line velocities are higher than those of typical SNe Ib. The photospheric velocity (Fe II ion velocity) of SN 2012au decays faster than that of SLSNe I (Nicholl et al. 2015). Using the $v_{\mathrm{ph}}$ obtained from the SYNAPPS spectral fitting to the near-peak spectrum of SN 2012au, we constrained values of $r_{\mathrm{ph}}$ $\sim 1.8 \times 10^{15} \mathrm{~cm}, \tau_{\text {total }} \sim 63.8, M_{\mathrm{ej}} \sim 8.3 \mathrm{M}_{\odot}$, and $E_{k} \sim 5.4 \times 10^{51}$ erg.

The [O I] $\lambda \lambda 6300,6363$ doublet and [Ca II] $\lambda \lambda 7291,7324$ emerge later (from $+90 \mathrm{~d}$ ) in the nebular phase compared with O I $\lambda 7774$, Na I $\mathrm{D}, \mathrm{Mg} \mathrm{I}], \mathrm{Ca}$ II H\&K , and NIR, and the Fe II triplet features can be seen from $+60 \mathrm{~d}$. In the spectra at $+275,+323$, and $+391 \mathrm{~d}$, profiles of $\mathrm{Mg} \mathrm{I}]$, the [O I] doublet, and [Ca II] features are asymmetric, which is indicative of synthesized elements being distributed asymmetrically or mixing and clumps in the ejecta of SN 2012au. The plausible reasons behind the asymmetric blueshifted [OI] line profile as a narrow peak on a broader base are large-scale clumping, a unipolar jet, or a single massive blob moving towards the observer. These findings about asymmetry are in conformity with those obtained using the imaging polarimetric observations discussed above. The nebular spectrum of SN 2012au at $+391 \mathrm{~d}$ is closely matched with the modelled spectrum produced for a progenitor with $M_{\text {ZAMS }}$ of $17 \mathrm{M}_{\odot}$ by Jerkstrand et al. (2015), considering strong mixing and dust in the ejecta. From the spectrum at +323 d of SN 2012au and equation (2) of Jerkstrand et al. (2014), we infer $F$ ([O I] $\lambda 5577$ ) $\approx(1.18 \pm 0.56) \times 10^{-14}$ and $F([\mathrm{O} \mathrm{I}]$ doublet $) \approx(1.12 \pm 0.07) \times 10^{-13}$ $\mathrm{erg} \mathrm{s}^{-1} \mathrm{~cm}^{-2}$, which confers O I temperature $\approx 4098.39 \pm 309.15 \mathrm{~K}$ and $M_{\mathrm{O}} \approx 1.62 \pm 0.15 \mathrm{M}_{\odot}$. The $M_{\mathrm{O}}$ of SN 2012au is higher than the one observed for SN 2009jf (1.34 $\mathrm{M}_{\odot}$ : Sahu et al. 2011) and also higher than the $M_{\mathrm{O}}$ range $\left(0.1-1.4 \mathrm{M}_{\odot}\right)$ estimated for a sample of SESNe by Elmhamdi et al. (2004). Using this $M_{\mathrm{O}}$, we also constrained values of $M_{\text {ZAMS }}\left(\sim 20-25 \mathrm{M}_{\odot}\right)$ and $M_{\mathrm{He}}\left(\sim 4-8 \mathrm{M}_{\odot}\right)$. The $M_{\text {ZAMS }}$ of SN 2012au is higher than those for well-studied SNe Ib (except SN 2009jf) but lower than for the SLSNe I tabulated in Table 1 .

In nebular spectra of SN 2012au, there are no signatures of CSMI up to +391 d. Additionally, Milisavljevic et al. (2018) also did not notice any signatures of CSMI in a very late-time (at $+2270 \mathrm{~d}$ ) spectrum of SN 2012au. On the other hand, a pulsar wind nebula as a heating source that could be generated by the spin-down power of a central pulsar was proposed by Milisavljevic et al. (2018), which is in agreement with the results from analytical light-curve modelling as described above. Based on the [Ca II]/[O I] ratio of SN 2012au and the findings of Kuncarayakti et al. (2015), we suggest that the progenitor of SN 2012au is a single WR star with $M_{\text {ZAMS }} \approx 25 \mathrm{M}_{\odot}$. SN 2012au displays a higher $\mathrm{Mg} \mathrm{I}] /[\mathrm{O} \mathrm{I}]$ flux ratio in comparison with typical Type Ib/c events, though comparable to some of the well-studied SLSNe I. A higher value of $\mathrm{Mg}$ I]/[O I] ratio of SN 2012au up to $+323 \mathrm{~d}$ indicates a higher degree of outer envelope stripping (Foley et al. 2003). From +320 to $+390 \mathrm{~d}$, the $\mathrm{Mg}$ I] and [O I] flux ratio of SN 2012au decreases sharply to $\sim 0.27$, which indicates a lack of high-density enhancements due to clumping, mixing, and asymmetry in the ejecta (Jerkstrand et al. 2015). However, dust formation and blending of the $\mathrm{Mg}$ I] from Fe II ions may be other plausible reasons.

In addition to optical spectral observations, our first NIR spectrum at $-5 \mathrm{~d}$ appears featureless with a few lines of $\mathrm{He} \mathrm{I} \lambda 10800$ (the most prominent one), $\mathrm{CI}, \mathrm{Mg}$ I, O I, and Na I. The strength of absorption features reduces over time and is superseded by other prominent emission lines. The late-time spectra up to $+319 \mathrm{~d}$ exhibit prominent features of the hydrogen Paschen series, HI, He I, Na I, O I, Mg I, $\mathrm{Mg}$ II, Si I, S I, CaI, and Fe II. The first overtone of CO between $\sim 22900$ and $24000 \AA$ is absent in the NIR spectra of SN 2012au (up to $+319 \mathrm{~d}$ ). The $\mathrm{CO}$ emission was also absent in the $+79 \mathrm{~d}$ spectrum of iPTF13bvn (Fremling et al. 2016). The absence of CO molecule formation in the case of SN 2012au could be because of the temperature being higher than the molecule formation threshold, higher mixing of ionized helium between ejecta layers, or a higher $v_{\text {ph }}$ of SN 2012au. The absence of CO emission in the spectra of SN 2012au up to +319 d confirms the lack of emission signatures by heated dust.

In both hot and cool photospheric phases, SN 2012au shares an overall spectral similarity with SN 2015ap, though absorption features in the spectra SN 2012au have a higher blueshift than in the case of SN 2009jf and SN 2015ap, which is in agreement with the comparatively higher $v_{\text {ph }}$ of SN 2012au. In the nebular phase, some of the spectral features, in particular, the [OI] and the [Ca II] doublets, evolve at later times than those observed in other $\mathrm{SNe} \mathrm{Ib}$. In the late phases $(>+250 \mathrm{~d})$, the [O I] and [Ca II] emission lines of SN 2012au match closely those observed in the case of SN 2007Y. Overall, the spectral comparison performed in the present study reveals that the photometrically slow-decaying SN 2012au also evolves spectroscopically on longer time-scales.

\section{CONCLUSION}

In this study, we present well-calibrated photometric, polarimetric, and spectroscopic studies of SN 2012au spanning from $5 \mathrm{~d}$ before the $B$-band maximum to nearly one year post-maximum. SN 2012au exhibits higher peak luminosity in comparison with typical Type IIb, $\mathrm{Ib}$, and Ic, but lower than those observed for Type Ic-BL and SLSNe I. The peak bolometric luminosity of SN 2012au implies a synthesis of $\sim 0.27-0.30 \mathrm{M}_{\odot}$ of ${ }^{56} \mathrm{Ni}$ during the explosion. For SN 2012au, $M_{\mathrm{ej}}$ values constrained using photometric light-curve analysis $(\sim$ 5.1 $\left.\mathrm{M}_{\odot}\right)$, semi-analytical modelling $\left(\sim 4.7 \mathrm{M}_{\odot}\right)$, and spectral analysis $\left(\sim 8.3 \mathrm{M}_{\odot}\right)$ hinted a range of $M_{\mathrm{ej}} \sim 4.7-8.3 \mathrm{M}_{\odot}$ for SN 2012au. The $M_{\mathrm{ej}}\left(\sim 4.7-8.3 \mathrm{M}_{\odot}\right)$ and $E_{k}\left(\sim[4.8-5.4] \times 10^{51} \mathrm{erg}\right)$ of SN 2012au are also higher in comparison with most Type IIb, Ib, and Ic SNe, but lower than those of Ic-BL/GRB-SNe and SLSNe I. Therefore, based on the peak brightness and inferred physical parameters, SN 2012au appears more like a bridge between normal $\mathrm{Ib} / \mathrm{c}$ and Ic-BL, rather than Ib/c and SLSNe I. Comparatively higher values of $M_{\mathrm{Ni}}$ and $M_{\mathrm{ej}}$ could explain the rather luminous peak of SN 2012au. SN 2012au presents the most shallow post-peak decay rate in comparison with Type Ib and slow-decaying SLSNe I, which indicates high trapping of gamma-rays or higher opacity of the massive ejecta.

Analytical light-curve modelling insinuates a spin-down millisecond magnetar with $B \approx(8.05 \pm 0.15) \times 10^{14} \mathrm{G}$ and $P_{\mathrm{i}} \approx$ $18.26 \pm 0.01 \mathrm{~ms}$ as a likely powering source for SN 2012au. The late-time light curve of SN 2012au is flatter than the theoretical ${ }^{56} \mathrm{Co} \rightarrow{ }^{56} \mathrm{fe}$ decay curve and consistent with the equation of the standard magnetic dipole, also favouring a central engine powering source. Additionally, results from MESA and SNEC support a magnetar powering mechanism with a progenitor having $M_{\text {ZAMS }} \sim 20 \mathrm{M}_{\odot}$ 
and $Z=0.04$. However, ${ }^{56} \mathrm{Ni} \rightarrow{ }^{56} \mathrm{Co}$ decay and CSM interaction powering mechanisms produced a comparatively poorer match in MESA. Based on the absence of narrow $\mathrm{H}$ Balmer lines in the latetime spectrum (at $+2270 \mathrm{~d}$ ), Milisavljevic et al. (2018) exclude the possibility of CSMI as the primary powering source of SN 2012au. In addition, Milisavljevic et al. (2018) propose a pulsar wind nebula as a heating source of SN 2012au that could be generated by the spin-down power of a central pulsar. Also, a central engine powering source is very likely to explain observed asphericity, mixing, and the comparatively higher $v_{\text {ph }}$ of SN 2012au ejecta (Chen, Woosley \& Whalen 2020). These results together strengthen the probability of the SN 2012au explosion being powered by a magnetar.

Imaging polarization values of SN 2012au are significantly higher than those of field stars, favouring asphericity in the ejecta. Also, in the nebular spectra of SN 2012au ( $\geq 270 \mathrm{~d}$ ), asymmetric profiles of $\mathrm{Mg} I$, the $[\mathrm{OI}]$ doublet, and [CaII] features indicate synthesized elements being distributed asymmetrically and/or clumpy ejecta. However, the shape of the [OI] line profile is a narrow peak on a broader base that is blueshifted, and suggests large-scale clumping, a unipolar jet, or a single massive blob moving towards the observer. The sharply decreasing behaviour of the $\mathrm{Mg}$ I]/[OI] flux ratio of SN 2012au from +323 to $+391 \mathrm{~d}$ indicates clumping, mixing, and asymmetry in the ejecta. The first overtone of $\mathrm{CO}$ is absent in the NIR spectra of SN 2012au, indicative of high temperature and strong mixing of ionized helium between ejecta layers, and shows a lack of emission signatures by heated dust. Overall, these observational signatures favour mixing and asymmetry in the ejecta of SN 2012au.

The spectral evolution of SN 2012au is similar to that of other typical SNe Ib. However, spectral comparison reveals that the photometrically slow-decaying SN 2012au also evolves more slowly spectroscopically. The near-peak spectra of SN 2012au show a clear absence of W-shaped O II features, commonly observed in SLSNe I. $\mathrm{SN} 2012 \mathrm{au}$ shows higher $v_{\mathrm{ph}}$ than other $\mathrm{SNe} \mathrm{Ib}$, but evolves faster than SLSNe I. Using the fluxes of the [O I] doublet and [Ca II], we propose that the progenitor of SN 2012au is a single WR star, with $M_{\mathrm{O}}$ and $M_{\mathrm{He}}$ of $\sim 1.62 \pm 0.15 \mathrm{M}_{\odot}$ and $\sim 4-8 \mathrm{M}_{\odot}$, respectively. $M_{\text {ZAMS }}$ values constrained using the $+391 \mathrm{~d}$ spectrum matching those of the modelled spectrum of Jerkstrand et al. $(2015)\left(\sim 17 \mathrm{M}_{\odot}\right)$, from $M_{\mathrm{O}}$ and the $[\mathrm{Ca}$ II $] /[\mathrm{O} \mathrm{I}]$ doublet flux ratio $\left(\sim 25 \mathrm{M}_{\odot}\right)$, and also those constrained using MESA and SNEC modelling $\left(\sim 20 \mathrm{M}_{\odot}\right)$, suggest a range of $M_{\text {ZAMS }} \sim 17-25 \mathrm{M}_{\odot}$ for $\mathrm{SN} 2012 \mathrm{au}$. The above physical parameters are close to those inferred for some SNe Ic-BL (e.g. SN 1998bw and SN 2002ap), but lower than those of SLSNe I (e.g. PTF12dam and SN 2015bn), favouring SN 2012au as a bridge between normal Ib/c and Ic-BL.

\section{ACKNOWLEDGEMENTS}

This study uses data from DOT-3.6m, HCT-2.0m, DFOT$1.3 \mathrm{~m}$, and ST-1.04m, and the authors of this work are highly grateful to the observers at the Aryabhatta Research Institute of Observational Sciences (ARIES) and Indian Astronomical Observatory (IAO), Hanle for their valuable time and support for the observations of this event. This study also uses data from the BTA-6.0m at SAO Russia. Observations with the SAO RAS telescopes are supported by the Ministry of Science and Higher Education of the Russian Federation (including agreement No05.619.21.0016, project ID RFMEFI61919X0016). This article is partially based on observations collected at the Galileo 1.22$\mathrm{m}$ telescope operated by DFA University of Padova (Asiago, Italy). For the present study, the spectroscopic observations have also been taken in the framework of the European supernova collaboration involved in ESO-NTT large program 184.D-1140 led by Stefano Benetti. SBP, RG, AA, and KM acknowledge BRICS grant DST/IMRCD/BRICS/Pilot call/ProFCheap/2017(G) and DST/JSPS grant DST/INT/JSPS/P/281/2018 for this work. BK, GCA, and DKS acknowledge BRICS grant DST/IMRCD/BRICS/PilotCall1/MuMeSTU/2017(G) for the present work. AA also acknowledges funds and assistance provided by the Council of Scientific \& Industrial Research (CSIR), India. JV is supported by the project 'Transient Astrophysical Objects' GINOP 2.3.2-15-2016-00033 of the National Research, Development, and Innovation Office (NKFIH), Hungary, funded by the European Union. Research by SV is supported by NSF grants AST-1813176 and AST-2008108. The authors are thankful to the anonymous referee for constructive comments and suggestions to improve the overall analysis presented in this work. SBP and AK are highly grateful to Professor J. Craig Wheeler for his consistent support and guidance in learning many aspects of the frontiers of CCSNe physics. AK and BK also acknowledge C. Eswaraiah, Kaushal Sharma, and Raya Dastidar for valuable discussions on various aspects and S. Bose for observing the event with the ST 1.04-m. BK thanks M. Yamanaka and K. S. Kawabata for sharing the data. This research has utilized the NED, which is operated by the Jet Propulsion Laboratory, California Institute of Technology, under contract with NASA. We acknowledge the use of NASA's Astrophysics Data System Bibliographic Services. This research also made use of the Open Supernova Catalog (OSC), currently maintained by James Guillochon and Jerod Parrent. The work was partially performed as part of the government contract of the SAO RAS approved by the Ministry of Science and Higher Education of the Russian Federation. Observations at the SAO RAS telescopes are supported by the Ministry of Science and Higher Education of the Russian Federation.

\section{DATA AVAILABILITY}

The data used in this work can be made available on request to the corresponding authors.

\section{REFERENCES}

Arnett W. D., 1982, ApJ, 253, 785

Arnett W. D., 1996, Supernovae and Nucleosynthesis: An Investigation of the History of Matter from the Big Bang to the Present. Princeton Univ. Press, Princeton, $\mathrm{NJ}$

Aryan A. et al., 2021, MNRAS, 505, 2530

Banerjee D. P. K. et al., 2018, MNRAS, 481, 806

Blinnikov S. I. Eastman R. Bartunov O. S. Popolitov V. A. Woosley S. E. 1998, ApJ, 496, 454

Blinnikov S. I. Lundqvist P. Bartunov O. Nomoto K. Iwamoto K. 2000, ApJ, 532,1132

Blinnikov S. I. et al., 2006, A\&A, 453, 229

Branch D., Wheeler J. C., 2017, Supernova Explosions: Astronomy and Astrophysics Library Supernova, Springer, Berlin

Branch D., Jeffery D. J., Young T. R., Baron E., 2006, PASP, 118, 791

Cano Z., 2013, MNRAS, 434, 1098

Cano Z., Wang S. Q., Dai Z. G., Wu X. F., 2017, Advances in Astronomy, 2017E, 5C

Cao Y. et al., 2013, ApJ, 775, L7

Chatzopoulos E., Tuminello R., 2019, ApJ, 874, 68

Chatzopoulos E., Wheeler J. C., Vinko J., 2009, ApJ, 704, 1251

Chatzopoulos E. Wheeler J. C. Vinko J. et al., 2012, ApJ, 746, 121

Chatzopoulos E. Wheeler J. C. Vinko J. Horvath Z. L. Nagy A. 2013, ApJ, 773,76 
Chen K.-J., Woosley S. E., Whalen D. J., 2020, ApJ, 893, 99

Cherchneff I., Dwek E., 2010, ApJ, 713, 1

Cherchneff I., Lilly S., 2008, ApJ, 683, L123

Chevalier R. A., Fransson C., 1994, ApJ, 420, 268

Chugai N. N., 1992, Sov. Astron. Lett., 18, 168

Clocchiatti A., Wheeler J. C., 1997, ApJ, 491, 375

Covino S. et al., 2003, GCN Circ., 2167, 1C

De Cia A. et al., 2018, ApJ, 860, 100

Dessart L., Hillier D. J., Li C., Woosley S., 2012, MNRAS, 424, 2139

Dessart L., Hillier D. J., Woosley S., Livne E., Waldman R., Yoon S. C., Langer N., 2015, MNRAS, 453, 2189

Doroshenko V. T., Efimov Y. S., Shakhovskoi N. M., 1995, Astron. Lett., 21, 513

Drout M. R. et al., 2011, ApJ, 741, 97

Drout M. R. et al., 2016, ApJ, 821, 57

Eldridge J. J., Maund J. R., 2016, MNRAS, 461, L117

Eldridge J. J., Langer N., Tout C. A., 2011, MNRAS, 414, 3501

Elmhamdi A., Danziger I. J., Cappellaro E., Della V. M., Gouiffes C., Phillips M. M., Turatto M., 2004, A\&A, 426, 963

Elmhamdi A., Danziger I. J., Branch D., Leibundgut B., Baron E., Kirshner R. P., 2006, A\&A, 450, 305

Eswaraiah C., Pandey A. K., Maheswar G., Chen W. P., Ojha D. K., Chandola H. C., 2012, MNRAS, 419, 2587

Fang Q., Maeda K., 2018, ApJ, 864, 47

Fang Q., Maeda K., Kuncarayakti H., Sun F., Gal-Yam A., 2019, Nature Astron., 3, 434

Filippenko A. V., 1997, ARA\&A, 35, 309

Filippenko A. V., Matheson T., Ho L. C., 1993, ApJ, 481, L89

Folatelli G., Van Dyk S. D., Kuncarayakti H., 2016, ApJ, 825, 22

Foley R. J. et al., 2003, PASP, 115, 1220

Fransson C., Chevalier R. A., 1989, ApJ, 343, 323

Fremling C. et al., 2014, A\&A, 565, 114

Fremling C., Sollerman J., Taddia F., 2016, A\&A, 593, 68

Fremling C., Sollerman J., Kasliwal M. M., 2018, A\&A, 618, 37

Gaia Collaboration et al., 2018, A\&A, 616, 1

Gal-Yam A., 2012, Sci, 337, 927

Gal-Yam A., 2017, in Alsabti A., Murdin P., eds, Handbook of Supernovae. Springer, Cham, p. 195

Gal-Yam A., 2019, ARA\&A, 57, 305

Gal-Yam A. et al., 2007, ApJ, 656, 372

Gal-Yam A. et al., 2009, Nature, 462, 624

Gangopadhyay A. et al., 2020, MNRAS, 497, 3770G

Gaskell C. M., Cappellaro E., Dinerstein H. L., Garnett D. R., Harkness R. P., Wheeler J. C., 1986, ApJ, 306, L77

Gearhart R. A., Wheeler J. C., Swartz D. A., 1999, ApJ, 510, 944

Georgy C., Meynet G., Walder R., Folini D., Maeder A., 2009, A\&A, 502, 611

Gerardy C. L. Fesen R. A. Höflich P. Wheeler J. C. 2000, AJ, 119, 2968

Gerardy C. L. Fesen R. A. Nomoto K. Maeda K. Hoflich P. Wheeler J. C. 2002, Publ. Astron. Soc. Jpn, 54, 905

Gilkis A., Vink J. S., Eldridge J. J., Tout C. A., 2019, MNRAS, 486, 4451

Gorosabel J. et al., 2006, A\&A, 459, L33

Gorosabel J. et al., 2010, A\&A, 522, 14G

Green G. M., Schlafly E., Zucker C., Speagle J. S., Finkbeiner D., 2019, ApJ, 887, 93

Groh J. H., Maynet G., Georgy C., Ekstrom S., 2013, A\&A, 558, A13

Gutiérrez C. P. et al., 2021, MNRAS, 504, 4907

Hachinger S., Mazzali P. A., Taubenberger S., Hillebrandt W., Nomoto K., Sauer D. N., 2012, MNRAS, 422, 70

Heger A., Fryer C. L., Woosley S. E., Langer N., Hartmann D. H., 2003, ApJ, 591,288

Henyey L., Vardya M. S., Bodenheimer P., 1965, ApJ, 142, 841

Herwig F., 2000, A\&A, 360, 952

Hoffman J. L. et al., 2014, in American Astronomical Society Meeting Abstracts \#223. p. 354.21

Hoffman J. L., Leonard D. C., Chornock R., Filippenko A. V., Barth A. J., Matheson T., 2008, ApJ, 688, 1186
Hoffman J. L. et al., 2017, in Eldridge J. J., Bray J. C., McClelland L. A. S., Xiao L., eds, The Lives and Death-Throes of Massive Stars, Vol. 329, p. 54

Hoflich P., 1991, A\&A, 246, 481

Höflich P., Khokhlov A., Wang L., 2001, American Institute of Physics Conference Series, 586, $459 \mathrm{H}$

Hogg D. W., Baldry I. K., Blanton M. R., Eisenstein D. J., 2002, preprint (astro-ph/0210394)

Hunter D. J. et al., 2009, A\&A, 508, 371

Inserra C. et al., 2013, ApJ, 770, 128

Inserra C., 2019, Nat. Astron., 3, 697

Jerkstrand A. et al., 2014, MNRAS, 439, 3694

Jerkstrand A., Ergon M., Smartt S. J., Fransson C., Sollerman J., Taubenberger S., Bersten M., Spyromilio J., 2015, A\&A, 573, 12

Jordi K., Grebel E. K., Ammon K., 2006, A\&A, 460, 339

Kamble A. et al., 2013, preprint (arXiv:1309.3573)

Kamble A. et al., 2014, ApJ, 797, 2

Kasen D., Bildsten L., 2010, ApJ, 717, 245

Kawabata K. S. et al., 2003, ApJ, 593, L19

Kilpatrick C. D. et al., 2018, MNRAS, 480, 2072

Kilpatrick C. D. et al., 2021, MNRAS, 504, 2073

Kippenhahn R., Ruschenplatt G., Thomas H.-C., 1980, A\&A, 91, 175

Könyves-Tóth R., Vinko J., 2020, ApJ, 900, 73

Kumar B. et al., 2019, MNRAS, 488, 3089

Kumar B., Pandey S. B., Eswaraiah C., Gorosabel J., 2014, MNRAS, 442, 2

Kumar B., Pandey S. B., Eswaraiah C., Kawabata K. S., 2016, MNRAS, 456, 3157

Kumar B. et al., 2018, Bulletin de la Societe Royale des Sciences de Liege, 87,29

Kumar A. et al., 2020, ApJ, 892, 28K

Kumar A. et al., 2021, MNRAS, 502, 1678

Kuncarayakti H. et al., 2015, A\&A, 579, A95

Langer N., El Eid M. F., Fricke K. J., 1985, A\&A, 145, 179

Leibundgut B., Kirshner R. P., Pinto P. A., Rupen M. P., Smith R. C., Gunn J. E., Schneider D. P., 1991, ApJ, 372, 531

Leloudas G. et al., 2017, ApJ, 837, L14

Leonard D. C., Filippenko A. V., 2005, in Turatto M., Benetti S., Zampieri L., Shea W., eds, ASP Conf. Ser. Vol. 342. Astron. Soc. Pac., San Francisco, p. 330

Leonard D. C., Filippenko A. V., Barth A. J., Matheson T., 2000, ApJ, 536, 239

Leonard D. C. et al., 2006, Nature, 440, 505

Liljegren S., Jerkstrand A., Grumer J., 2020, A\&A, 642, A135

Lyman J. D., Bersier D., James P. A., Mazzali P. A., Eldridge J. J., Fraser M., Pian E., 2016, MNRAS, 457, 328

Maeda K., Nakamura T., Nomoto K., Mazzali P. A., Patat F., Hachisu I., 2002, ApJ, 565, 405

Maeda K., Nomoto K., Mazzali P. A., Deng J., 2006, ApJ, 640, 854

Maeda K. et al., 2007, ApJ, 666, 1069

Maeda K. et al., 2008, Science, 319, 1220

Mauerhan J. et al., 2014, MNRAS, 442, 1166

Mauerhan J. C. et al., 2017, ApJ, 834, 118

Maund J. R., Wheeler J. C., Patat F., Baade D., Wang L., Höflich P., 2007, MNRAS, 381, 201

Maund J. R., Wheeler J. C., Baade D., Patat F., Höflich P., Wang L., Clocchiatti A., 2009, ApJ, 705, 1139

Maund J. R., Steele I., Jermak H., Wheeler J. C. Wiersema K. 2019, MNRAS, 482, 4057

Maurer I., Mazzali P. A., Taubenberger S., Hachinger S., 2010, MNRAS, 409, $1441 \mathrm{M}$

Mazzali P. A. et al., 2002, ApJ, 572, L61

Mazzali P. A., Nomoto K., Patat F., Maeda K., 2001, ApJ, 559, 1047

Mazzali P. A. et al., 2005, Science, 308, 1284

McCall M. L., 1984, MNRAS, 210, 829

Medhi B. J., Maheswar G., Brijesh K., Pandey J. C., Kumar T. S., Sagar R., 2007, MNRAS, 378, 881

Meikle W. P. S. et al., 1996, 281, 263

Metzger B. D., Margalit B., Kasen D., Quataert E., 2015, MNRAS, 454, 3311 
Milisavljevic D. et al., 2013, ApJ, 770, 38

Milisavljevic D., Patnaude D. J., Chevalier R. A., Raymond J. C., Fesen R. A., Margutti R., Conner B., Banovetz J., 2018, ApJ, 864, 36

Modjaz M. et al., 2014, AJ, 147, 99

Modjaz M., Gutiérrez C. P., Arcavi I., 2019, Nature Astron., 3, 717

Mohan V., Uddin W., Sagar R., Gupta S. K., 1999, Bull. Astron. Soc. India, 27, 601

Morgan H. L., Edmunds M. G., 2003, MNRAS, 343, 427

Moriya T. J., Sorokina E. I., Chevalier R. A., 2018, Space Sci. Rev., 214, 59

Morozova V., Piro A. L., Renzo M., Ott C. D., 2015, ApJ, 814, 63

Nadyozhin D. K., 1994, ApJS, 92, 527

Nicholl M., 2018a, Research Notes of the American Astronomical Society, 2,230

Nicholl M. et al., 2013, Nature, 502, 346

Nicholl M. et al., 2015, MNRAS, 452, 3869

Nicholl M. et al., 2016, ApJ, 826, 39

Nicholl M., Guillochon J., Berger E., 2017, ApJ, 850, 55N

Nicholl M. et al., 2018b, ApJ, 866, L24

Nicholl M., Berger E., Blanchard P. K., Gomez S., Chornock R., 2019, ApJ, 871,102

Nomoto K. I., Iwamoto K., Suzuki T., 1995, Phys. Rep., 256, 173

Nomoto K., Tominaga N., Umeda H., Kobayashi C., Maeda K., 2006, Nuclear Physics A, 777, 424

Nugis T., Lamers H. J. G. L. M., 2000, A\&A, 360, 227

Osterbrock D. E., 1989, Sky \& Telescope, 78, 491

Ouchi R., Maeda K., 2017, ApJ, 840, 90

Pandey S. B., Anupama G. C., Sagar R., Bhattacharya D., Sahu D. K., Pandey J. C., 2003, MNRAS, 340, 375P

Pandey J. C., Medhi B. J., Sagar R., Pandey A. K., 2009, MNRAS, 396, 1004

Pandey S. B., Yadav R. K. S., Nanjappa N, Yadav S., Reddy B. K., Sahu S., Srinivasan R., 2018, Bull. Soc. R. Sci. Liege, 87, 42

Pastorello A. et al., 2010, ApJ, 724, L16

Patat F. et al., 2001, ApJ, 555, 900

Paxton B., Bildsten L., Dotter A., Herwig F., Lesaffre P., Timmes F., 2011, ApJS, 192, 3

Paxton B. et al., 2013, ApJS, 208, 4

Paxton B. et al., 2015, ApJS, 220, 15

Paxton B. et al., 2018, ApJS, 234, 34

Pettini M., Pagel B. E. J., 2004, MNRAS, 348, L59

Podsiadlowski P., Joss P. C., Hsu J. J. L., 1992, ApJ, 391, 246

Prentice S. J., Mazzali P. A., 2017, MNRAS, 469, 2672

Prentice S. J. et al., 2019, MNRAS, 485, 1559

Quimby R. M. et al., 2011, Nature, 474, 487

Quimby R. M. et al., 2018, ApJ, 855, 2

Ramaprakash A. N., Gupta R., Sen A. K., Tandon S. N., A\&AS, 128, 369

Rautela B. S., Joshi G. C., Pandey J. C., 2004, Bull. Astron. Soc. India, 32, 159

Reilly E. et al., 2016, MNRAS, 457, 288R

Rho J. et al., 2021, ApJ, 908, 232

Sahu D. K., Gurugubelli U. K., Anupama G. C., Nomoto K., 2011, MNRAS, 413,2583

Sahu D. K., Anupama G. C., Chakradhari N. K., Srivastav S., Tanaka M., Maeda K., Nomoto K., 2018, MNRAS, 475, 2591S

Sanders N. E. et al., 2012, ApJ, 758, 132

Sarangi A., Cherchneff I., 2013, ApJ, 776, 107

Sarangi A., Dwek E., Arendt R. G., 2018, ApJ, 859, 66

Schlafly E. F., Finkbeiner D. P., 2011, ApJ, 737, 103

Schmidt G. D., Elston R., Lupie O. L., 1992, AJ, 104, 1563

Serkowski K., Mathewson D. S., Ford V. L., 1975, ApJ, 196, 261

Shapiro P. R., Sutherland P. G., 1982, ApJ, 263, 902

Shivvers I. et al., 2017, PASP, 129, 054201

Shivvers I. et al., 2019, MNRAS, 482, 1545

Smartt S. J., 2009, ARA\&A, 47, 63

Smith N., Gehrz R. D., Campbell R., Kassis M., Le M. D, Kuluhiwa K., Filippenko A. V., 2011, MNRAS, 418, 1959
Spyromilio J., Leibundgut B., Gilmozzi R., 2001, A\&A, 376, 188

Srivastav S., Anupama G. C., Sahu D. K., 2014, MNRAS, 445, 1932

Srivastav S., Ninan J. P., Kumar B., Anupama G. C., Sahu D. K., Ojha D. K., Prabhu T. P., 2016, MNRAS, 457, 1000

Stalin C. S., Hegde M., Sahu D. K., Parihar P. S,, Anupama G. C., Bhatt B. C., Prabhu T. P., 2008, Bull. Astron. Soc. India, 36, 111

Stevance H. F., 2019, preprint (arXiv:1906.07184)

Stevance H. F. et al., 2017, MNRAS, 469, 1897S

Stritzinger M. et al., 2009, ApJ, 696, 713

Stritzinger M. D. et al., 2020, A\&A, 634, A21

Taddia F. et al., 2015, A\&A, 574, 60

Taddia F. et al., 2018, A\&A, 609, 136

Takaki K. et al., 2013, ApJ, 772, 17

Tanaka M., 2017, Phil. Trans. R. Soc. London Ser. A, 375, 20160273

Tanaka M., Kawabata K. S., Maeda K., Hattori T., Nomoto K., 2008, ApJ, 689,1191

Taubenberger S. et al., 2009, MNRAS, 397, 677

Thielemann F.-K., Nomoto K., Hashimoto M.-A., 1996, ApJ, 460, 408

Thomas R. C., Nugent P. E., Meza J. C., 2011, PASP, 123, 237

Uomoto A., 1986, ApJ, 310, L35

Valenti S. et al., 2008, MNRAS, 383, 1485

Valenti S. et al., 2011, MNRAS, 416, 3138

Van Dyk S. D. et al., 2018, ApJ, 860, 90

Van Dyk S. D., 2017, Phil. Trans. R. Soc. London Ser. A, 375, 20160277

van Leeuwen F., 2007, A\&A, 474, 653

Vink J. S., de Koter A., Lamers H. J. G. L. M., 2001, A\&A, 369, 574

Wang L., Wheeler J. C., 2008, ARA\&A, 46, 433

Wang L., Zhou H., 2003, ApJ, 592, 457

Wang S.-Q., Wang L.-J., Dai Z.-G., 2019, Res. Astron. Astrophys., 19, 063

Wheeler J. C., Harkness R. P., Barker E. S., Cochran A. L., Wills D., 1987, ApJ, 313, L69

Wheeler J. C., Hoeflich P., Harkness R. P., Spyromilio J., 1998, ApJ, 496, 908

Wheeler J. C., Johnson V., Clocchiatti A., 2015, MNRAS, 450, 1295

Wheeler J. C., Chatzopoulos E., Vinkó J., Tuminello R., 2017, ApJ, 851, L14

Woosley S. E., 2010, ApJ, 719, L204

Xiang D. et al., 2019, ApJ, 871, 176

Yoon S.-C., 2015, PASA, 32, E015

Yoon S.-C., Woosley S. E., Langer N., 2010, ApJ, 725, 940

\section{SUPPORTING INFORMATION}

Supplementary data are available at MNRAS online.

Table S1. Calibrated magnitudes (DOT-3.6m and HCT-2m) of the secondary standard stars in the SN 2012au field, as shown in Fig. 1. Table S2. Photometric data of SN 2012au in $U, B, V, R$, and $I$ bands along with one-epoch observations in $J, H$, and $K$ bands.

Table S3. Log of polarimetric observations using the AIMPOL instrument and estimated parameters of SN 2012au during early phases.

Table S4. Observational details of nine isolated field stars selected to subtract the interstellar polarization.

Table S5. Optical and NIR spectroscopic data of SN 2012au obtained using Galileo-1.22m, HCT-2m, CAHA-2.2m, NTT-3.58m, and BTA$6 \mathrm{~m}$.

Please note: Oxford University Press is not responsible for the content or functionality of any supporting materials supplied by the authors. Any queries (other than missing material) should be directed to the corresponding author for the article.

This paper has been typeset from a $\mathrm{T}_{\mathrm{E}} \mathrm{X} / \mathrm{ET} \mathrm{E} \mathrm{X}$ file prepared by the author. 